\title{
Voltage-gating and assembly of split Kv10.1 channels
}

\author{
Dissertation
}

In partial fulfilment of the requirements

for the degree "Doktor rerum naturalium" (Dr. rer. nat.)

in the Neuroscience Program

at the Georg August Universität Göttingen,

Faculty of Biology

\section{Submitted by}

Adam Tomczak

Born in

Poznan, Poland

Göttingen, 2016 


\section{Members of the Thesis Committee:}

Prof. Dr. Luis Pardo, Reviewer

Oncophysiology Group, Max-Planck-Institute of Experimental Medicine

Prof. Dr. Tobias Moser, Reviewer

Dept. of Otorhinolaryngology, Göttingen University Medical School Prof. Dr. Silvio Rizzoli

Dept. of Neuro- and Sensory Physiology, Göttingen University Medical School

Date of the oral examination: 22.04 .2016 
I hereby declare that this doctoral thesis has been written independently with no other sources and aids than those cited.

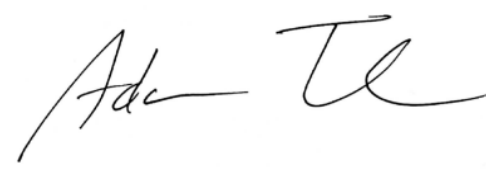

Adam Tomczak

01.03 .2016

Göttingen, Germany 
Make for thyself a definition or description of the thing which is presented to thee, so as to see distinctly what kind of a thing it is in its substance, in its nudity, in its complete entirety, and tell thyself its proper name, and the names of the things of which it has been compounded, and into which it will be resolved.

Marcus Aurelius 


\section{Acknowledgements}

First, I would like to thank Prof. Luis Pardo and Prof. Walter Stühmer for their supervision. I would also like to thank Jorge Fernandez, who was involved in some of the electrophysiological recordings and in proofreading. Furthermore, I am very grateful to Kerstin Dümke and Annett Sporning for invaluable technical help, as well as to Ute Rust for organizational assistance and to Ralph Schliephacke, who made sure that the electrophysiological apparatus was working properly.

Apart from that, I would like to thank Prof. Ehud Isacoff for the opportunity to do the VCF and single-molecule colocalization experiments at his department at UC Berkeley, as well as for many useful ideas. I would like to thank Shashank Bharill, who participated in the single-molecule imaging experiments. During my stay at Berkeley, Zhu Fu helped me with molecular biology and Sandra Weise with organizational issues. I learned VCF from Sasha Grimm and Daniel Clemens. M. Kienzler provided glycine maleimide for the blocking step in VCF experiments. I had very stimulating discussions with Reza Vafabakhsh, Shai Berlin, Raz Palty and Nathan Dascal.

I would also like to acknowledge all the organizational support I got from Michael Hörner and Sandra Drübe (IMPRS Neurosciences). I would like to thank Prof. Tobias Moser and Prof. Silvio Rizzoli for participating in my Thesis Committee, as well as Prof. Bert de Groot and Dr. Andreas Neef for participating in the final examination.

I am grateful to Carolina Leon and David Brockelt, who took the time to proofread the thesis and supported me in general, and to Ricardo Merino for interesting discussions and his positive attitude.

Finally, I would like to thank Diana Urrego-Blanco for helping me to put together this thesis and, more importantly, for her emotional support and teaching me a lot of new things. Te quiero mucho. 


\section{Table of Contents}

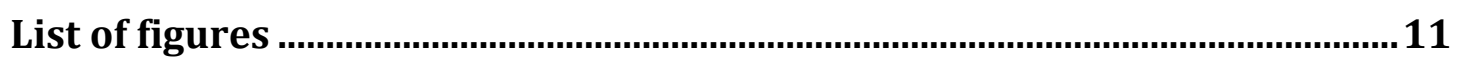

List of abbreviations .................................................................................13



Families of $\mathrm{K}^{+}$channels ............................................................................................

Structure of voltage-gated $\mathrm{K}^{+}$channels .........................................................................

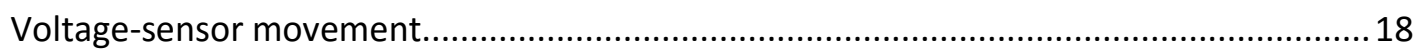

Proposed mechanisms of coupling between VSD and PD ............................................. 19

Thermodynamic perspective on voltage-gating ............................................................ 21

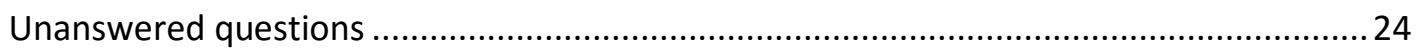

Split Kv10.1 channels as a tool to study voltage-gating.................................................... 24



Constitutive activity of channels interrupted close to 54 .............................................. 26

Dramatically altered kinetics of channels interrupted close to 55 ....................................... 28

Point mutations of S4-S5 linker rescue closure and deactivation kinetics of split channels

$\ldots$

Single molecule colocalization between fluorescently tagged VSD and PD ........................36

Breaks in S4-S5 linker affect VSD motion and coupling with PD ....................................... 38

Isolated pore module prefers the closed conformation.................................................... 42

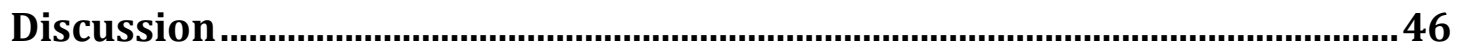

Constitutively active split channels............................................................................ 46

Changes of activation and deactivation kinetics .......................................................... 48

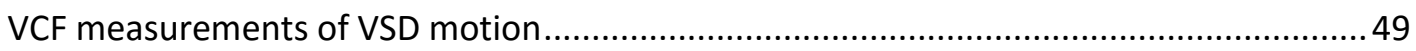

Intrinsic preference of the pore and a dual role of the VSD ...........................................50

Split channels from other families ............................................................................ 50

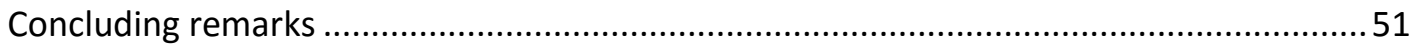

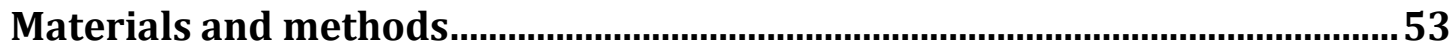

Mutagenesis and expression in Xenopus oocytes .....................................................53

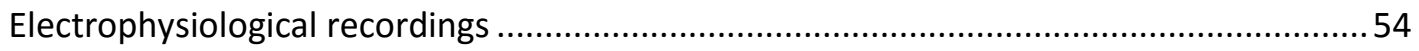

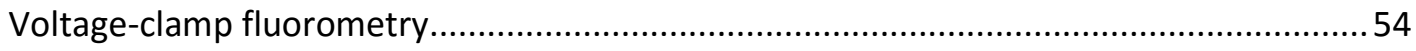



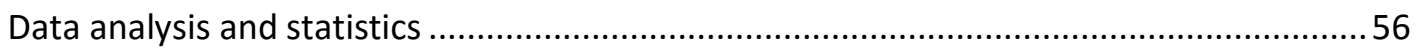



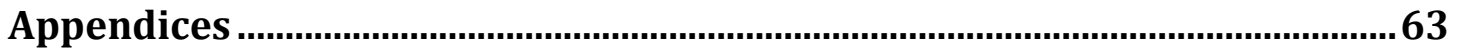




\section{List of figures}

Figure 1. Basic structural template of voltage-gated $\mathrm{K}^{+}$channels..................................17

Figure 2. Constitutive activity of specific split channels ..................................................... 26

Figure 3. Currents through constitutively active split Kv10.1 channels depend on

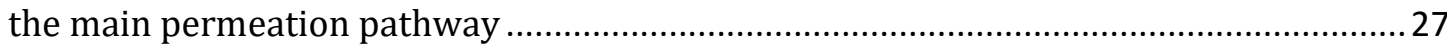

Figure 4. GV curves of the split channels that fully close...............................................28

Figure 5. Alterations of deactivation kinetics in Y347 split and G348 split......................29

Figure 6. Slower activation of Y347 split and G348 split ............................................... 30

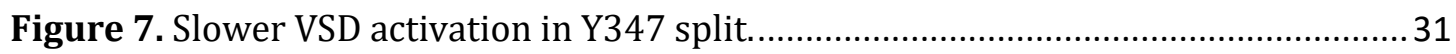

Figure 8. Restoration of wild-type-like closure with point mutations in S4-S5 linker.... 33

Figure 9. D342 mutants in the full-length channel............................................................ 34

Figure 10. Y347 mutations restore deactivation kinetics of Y347 split...........................35

Figure 11. Single molecule colocalization between fluorescently tagged VSD and PD. .. 36

Figure 12. Split channels remain functional after tagging with fluorescent proteins ......37

Figure 13. I319C mutation has only limited functional consequences..............................39

Figure 14. Voltage-dependency of conductance and VSD activation of specific split

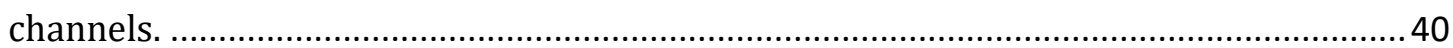

Figure 15. Effect of $\mathrm{Mg} 2+$ on current activation kinetics............................................... 41

Figure 16. Proline substitutions in inner S6 lock the full-length channel in the open state

Figure 17. Effects of Q476P mutation on PD expressed in isolation and with the VSD .. 43

Figure 18. Effects of Q477P mutation on PD expressed in isolation and with the VSD .. 44

Figure 19. Charge neutralizations in S4 can shift voltage-dependency of the channel to both positive and negative potentials 


\section{List of abbreviations}

\begin{tabular}{|c|c|}
\hline CaMBD & calmodulin-binding domain \\
\hline CNBHD & cyclic nucleotide-binding homology domain \\
\hline Eag1 & Ether-a-go-go Potassium Channel 1 \\
\hline EGFP & Enhanced Green Fluorescent Protein \\
\hline FRET & fluorescence resonance energy transfer \\
\hline FV & fluorescence-voltage \\
\hline GV & conductance-voltage \\
\hline hERG & human Ether-a-go-go Related Gene \\
\hline IV & current-voltage \\
\hline $\mathrm{KCNH}$ & Potassium Channel, Voltage Gated Eag Related Subfamily H \\
\hline LRET & luminescence resonance energy transfer \\
\hline MTS & methanethiosulfonate \\
\hline NLS & nuclear localization signal \\
\hline PAS & Per-Arnt-Sim \\
\hline PD & pore domain \\
\hline SD & standard deviation \\
\hline SEM & standard error of the mean \\
\hline TCC & tetramerizing coiled coil \\
\hline TIRF & total internal reflection fluorescence \\
\hline TMRM & tetramethylo-6-rhodamine maleimide \\
\hline VCF & voltage-clamp fluorometry \\
\hline VSD & voltage-sensing domain \\
\hline WT & wild-type \\
\hline
\end{tabular}




\section{Introduction}

Voltage-gated channels are essential for excitable tissues ${ }^{[1]}$. Their ability to switch from open to closed conformation upon small changes of transmembrane voltage underlies every nerve impulse and every muscle contraction. Thus, it is not surprising that the mechanisms of voltage-gating have received enormous attention of biophysicists since the existence of voltage-gated channels was predicted by Hodgkin and Huxley[2]. However, despite exhaustive research, some details of voltage-gating remain elusive. In this section, I will briefly review the current understanding of how voltage-gated $\mathrm{K}^{+}$channels work, define some of the unanswered questions in the field, and ask how split Kv10.1 (KCNH1, eag1) channels may contribute to answering them.

\section{Families of $\mathrm{K}^{+}$channels}

In total, around 70 genes in the human genome code for $\mathrm{K}^{+}$channels, 40 of them being voltage-gated channels. The other 30 code for inward-rectifying $\mathrm{K}^{+}$ channels, two pore $\mathrm{K}^{+}$channels and $\mathrm{Ca}^{2+}$-activated $\mathrm{K}^{+}$channels ${ }^{[3]}$. Such a variety of $\mathrm{K}^{+}$channels serves a number of physiological functions not limited to excitable tissues, where they repolarize the cell membrane after an action potential. For instance, $\mathrm{K}^{+}$channels have been strongly implicated in cell cycle and proliferation $^{[4]}$ (Appendix 2). After G-protein coupled receptors and protein kinases, voltage-gated $\mathrm{K}^{+}$channels are the third largest group of drug targets[5]. Interestingly, the channel pore is so well conserved that it can be successfully substituted from a prokaryotic to a eukaryotic channel[6]. Taken together, these observations suggest that the basic solution for $\mathrm{K}^{+}$permeation appeared relatively early in the evolution and was later adapted to many different physiological contexts.

\section{Structure of voltage-gated $\mathrm{K}^{+}$channels}

The extensive family of voltage-gated $\mathrm{K}^{+}$channels shares a basic structure of 4 subunits per channel and six transmembrane helices in each subunit. The first four helices form the voltage-sensing domain (S1-S4; VSD), and the remaining two 
belong to the pore domain (S5, S6; PD). A crucial part of the VSD is the S4 helix with an arrangement of positively charged amino acids that sense changes of transmembrane potential[7, 8]. Movement of these charges upon a change of voltage can be recorded as a transient gating current ${ }^{[9]}$.

Our understanding of $\mathrm{K}^{+}$channel structure has increased dramatically since the crystal structures of specific channels were solved by Roderick MacKinnon's group ${ }^{[10-12]}$. The central pore with a highly conserved selectivity filter formed by reentrant loops between PD helices is surrounded four VSDs, which protrude into the lipid bilayer. Electron densities for lipid molecules were found between VSD and PD, neighboring VSDs, and even between VSD helices, in line with the finding that voltage-gated ion channels can be regulated by membrane lipids ${ }^{[13,14]}$. Each VSD has two interfaces with PD: the S4-S5 linker and a surface between the top (extracellular) part of S1 and S5 of the adjacent subunit. Interestingly, the subunits are interwoven in such a way that the S4-S5 linker passes under the PD helices of the neighboring subunit before it connects with the S5 helix of its own subunit. Thus, the VSD contacts pore helices of its own subunit (through S4-S5 linker), as well as of the neighboring subunit (through S1-S5 interface), allowing cooperative inter-subunit interactions.

Within the VSD, one can distinguish a helix-turn-helix motif consisting of Cterminal S3 and S4, named as the voltage-sensing paddle. Remarkably, a large part of the paddle is exposed to the lipid bilayer. As proposed by MacKinnon and colleagues, the paddle undergoes a large movement relative to the rest of the VSD when transmembrane voltage changes. Surprisingly, the paddle can tolerate large deletions, the most radical of which replaces 43 amino acids (including the first two voltage-sensing arginines) with a glycine triplet[15]. Furthermore, in Kv1.2-2.1 chimera and several other Kv channels, the S6 helix contains a Pro-Val-Pro kink; the bending of inner S6 below the kink regulates access to the pore. However, as of today, no crystal structure of a voltage-gated $\mathrm{K}^{+}$channel in the closed state has been solved. Consequently, inference of mechanisms from the available structural data is based on one conformation only. It is also possible that some of the available crystal structures might represent a non-permeating conformation 
distinct from the closed state (the slow-inactivated state), which may have different S4 position than the open state.

a

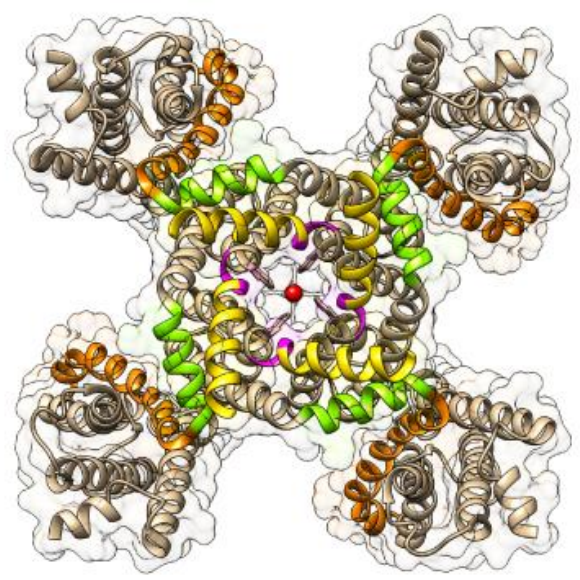

b

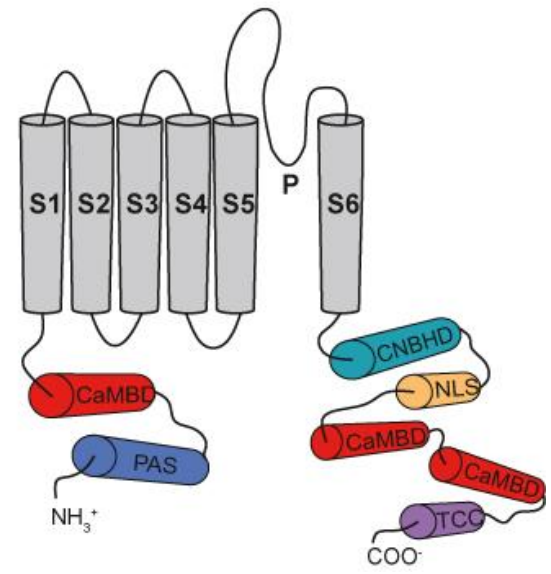

Figure 1. Basic structural template of voltage-gated $\mathrm{K}^{+}$channels. (a) Crystal structure of Kv1.2-2-1 chimera (PDB code 29R9) viewed from the intracellular side. The parts of the gating machinery are color-coded: S4 (orange), S4-S5 linker (green), inner S6 (yellow), PVP kink (magenta). (b) A schematic representation of Kv10.1 subunit with intracellular domains: Per-Arnt-Sim (PAS) domain, calmodulin-binding domains (CaMBD), cyclic nucleotide-binding homology domain (CNBHD), nuclear localization signal (NLS) and tetramerizing coiled coil (TCC).

Although Kv 1.2-2.1 chimera is probably a good general reference for Kv10.1, the crystal structure of which has not been solved so far, there are some important differences that should be mentioned. First, Kv10.1 has distinct intracellular domains, compared to other voltage-gated channels outside $\mathrm{KCNH}$ family: Per-Arnt-Sim (PAS) domain, calmodulin-binding domain and cyclic nucleotide-binding homology domain (CNBHD), which influence channel gating and have been related to certain disease outcomes. Notably, the only structural data available for Kv10.1 are the crystal structures of the PAS domain and CNBHD in isolation $[16,17]$ and together in a complex, revealing an interaction interface between them, which is a hotspot for disease-related mutations[18]. However, the orientation of the cytoplasmic domains with respect to the transmembrane helices is unknown. Second, S6 helices of KCNH channels do not have a Pro-Val-Pro kink. Moreover, they do not include any helix-breakers at all. Hence KCNH channels might have a different gating mechanism than rotation of inner S6 around a pivot, which has been proposed for Shaker and the related channels. It should also be 
mentioned here that the homology level is far too low to use the fine structural details of Kv1.2-Kv2.1 chimera to design or interpret experiments on KCNH family channels.

\section{Voltage-sensor movement}

In order for the VSD to serve its function, voltage-sensing charges must move upon a change of transmembrane electric field. As mentioned above, their movement can be detected as gating current. Integration of gating current with respect to time, together with an estimate of the number of channels in the preparation allow to calculate the total gating charge per VSD, equal to 3-3.5 elementary charges in Shaker[19-21]. This estimate fits the number of basic residues in S4 very well. However, uncovering of the underlying VSD motion required further studies. Because the transmembrane electric field can be focused by invaginations of the channel protein, the extent of S4 motion does not have to correspond to the membrane thickness. Even a small S4 movement could result in a full charge transfer if the field is very focused. On the other hand, voltage-sensing might require a large S4 motion. These alternatives have drawn a lot of attention and inspired a host of creative experimental approaches, which did not converge on a single conclusion.

One strategy involved measuring accessibility of S4 residues from both sides of the membrane to impose upper and/or lower limits on S4 motion. An assay based on binding of biotin tethered with linkers of various lengths to avidin, which is too large to fit inside invaginations in the channel protein, suggested a large motion of $15-20 \AA$ [22, 23]. An upper limit of about $13 \AA$ was found in experiments that employed conjugation of small, charged methanethiosulfonate (MTS) reagents ${ }^{[24]}$. A much smaller S4 motion was reported by fluorescence and luminescence resonance energy transfer measurements (FRET, LRET)[25, 26]. In those experiments, the donor fluorophore was attached at various positions in the channel, including S4, and the acceptor served as a reference, located either in the membrane or conjugated to a toxin sitting in the central pore. Both studies estimated the vertical component of S4 motion at around $2 \AA$ and postulated that the transmembrane electric field is highly focused. 
The idea that VSD can focus electric field is supported by the findings that, under some conditions, the VSD might conduct protons ${ }^{[27]}$ or larger ions ${ }^{[28]}$. For permeation through VSD to occur, a gating pore must exist, whereby two waterfilled cervices are separated by a distance considerably shorter than the membrane thickness. Another line of evidence that points to field focusing comes from restoration of gating charge, which is lost after mutating the first voltagesensing arginine to a cysteine, with positively charged MTS reagents[29]. In this study, tethers of various lengths were used with the idea that at some point the tether will be too long for the MTS reagent to recover the lost gating charge. Indeed, charge restoration turned out to be a sigmoidal function of tether length and it was estimated that $4 \AA$ motion is sufficient for the first voltage-sensing arginine to cross the entire transmembrane field.

While it is possible that some of the approaches underestimate or overestimate VSD motion, it is not straightforward to find a consensus between them. With some assumptions, an intermediate estimate of around $8 \AA$ can be used to reconcile most of the available experimental data[30]. Also the computational studies converge on a motion of $10 \AA \AA^{[31]}$. This would mean that the electric field is very focused, but VSD movement is greater than reported by FRET experiments.

\section{Proposed mechanisms of coupling between VSD and PD}

VSD motion must be translated to open and closed conformations of the channel pore. It has been generally accepted that S4-S5 linker is a crucial interface for electromechanical coupling between VSD and PD. However, the exact mechanism of coupling is still under debate.

Extensive evidence for the importance of S4-S5 linker came from mutagenesis studies, in which perturbations of the linker led to alterations of voltage-dependency and kinetics of several channels [32-37]. In addition, Zhe Lou and colleagues found that the bacterial channel KcsA, which is gated by protons and only weakly voltage-sensitive, can be endowed with voltage-dependency by transplantation of the VSD and the C-terminal S6 helix from Shaker[38]. Remarkably, the complementarity between S4-S5 and inner S6 sequences was a requisite for the resulting chimeras to be functional and/or voltage-dependent. 
This finding was further reinforced by similar results from experiments that replaced the S4-S5 linker and the C-terminal portion of S6 helix in Kv1.5 with their counterparts from Kv2.1[39]. An important interaction between the N-terminal part of the S4-S5 linker and the channel gate in S6 was also described in Kv11.1 (hERG) ${ }^{[40,41]}$. At negative potentials, the residues D540 and R655 form a salt bridge, which stabilizes the closed conformation of the channel.

The crystal structures, in combination with the functional evidence described above, inspired a very intuitive model of voltage-gating[12,42]. Given that in the open conformation S4-S5 linkers of the four subunits form a ring around inner S6 helices, it is tempting to assume that, upon membrane hyperpolarization, inward movement of S4-S5 linkers exerts a force on inner S6 helices, constricting and closing the pore. The motion predicted by this model would somehow resemble a closing diaphragm of a camera. Inner S6 helices move together with S4-S5 linkers, while outer S6 remain stationary, owing to the Pro-Val-Pro kink, which breaks S6 in two parts and serves as a pivot. In turn, when the membrane is depolarized, outward movement of the S4-S5 linker would ease the tension on the inner S6 bundle, allowing the pore adopt the open conformation. Another plausible scenario is that inner S6 follows the outward movement of S4-S5 linker, because of non-covalent interactions between them.

By contrast, a recent state of the art all-atom molecular dynamics simulation of $\mathrm{Kv} 1.2 / 2.1$ paddle chimera revealed a different mechanism of voltage-dependent gating[43]. In the simulation, it was not necessary for the S4-S5 linker to maintain the pore shut at negative potentials, because hydrophobic collapse of the pore occurred spontaneously (further evidence for hydrophobic collapse comes from simulations of PD in isolation[44]) On the contrary, depolarization-driven work was needed for channel opening at positive potentials. Upon depolarization, the channel switched from resting, VSD-pore loosened conformation to active, VSD-pore apposed conformation. At depolarized potentials, both electrostatic and Leonard-Jones interactions between linker and gate residues became more energetically favorable and the number of contacts between the linker and the gate increased. Repacking of S4-S5 and inner S6 was thus required to open the pore. 
In conclusion, we are still far from a coherent, mechanistic explanation of electromechanical co in voltage-gated $\mathrm{K}^{+}$channels. The key components of the coupling machinery have been identified, but the details of this fascinating process remain obscure. Without any knowledge of $\mathrm{K}^{+}$channel structure, Hodgkin and Huxley concluded that activation of voltage-dependent $\mathrm{K}^{+}$conductance requires movement of four voltage-sensing particles ${ }^{[2]}$. This can be translated to an obligatory gating model, in which all four VSDs need to be activated before the pore can open in a final cooperative step. This has been demonstrated for Shaker and closely related channels [45-47]. However, recent experiments in KCNQ channels point to an allosteric gating mechanisms, with the movement of each sensor individually contributing to the probability of finding the channel in the open state ${ }^{[48,49]}$. It is thus also possible that gating mechanisms vary between channel families.

\section{Thermodynamic perspective on voltage-gating}

In most general terms, VSD movement changes the energy landscape of the

channel protein, allowing it to switch between open and closed conformations of the pore. Boltzmann distribution law states that in a system with many states the probability of a given state is proportional to the energy associated with it:

$$
P_{i} \propto e^{-\frac{E_{i}}{k T}},
$$

where $P_{i}$ is the probability of a given state to occur, $E_{i}$ the energy associated with it, $k$ is the Boltzmann constant, and $\mathrm{T}$ is the absolute temperature. When fitting a sigmoidal GV curve, one assumes only two states of the channel protein: closed and open. The probability of the open state is equal to:

$$
P o=\frac{e^{-\frac{E o}{k T}}}{e^{-\frac{E o}{k T}}+e^{-\frac{E c}{k T}}}=\frac{1}{1+e^{-\frac{E c}{k T}}},
$$

where $E_{o}$ and $E_{c}$ are the energies associated with the open and the closed conformation, respectively. It follows that when the energy of the closed state is 
low, the open probability must also be low. Conversely, when the energy of the closed state is high, open probability is close to 1 . Given that:

$$
k=\frac{R}{N a} \quad E=z F V \quad F=e N a,
$$

where $k$ is the Boltzmann constant, $R$ is the gas constant, $N a$ is the Avogadro number, $E$ is the electrical potential energy, $z$ is the apparent charge valence, $V$ is the voltage, $F$ is the Faraday constant and $e$ is the elementary charge, the equation for open probability can be rewritten as follows:

$$
P o=\frac{1}{1+e^{-\frac{z F}{R T}\left(V-V_{1 / 2}\right)}}
$$

This is the function used to fit conductance-voltage curves in most studies in the field, as well as in this dissertation. $\mathrm{V}_{1 / 2}$ is the voltage for which the open probability equals 0.5 . However, the assumption that the channel only exists in two states in an oversimplification, because there can be more closed and open states. For instance, in Kv10.1, VSD movement and channel opening become slower after a hyperpolarizing prepulse or upon $\mathrm{Mg}^{2+}$ binding to the VSD [50,51], which implies that the VSD can adopt several conformations in the closed state. Thus, two-state Boltzmann function should not be used to estimate the energy difference between the closed and the open state. Multistate models have also been used, but the choice how many states to include in such a model is always somehow arbitrary and will obviously affect the parameters. Sometimes, the same set of experimental data can be predicted by different combinations of model parameters and their true value cannot be estimated[52]. For this reason, it would be very useful to have model-independent parameters, such as the median voltage of charge movement proposed by Baron Chanda's group[53]. Interestingly, the energy associated with channel opening in Shaker was estimated at -2 to -3 $\mathrm{kcal} / \mathrm{mol}$ with a two-state Boltzmann function and at $-14 \mathrm{kcal} / \mathrm{mol}$, using the median voltage of charge transfer. The latter is much more realistic, because, if the former were true, a change of a single non-covalent interaction in any of the four subunits could make the channel switch between open and closed conformations. Optimally, one would measure structural changes associated with gating, with a 
method that does not rely on ionic or gating currents, like single-molecule FRET[54]

Another interesting question, which we already briefly touched upon, is whether VSD and PD are positively or negatively coupled[55]. VSD movement might be necessary to change the energy landscape, so that the pore can open at positive potentials, or to prevent it from opening at negative potentials. In other words, the interaction between VSD and PD could be attractive or repulsive, depending on which conformation is preferred by the pore in isolation. Increased separation between curves describing voltage-dependencies of VSD activation and conductance has often been interpreted as evidence for decoupling between VSD and PD. This holds true if coupling between them is positive. However, if coupling between them were negative, a larger voltage gap between VSD activation and conductance would actually suggest an increase in coupling strength. An argument often used to make case for negative coupling is that the channel adopts the open conformation in the absence of electric field. Indeed, Shaker and Kv1.2-2.1 chimera are open at $0 \mathrm{mV}$, whereas some other channels have their voltagedependency shifted to more positive values. Kv10.1, for example, reaches between 30 and $40 \%$ of its maximal open probability at $0 \mathrm{mV}$ (its maximal absolute open probability is less than 1 , so $0.3-0.4$ at $0 \mathrm{mV}$ is actually an upper estimate). Besides, it is hard to infer from the open conformation at $0 \mathrm{mV}$ alone that the channel pore would also be open at negative potentials if it were not under constant strain from the VSD. To reach this conclusion, one would need to show that the pore prefers the opens state in the absence of VSD. Indeed, sensorless PD of bacterial channel KvLM can open (albeit with a low open probability) and retains some rudimentary voltage-dependency, when purified and reconstituted into lipid bilayers ${ }^{[56]}$. However, the crystal structure of sensorless KvLM revealed the pore in a closed state [57]. Interestingly, purification and electrophysiological characterization of KvLM tetramers containing 0-4 VSDs showed that VSD is required for complete closure, as well as to stabilize the open state[58]. Pores of several bacterial $\mathrm{Na}^{+}$ channels remain functional after truncation of the $\operatorname{VSD}[59,60]$, and a sensorless pore module belonging to one of them has been crystalized in the open conformation[61]. On the overall, these result suggest that the pore intrinsically 
prefers the open conformation in specific bacterial channels. Beyond doubt, more experiments are needed to probe the interaction between VSD and PD in eukaryotic voltage-gated channels.

\section{Unanswered questions}

Our understanding of voltage-gated ion channels could clearly be improved. The main weakness of mutagenesis studies, which have been the working horse in the field, is that they oftentimes offer limited mechanistic explanations. Owing to insights from the available crystal structures, several important developments have been made, such as identification of the gating charge transfer mechanisms in the VSD[62,63]. However, until a reliable crystal structure of the closed state is solved, we are still in the dark about important aspects of how VSD motion is transferred to the channel gate. To make things more complicated, some of the unanswered questions are interconnected. For instance, any model of electromechanical coupling will necessarily depend on how far S4 is believed to move. Models that assume a limited S4 motion should not be dismissed, since the strength of non-covalent interactions falls rapidly with distance. Thus, even a modest protein motion could disrupt or form non-covalent interactions that bias the channel towards open or closed conformation. Along these lines, LRET experiments have estimated that a $4 \AA$ radial displacement of the S4-S5 linker is sufficient to close the channel pore of KvAP[64]. Irrespectively of the extent of motion of the gating machinery, it would be highly desirable to know the strength and the state-dependency of interactions at the interface between VSD and PD, as well as their structural constraints. Finally, more attention should be given to the question which conformation the pore prefers in isolation. We need more carefully designed experiments and more tools to address these points. We should also be open to the possibility that some features of voltage-gating, like the intrinsic preference of the pore, might be channel-specific.

\section{Split Kv10.1 channels as a tool to study voltage-gating}

Alongside with Kv11.1, Kv10.1 is the most widely known member of KCNH subfamily of voltage-gated $\mathrm{K}+$ channels. In terms of its physiological role, Kv10.1 
influences cell cycle progression[65], which is most likely why it is expressed by various tumors ${ }^{[66]}$. Apart from that, mutations that affect Kv10.1 gating have been linked with certain other disease outcomes $\left[{ }^{[67,68] . ~ K v 10.1 ~ i s ~ a l s o ~ e x p r e s s e d ~ i n ~ t h e ~}\right.$ brain, where it affects the activity of some synapses ${ }^{[69]}$. Our laboratory has previously shown that VSD and PD expressed as separate proteins co-assemble and form functional-voltage gated Kv10.1 channels in Xenopus oocytes, which recapitulate the voltage-sensing and permeation characteristics of the full-length channel[70]. This unexpected finding raises a host of further questions. What are the forces driving the assembly of these complexes? Do they have the expected stoichiometry of 8 parts? How is information transferred between domains of the protein that are not covalently bound? Are precise functional interactions between VSD and PD preserved in split channels? With such questions in mind, we realized that split channels might be used as a magnifying glass to examine different gating models. First, some gating models seem difficult to reconcile with functional split channels. For instance, it is unlikely that S4-S5 linker pulls on inner S6 to open the pore, because such a motion could hardly be effective when the linker is not continuous. Second, split channels enable experiments which could potentially be very relevant for the unanswered questions in the field and help dissect the steps of the gating pathway. More specifically, we started the project with the following objectives:

(i) electrophysiological characterization of split channels interrupted at different positions in the S4-S5 linker.

(ii) comparison of S4-S5 linker mutations in the full-length and split channels.

(iii) investigation of VSD-PD assembly at the single-molecule level.

(iv) evaluating how disconnection between VSD and PD affects VSD motion.

(v) checking the effect of S6 mutations that lock the full-length channel in the open state on PD expressed alone and together with the VSD. 


\section{Results}

\section{Constitutive activity of channels interrupted close to S4}

In light of our previous finding of functional, voltage-gated Kv10.1 channels that lack a covalent bond between VSD and PD ${ }^{[70]}$ (Appendix 3), we asked if disrupting the channel in different positions between S4 and S5 gives various outcomes or it uniformly leads to split channels that resemble the wild-type. To answer this question, we generated a series of split channels, removing the covalent bond after every residue from K340 to A349 (Fig. 2a). Each of the split channels was heterologously expressed in Xenopus oocytes and characterized with twoelectrode voltage clamp.
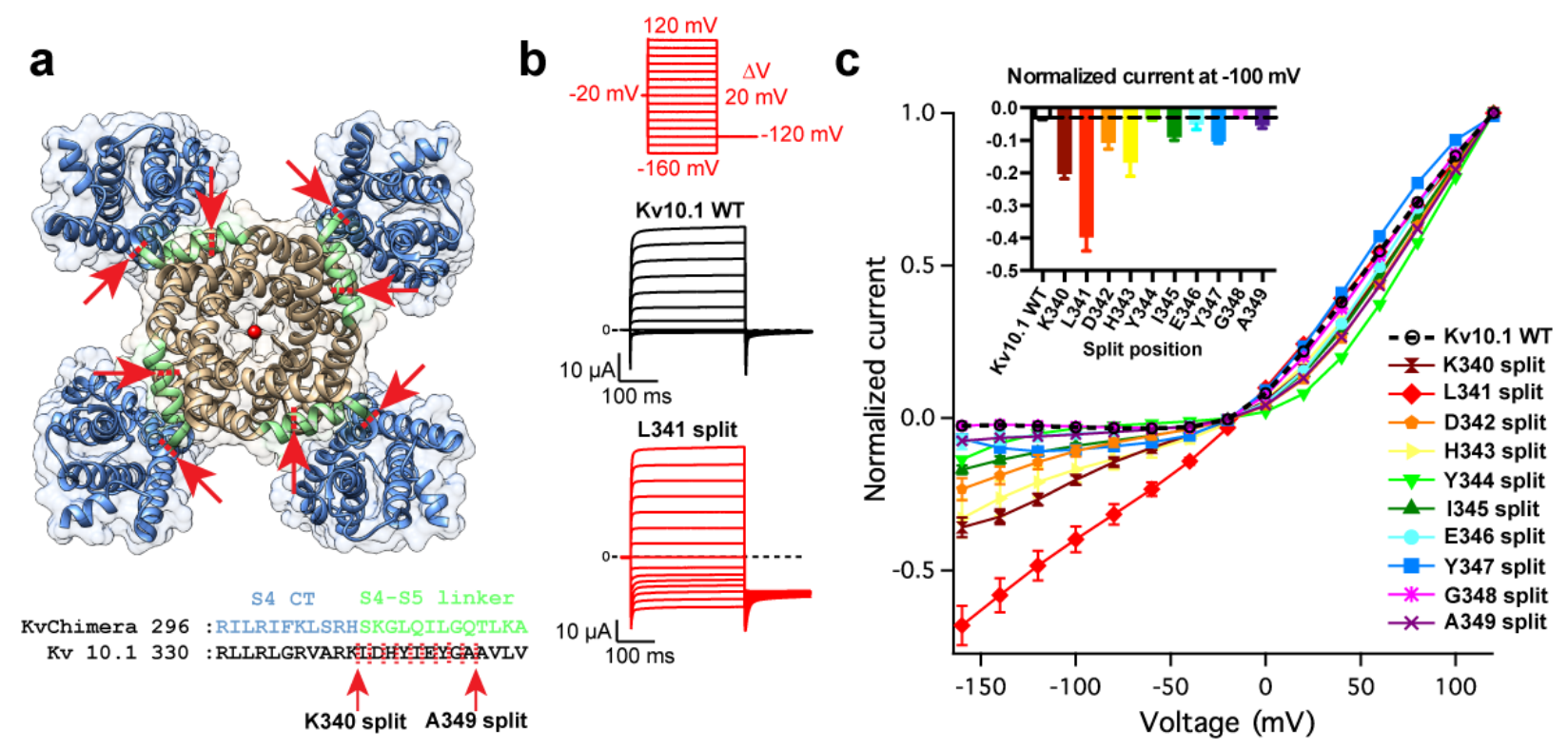

Figure 2. Constitutive activity of specific split channels. (a) The crystal structure of Kv1.2-2.1 chimera (PDB code 29R9) and comparison of C-terminal S4 and S4-S5 linker sequences between the chimera and Kv10.1. In this study, VSD was disconnected from PD after every residue between the positions shown by red arrows. (b) Representative, macroscopic currents of the wild-type and L341 split channels recorded with the indicated voltage protocol (without leak subtraction). (c) Normalized, average current-voltage curves of the wild-type and the split channels ( $n=6-14)$. Error bars denote SEM. Inset: Normalized current at $100 \mathrm{mV}$ plotted against the split position (mean \pm SEM), dashed line shows the current level in the wild-type. 
Strikingly, interrupting S4-S5 linker close to S4 (after residues K340 and L341) resulted in channels that were constitutively active, as indicated by large inward currents at negative potentials, which were absent when the break was closer to S5 (after residues E346, Y347, G348 and A349) (Fig. 2b, c). In between, we observed intermediate cases with some constitutive activity (after residues: D342, H343, Y344 and I345).
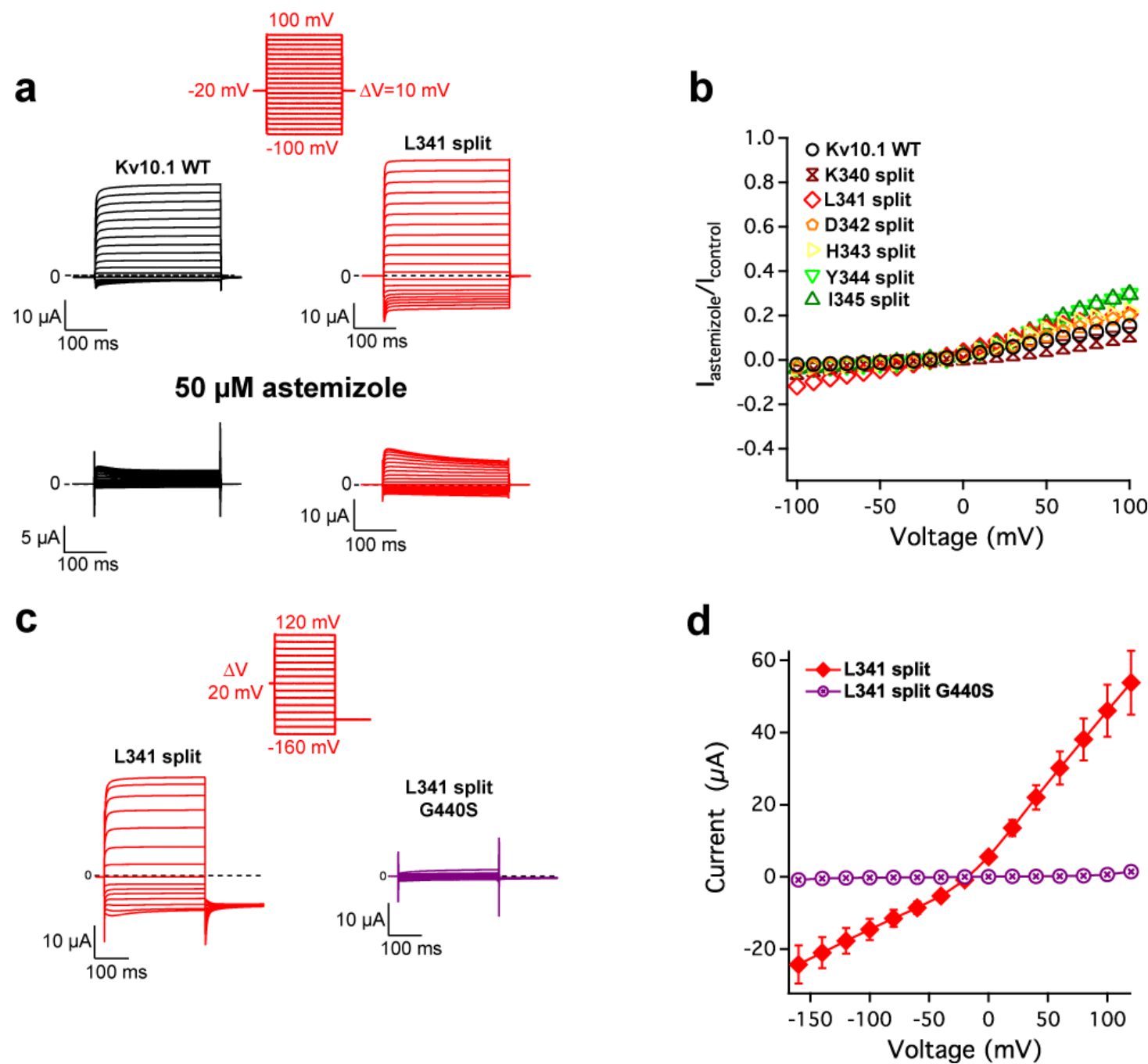

Figure 3. Currents through constitutively active split Kv10.1 channels depend on the main permeation pathway. (a) Representative currents of the wild-type and L341 split recorded with the indicated voltage protocol before and after application of $50 \mu \mathrm{M}$ astemizole. (b) The ratio of mean currents after and before application of $50 \mu \mathrm{M}$ astemizole plotted against voltage $(n=8-13)$. Error bars (SEM) are smaller than symbols. (c) Representative currents of L341 split with and without G440S mutation which renders the main pore non-conducting. (d) The corresponding average currents plotted against voltage $(n=12$; without normalization). Error bars denote SEM. 
Both outward and inward current through channels that had constitutive activity could be blocked by the Kv10.1 blocker astemizole and disappeared in experiments which used a non-conducting PD, indicating that no flux of ions through the gating pore in the VSD is involved (Fig. 3). Interestingly, we also found that the split channels that which close, with the exception of Y347 split, either had the conductance-voltage (GV) curve shifted to more positive values or showed a biphasic dependency of open probability on voltage with one not saturated component (Fig. 4). Considered together, these results imply that discontinuity between VSD and PD can energetically bias the channel towards both open and closed conformations.

a

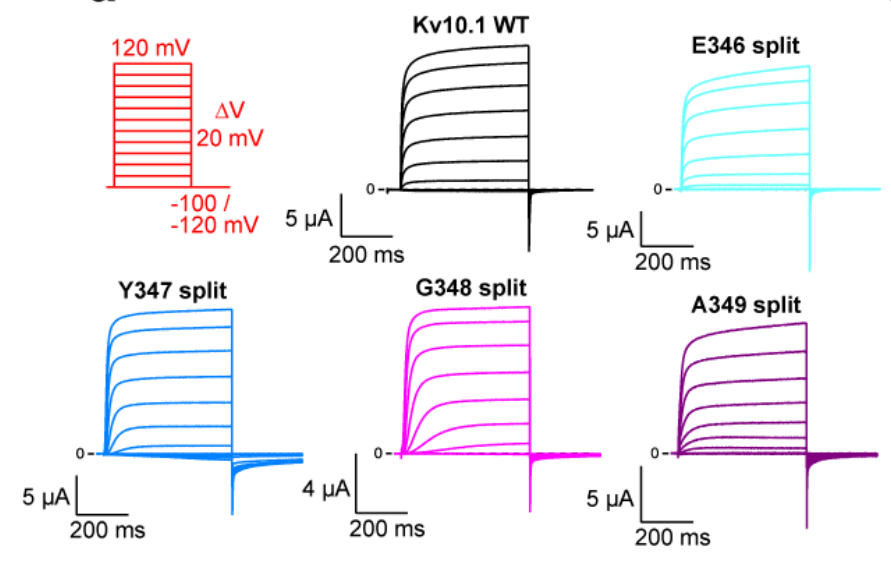

b

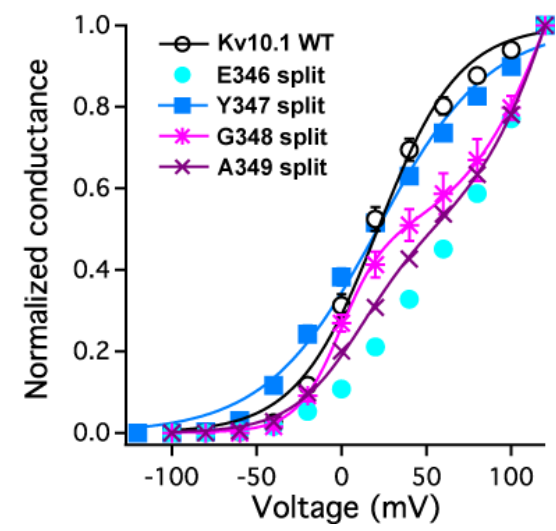

Figure 4. GV curves of the split channels that fully close. (a) Representative currents of the wild-type and the split channels which have no or very little constitutive activity recorded with the voltage protocol shown (with leak subtraction). (b) Normalized conductance plotted against voltage. Data points represent mean \pm SEM $(n=8-10)$, the curves are single or double Boltzmann fits.

\section{Dramatically altered kinetics of channels interrupted close to S5}

Incidentally, we observed radical alterations of kinetics in the channels split after Y347 and G348. Although Y347 split could close completely, deactivation took up to $20 \mathrm{~s}$, whereas it did not exceed $300 \mathrm{~ms}$ in the wild-type channel (Fig. 5a). Remarkably, the fast deactivation time constant was nearly overlapping between the wild-type, Y347 split and G348 split (Fig. 5b, c), but Y347 split had an additional super-slow component with some voltage-dependency, as complete 
closure was faster at more negative potentials. G348 split also showed slower deactivation, but the effect was smaller than in Y347 split.

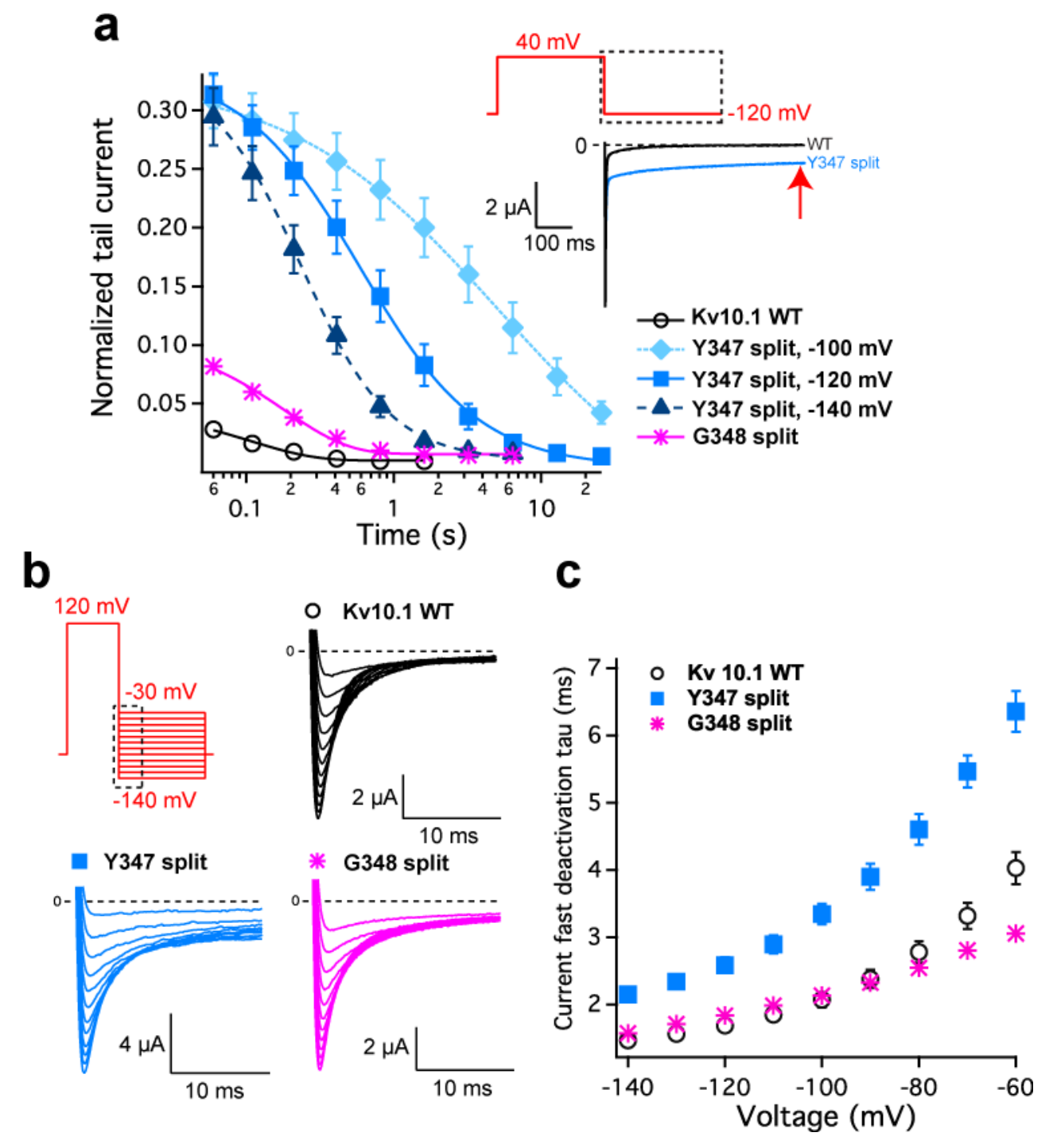

Figure 5. Alterations of deactivation kinetics in Y347 split and G348 split. (a) Tail current (normalized to the steady-state current at the end of the depolarizing segment of the voltage pulse) plotted against time at negative potential (mean \pm SEM; $n=6$ ). Upper right: super-slow deactivation component shown by the red arrow. (b) Representative tail currents recorded at different potentials with the protocol shown in the upper right corner. (c) Fast deactivation time constants obtained by double exponential fits to tail currents (mean \pm SEM; $n=7-8)$.

To study activation kinetics, we employed a reactivation protocol, in which channels were opened with a pulse to $+40 \mathrm{mV}$, then stepped to $-120 \mathrm{mV}$ for a variable time and opened again with another pulse to $+40 \mathrm{mV}$ (Fig. 6a). This 
protocol allows to trace entry into deeper closed states, reflected by delayed current activation as a function of interpulse interval at negative potential.

a
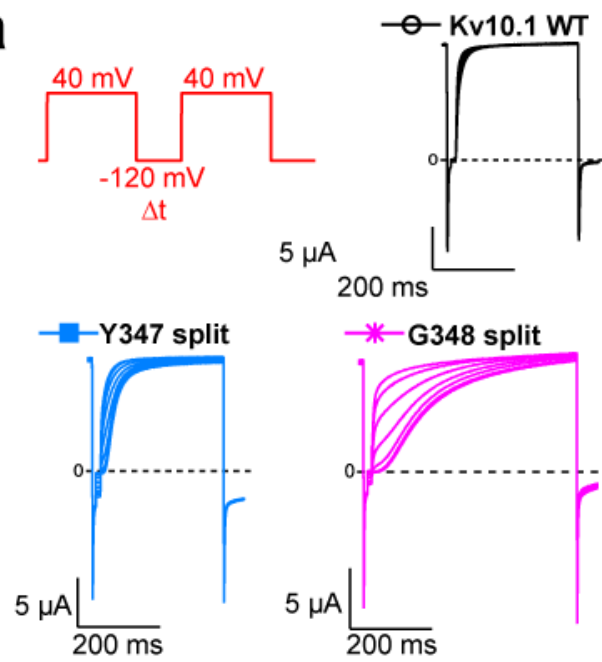

C
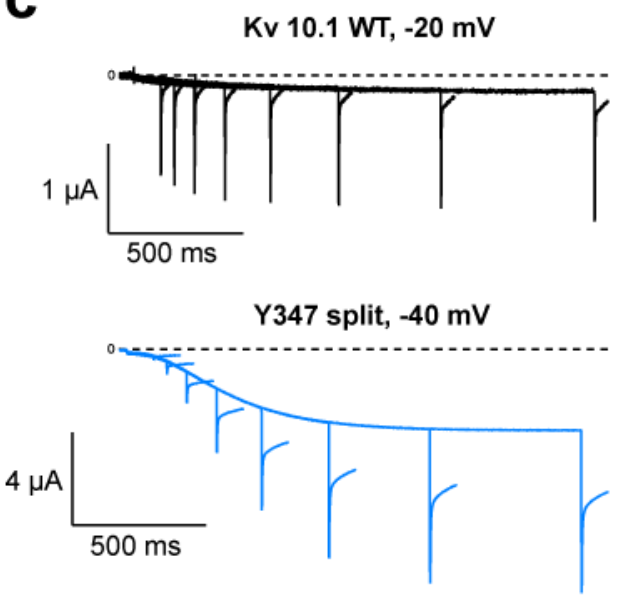

b

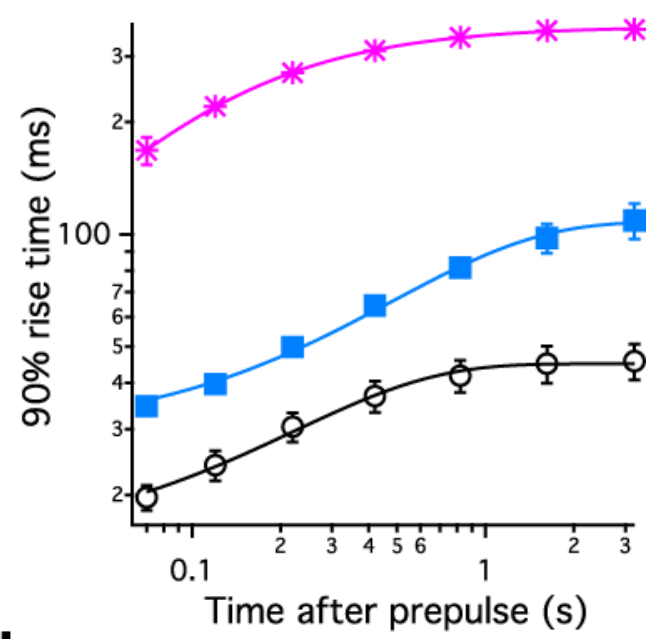

d

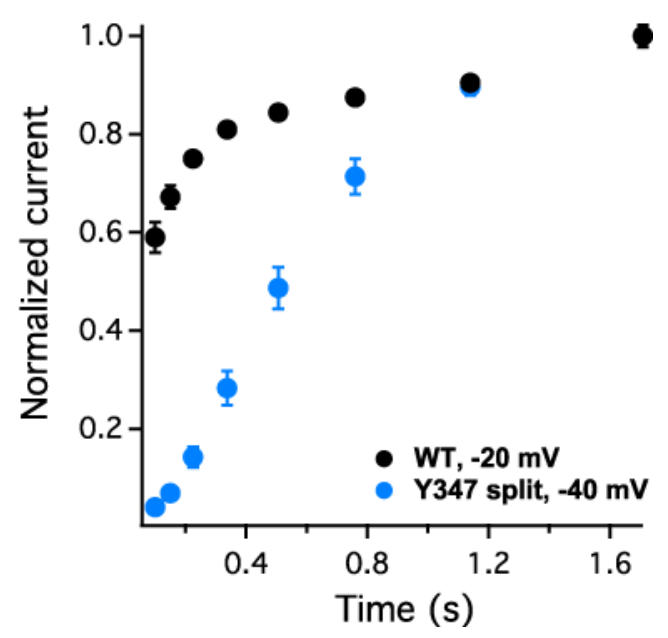

Figure 6. Slower activation of Y347 split and G348 split (a) Representative currents of the wild-type, Y347 split and G348 split recorded with the indicated reactivation protocol, with various intervals at $-120 \mathrm{mV}$ between the depolarizing pulses. (b) Time necessary for current to reach $90 \%$ of its maximal value plotted against the length of the interval at $-120 \mathrm{mV}$ (mean \pm SEM; $\mathrm{n}=6-8$ ). (c) Representative currents elicited by voltage pulses of increasing duration to $-20 \mathrm{mV}$ (Kv10.1 WT) and -40 mV (Y347 split). (d) Normalized tail current plotted against the length of the voltage pulse (mean \pm SEM; $n=3-6$ ).

Even though some channels remained open at the end of the negative segment of the pulse (because of super-slow deactivation), Y347 split activated slower at every time interval, compared to the WT (Fig. 6b). Slower activation of G348 split was yet more pronounced, with all rise times in being an order of magnitude 
higher than in the wild-type. Moreover, at voltages slightly above the threshold of the GV curve, Y347 split took more than a second to reach steady-state current, whereas the wild-type needed less than 500 ms (Fig. 6c, d).

We next asked if the altered kinetics of Y347 split, which showed major changes in both activation and deactivation, could be explained by a change in VSD movement. To this end, we introduced I319C mutation, which reports on voltagesensing motions in Kv10.1 when labeled with tetramethylo-6-rhodamine maleimide (TMRM) [51]. We also tried other labeling sites in the vicinity (G318S322) and found that I319C gives the best signal.
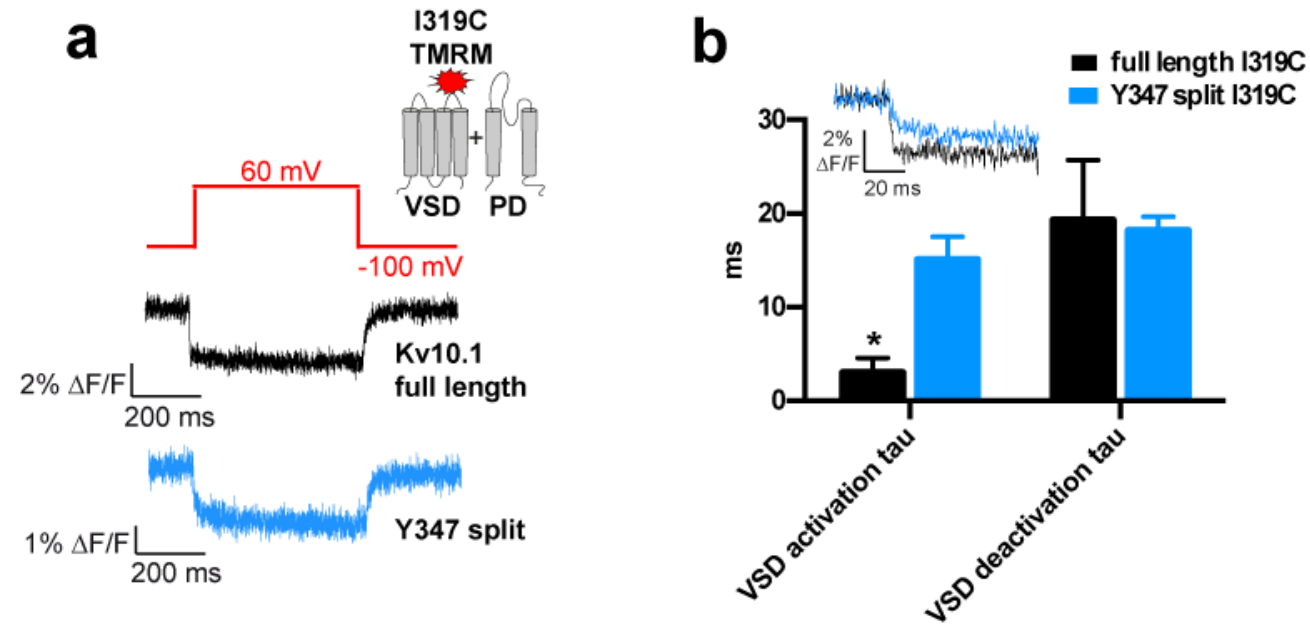

Figure 7. Slower VSD activation in Y347 split. (a) Representative voltagedependent changes of fluorescence reported by I319C-TMRM and elicited by a depolarization to $60 \mathrm{mV}$ from a holding potential of $-100 \mathrm{mV}$ (b) Activation and deactivation time constants obtained by exponential fits to fluorescence traces of the full-length channel and Y347 split ( $n=5$-8; mean \pm SD). Inset: superimposed VCF traces from (a) with an expanded time base.

We quantified the kinetics of the Voltage-Clamp Fluorometry (VCF) signal elicited by a voltage step from -100 to $60 \mathrm{mV}$ (Fig. 7a). The activation time constant was significantly faster in the full-length channel than in Y347 split $(3.0 \pm 1.5 \mathrm{~ms}$ and 15.1 $\pm 2.4 \mathrm{~ms}$; mean $\pm \mathrm{SD}, \mathrm{p}<1 \times 10^{-6}$, T-test), whereas the deactivation time constant was similar in both cases $(19.3 \pm 6.4 \mathrm{~ms}$ and $18.2 \pm 1.4 \mathrm{~ms}$, respectively) (Fig. 7b). It is worth mentioning that the activation time constant of Y347 split is probably underestimated, because the need to average traces forced very short interpulse intervals at negative holding potential, and the activation kinetics might 
depend on the length of that interval. Thus it would seem that slower current activation in Y347 split can be at least partly attributed to slower VSD movement, but the super-slow deactivation component remains unexplained.

\section{Point mutations of S4-S5 linker rescue closure and deactivation kinetics of split channels}

Disconnection between VSD and PD could change biophysical properties of the channel due to a gross structural change. Alternatively, precise functional interactions of S4-S5 linker amino acids with their counterparts in the pore domain or in the cytoplasmic domains could be disturbed. If a change spanning large regions of the channel protein is involved, it is unlikely that wild-type-like properties could be restored by point mutations of linker amino acids. To test that prediction, we first turned to mutagenesis of Asp 342, because the homologous Asp 540 plays a role in channel closure in Kv11.1[40, 41].

We set out to check if constitutive activity of L341 split could be reduced by mutating D342 to 12 amino acids with different physicochemical properties. Strikingly, all of them, except for Asn, which is isosteric with Asp, greatly facilitated closure, irrespectively of side chain size, charge and hydrophobicity (Fig. 8). Notably, at $-100 \mathrm{mV}$ only three split channels with Asp, Asn and Leu at 342 positon had significantly more current than the wild-type (Bonferroni's multiple comparisons test; interestingly, at $-160 \mathrm{mV}$ nearly all split channels tested had more current than the WT, suggesting that they might reopen at very hyperpolarized voltages). Also deletion of D342 or longer deletions that started from D342 restored closure. In line with these observations, the corresponding D342 mutations in full-length channel, with the exception of Trp, caused a rightward shift of the GV curve (Fig. 9). D342N mutant showed only a slight positive GV shift, with respect to the wild-type $(10 \mathrm{mV}$, the intervals encompassing the mean \pm 3 SD overlap with the WT). D342W mutation, in turn, resulted in channels that were locked-open, suggesting steric hindrance between the bulky Trp residue and the channel gate. However, none of these occurred when Trp was substituted at this position in the split channel, indicating that the position of S4S5 linker might be different than in the full-length protein. 


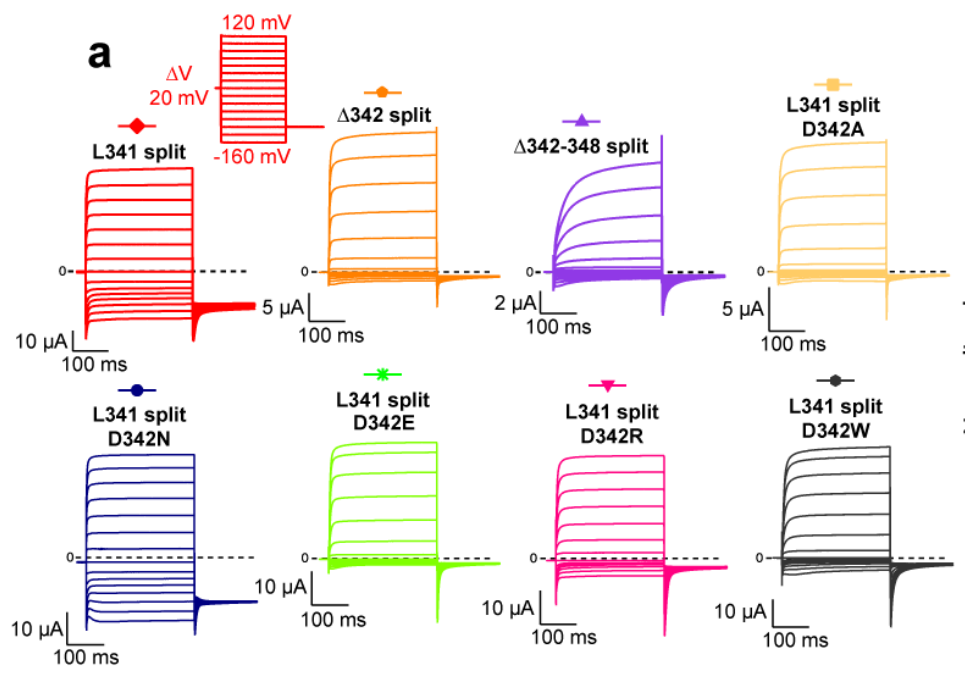

b

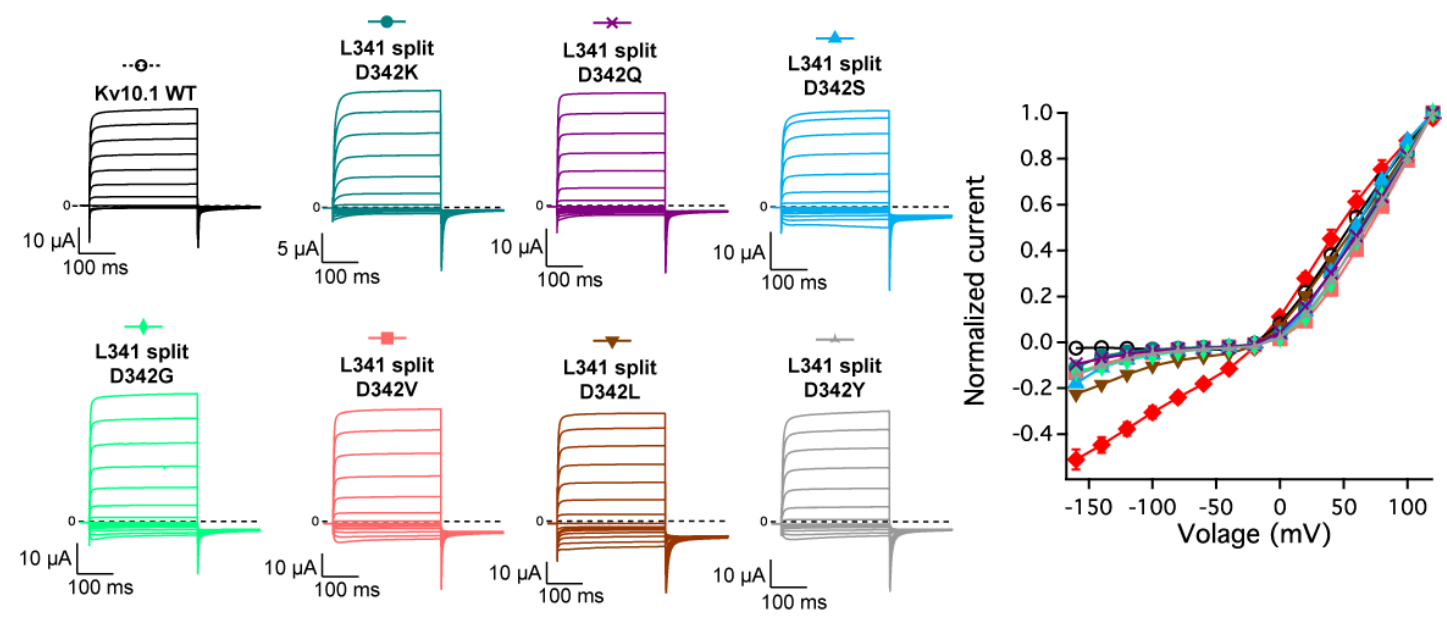

C
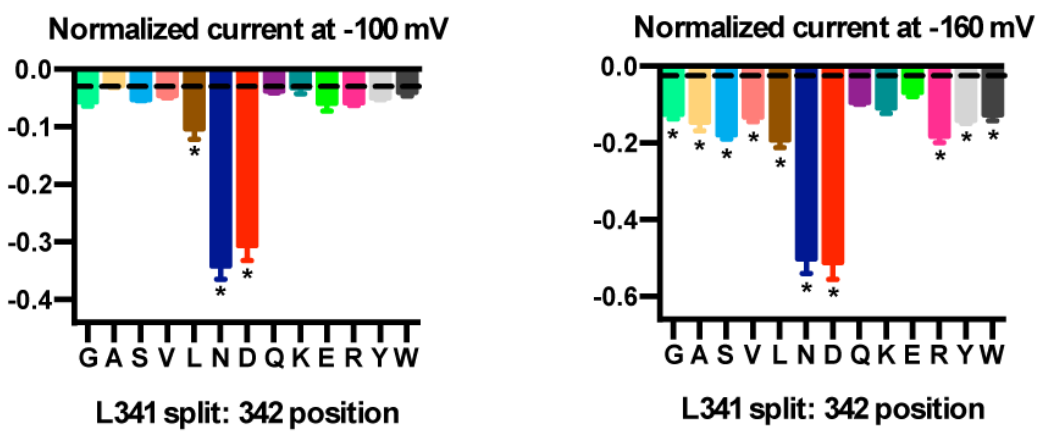

Figure 8. Restoration of wild-type-like closure with point mutations in S4-S5 linker. (a) Representative currents of the wild-type, L341 split, D342 mutants in L341 split and split channels carrying a deletion in S4-S5 linker. The voltage protocol is shown in upper right corner. (b) The corresponding normalized, average current values plotted against voltage $(n=7-18)$. Error bars denote SEM. Because the IV curves of most construct are overlapping, the data are divided into two graphs. WT and L341 split appear in both graphs as references. (c) Normalized current at $-100 \mathrm{mV}$ and $-160 \mathrm{mV}$ plotted against the residue at 342 position (mean \pm SEM). The order from left to right reflects residue size, the dashed line indicates the current level of the wild-type. 


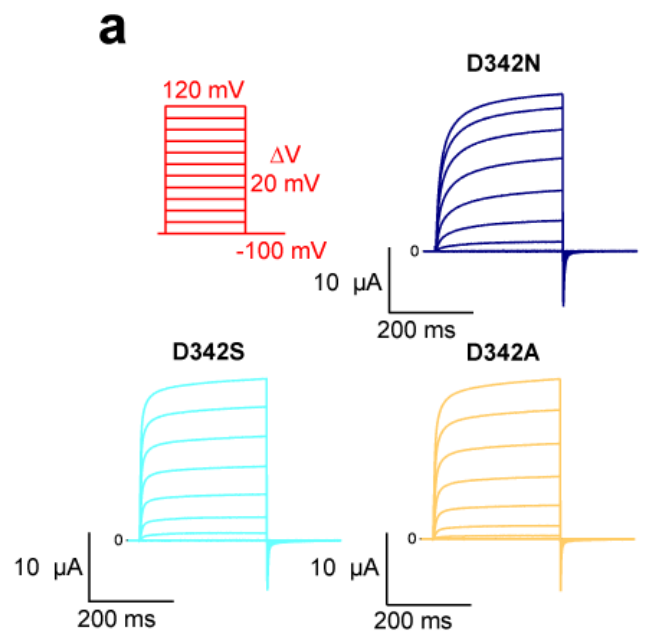

b

\section{C}
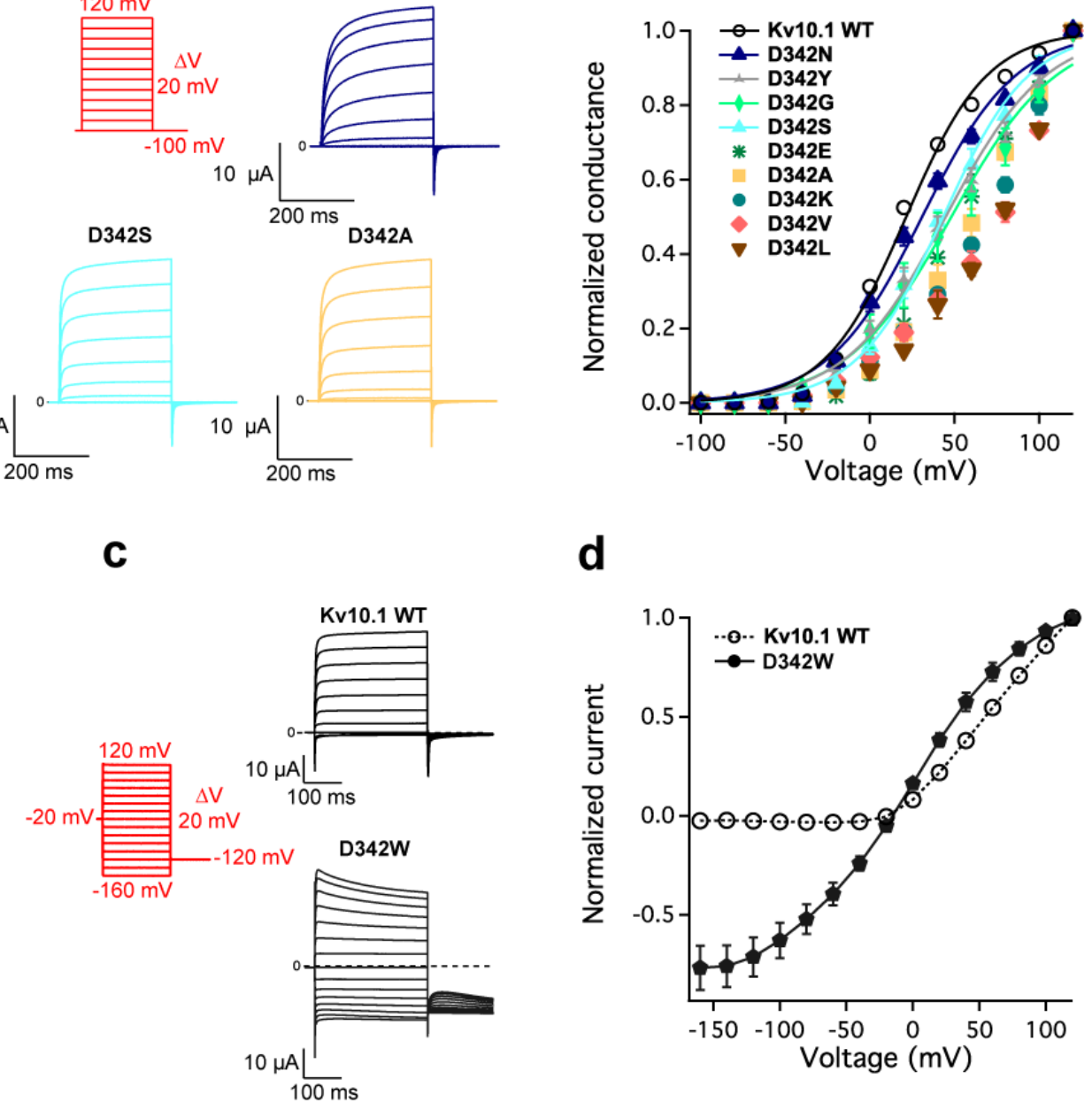

d

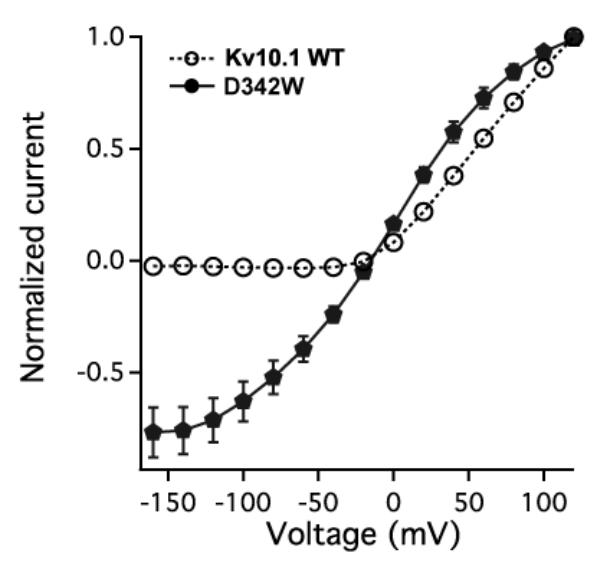

Figure 9. D342 mutants in the full-length channel. (a) Representative currents of selected D342 mutants recorded with the indicated voltage protocol (b) Normalized conductance plotted against voltage. Data points represent mean \pm SEM ( $\mathrm{n}=6-13)$, the curves are Boltzmann fits with the following Vhalf values: $21.4 \pm 1.7 \mathrm{mV}$ (WT), $30.8 \pm 2.0 \mathrm{mV}$ (D342N), $43.3 \pm 1.5 \mathrm{mV}$ (D342S), $44.8 \pm 2.0 \mathrm{mV}$ (D342Y), 48.1 $\pm 2.3 \mathrm{mV}$ (D342G). Fitting was not done for the other mutants, because the conductance did not saturate in the voltage range tested. (c) Representative currents of the wild-type and D342W mutant. (d) Normalized current plotted against voltage (mean \pm SEM, $n=10$-14).

We next checked if wild-type-like kinetics can be restored in Y347 split by mutating Y347. We were able to eliminate the super-slow deactivation component by mutations to Phe and Leu, which enabled complete closure within $500 \mathrm{~ms}$ (Fig. 10b). Notably, Y347F and Y347L mutants also had slower activation kinetics and biphasic GV curves (Fig. 10c-e). Since Phe did not mimic Tyr and the effects of Phe and Leu substitutions were almost indistinguishable, aromaticity of the side chain at the position 347 does not seem crucial. 
a

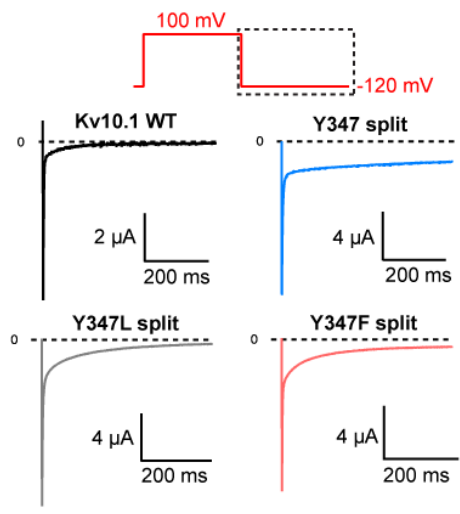

C
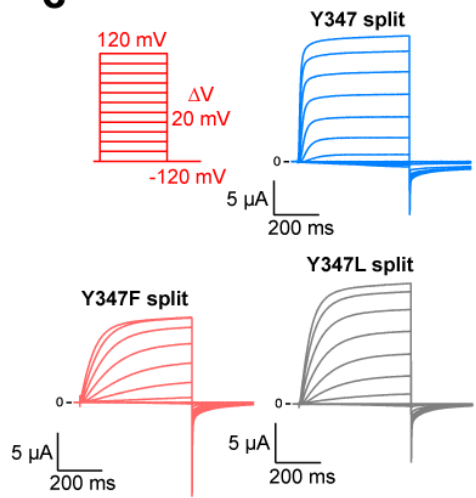

d

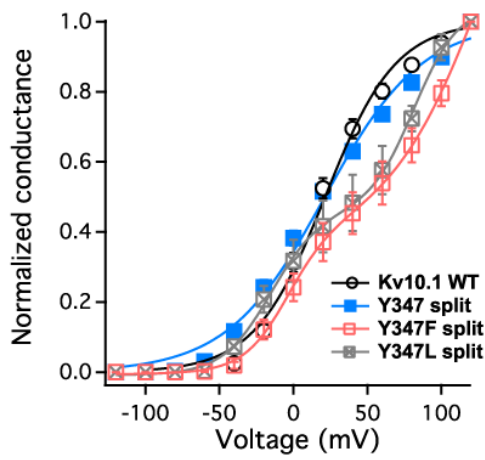

b

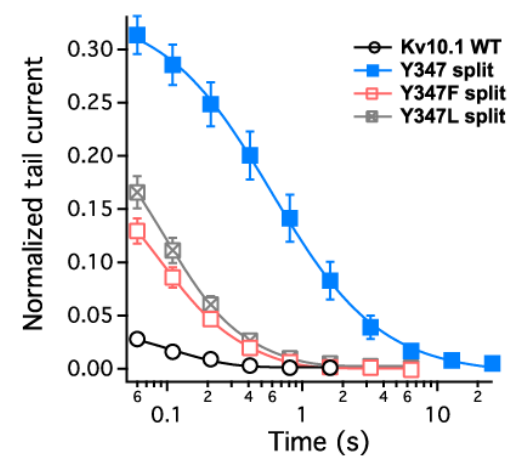

e



Figure 10. Y347 mutations restore deactivation kinetics of Y347 split.

(a) Representative tail currents of the wild-type, Y347 split and Y347 mutants in Y347 split. (b) Tail currents (normalized to the steady-state current at the end of the depolarizing segment of the voltage pulse) plotted against time at negative potential (mean \pm SEM; n=7). (c) Representative currents of Y347 split and Y347 mutants in response to the voltage protocol shown. (b) Normalized conductance values plotted against voltage. The data points represent mean \pm SEM $(n=7-8)$, the curves are single or double Boltzmann fits. (c) Time necessary for current to reach $90 \%$ of its maximal value plotted against the length of the interval at $-120 \mathrm{mV}$ in the reactivation protocol (mean $\pm \mathrm{SEM}$; $=7$ ).

This result points to the importance of tyrosine hydroxyl group, which is the only structural difference between Phe and Tyr, making Tyr less hydrophobic and enabling it to participate in hydrogen bonds. Apart from that, having a large, hydrophobic residue at this position might be important, as mutations to Ala and Ser considerably reduced functional expression.

Taken together, these findings suggest that even when S4-S5 linker is not continuous, important functional interactions of linker amino acids remain. Furthermore, it is truly striking that S4 can be disconnected from the linker with almost complete impunity if D342 is mutated to another amino acid. 


\section{Single molecule colocalization between fluorescently tagged VSD and PD}

To investigate the co-assembly between VSD and PD, we used the method of single-molecule counting/colocalization[71, 72]. In short, fluorescently tagged channels were imaged in the plasma membrane of Xenopus oocytes with a total internal reflection fluorescence (TIRF) microscope. This variant of microscopy allows to study membrane proteins without exciting fluorophores in the bulk of the cell.


Figure 11. Single molecule colocalization between fluorescently tagged VSD and PD. (a) Average colocalization coefficients \pm SEM, after subtraction of random colocalization ( $n=8-14)$. Tagged full-length subunits were used as positive control. (b-e) Representative $13 \times 13 \mu \mathrm{m}$ images for each condition and mean numbers of fluorescent spots per image \pm SEM.

Xenopus oocytes are a convenient expression system, because expression can easily be adjusted to a level, where single channels can be seen as diffractionlimited spots by varying cRNA concentration. We tagged VSD with EGFP at the N- 
terminus and PD with mCherry at the C-terminus, and determined their relative localization in the plasma membrane.

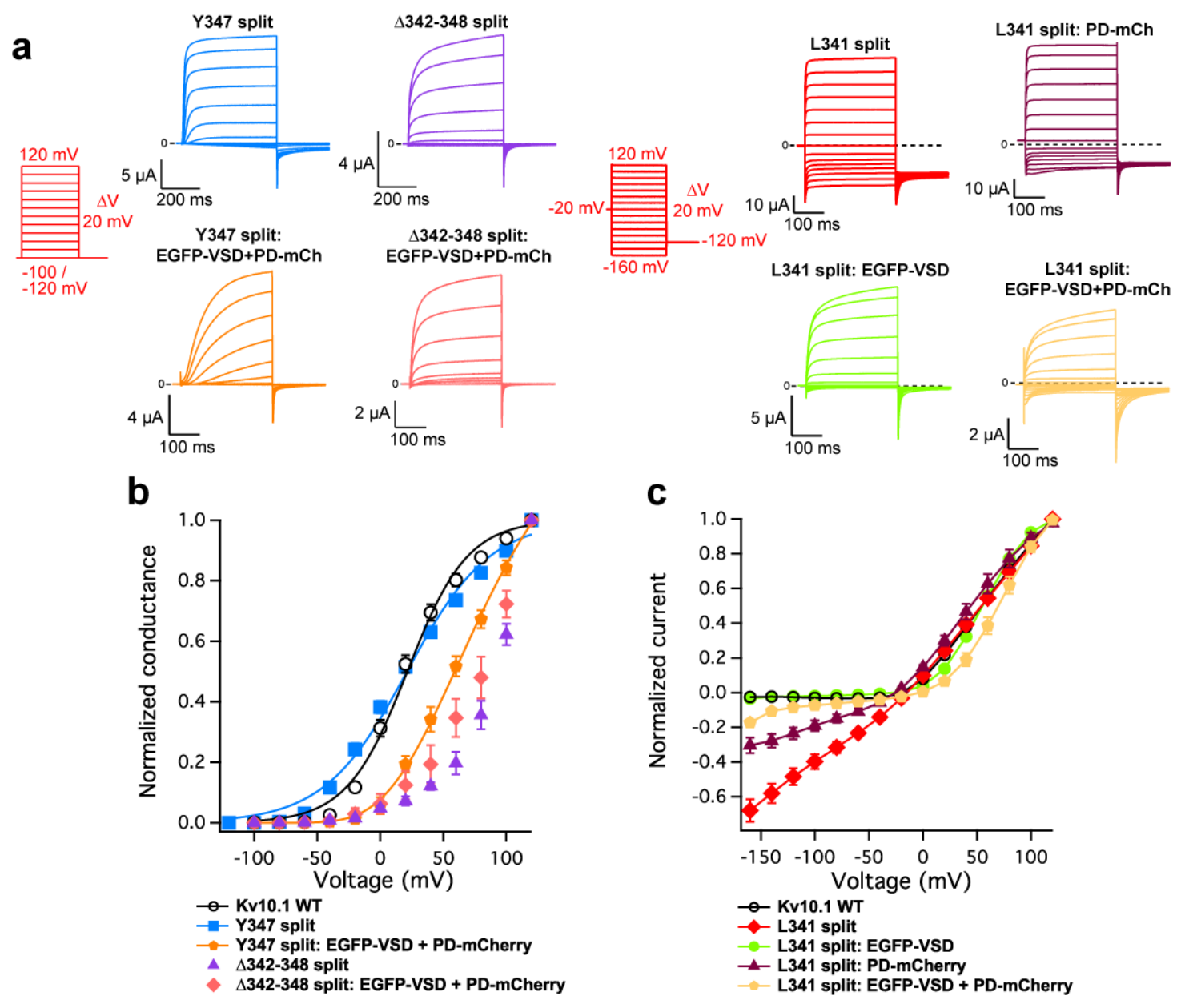

Figure 12. Split channels remain functional after tagging with fluorescent proteins. (a) Representative currents of the tagged and untagged split channels recorded with the indicated voltage protocols. We noticed that N-terminal EGFP fusion greatly facilitates closure of L341 split. (b) The corresponding average conductance-voltage ( $n=5-10)$ and (c) current-voltage curves (n=7-14). Error bars denote SEM.

When we tried to express EGFP-tagged VSD alone, it either did not express on the surface of the oocytes or expressed poorly and was moving fast (Supplementary Movie 1). By contrast, we observed stable diffraction-diffraction limited spots upon expression of mCherry-tagged PD (Supplementary Movie 2). Interestingly, we could observe stable spots for both VSD and PD, when they were co-expressed (Supplementary Movie 3). This finding suggests that co-assembly with PD is necessary for VSD to stably express in the plasma membrane. 
Since expression of VSD depended on the presence of PD, we expected higher colocalization between them than in the case of fluorescently tagged fulllength subunits (because EGFP or mCherry-tagged full-length subunits can also assemble with themselves, reducing the rate of colocalization between them). For colocalization experiments, we selected three split channels with very distinct biophysical properties: L341 split, Y347 split and $\Delta 342-348$ split and verified that they remained functional after tagging (Fig. 12). Interestingly, the N-terminal EGFP fusion restored the closure of L341 split, hinting at the possibility of interaction between D342 and N-terminal cytoplasmic domains.

We imaged 50-250 fluorescent puncta per patch in 8-14 13x13 $\mu \mathrm{m}$ optical patches. After subtraction of random colocalization, we got a very similar colocalization coefficient for all three split channels tested $(48 \pm 2 \%, 48 \pm 4 \%$, $45 \pm 3 \%$, respectively; mean $\pm S E M$ ), and found it to be significantly higher, compared to the full-length subunits $\left(30 \pm 2 \%\right.$, mean \pm SEM; $\mathrm{p}<1 \times 10^{-3}$ for L341 and Y347 split and $\mathrm{p}<1 \times 10^{-2}$, Bonferroni's multiple comparison test) (Fig. 11a). We also noted that when the tagged full-length subunits were coexpressed, we observed many more non-colocalized EGFP than mCherry puncta, owing to faster maturation of EGFP (Fig. 11b). Remarkably, this ratio was reversed, with very few non-colocalized EGFP puncta, if the covalent bond between VSD and PD was broken (Fig. 11c-e), again indicating that the EGFP-tagged VSD needs to interact with PD to be in the plasma membrane. These results show that VSD and PD coassemble even if they are not covalently bound. A further implication is that different biophysical properties of split channels do not seem to arise from different VSD-PD assembly.

\section{Breaks in S4-S5 linker affect VSD motion and coupling with PD}

Constitutive activity of specific split channels could result from decoupling between VSD and PD. It could also be caused by a bias towards the activated conformation of the VSD if coupling between them is preserved in the absence of a covalent link. To distinguish between these options, we measured voltagedependency of VSD activation reported by VCF. We used I319C mutants labeled 
with TMRM, after verifying I319C mutation by itself has only limited consequences for the voltage-dependency of the channel (Fig. 13).
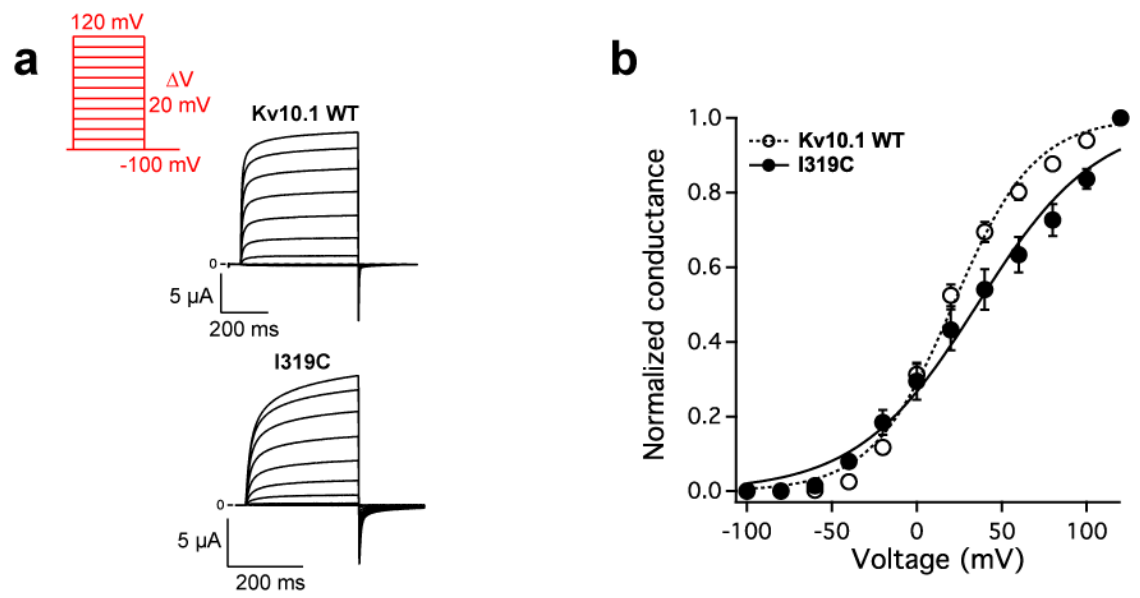

Figure 13. I319C mutation has only limited functional consequences. (a) Representative currents of the wild-type and I319C mutant recorded with the indicated voltage protocol. (b) Normalized conductance plotted against voltage. Data points represent mean \pm SEM $(n=10-11)$, the curves are Boltzmann fits. The

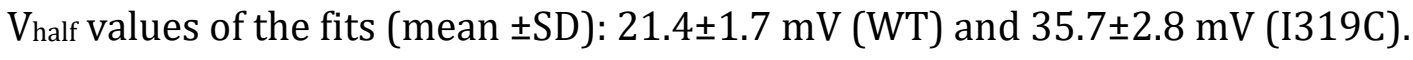

As in the colocalization experiments, we focused on three split channels which cover the whole spectrum of GV curves: left-shifted, constitutively active L341 split, right-shifted $\Delta 342-348$ split, and Y347 split with no significant shift, compared to the wild-type (Fig. 14a, b). Although magnitudes of VCF signals are not straightforward to compare, as every oocyte is recorded with a slightly different gain of the photomultiplier, we noted that split constructs showed lower voltage-dependent changes of fluorescence than the full-length channel, probably owing to a difference in expression levels. Among the split channels, L341 split consistently gave the lowest signal (Fig. 14c). Surprisingly, the midpoint of fluorescence-voltage (FV) curves was nearly overlapping between the full-length channel (Vhalf $-57.7 \pm 3.0 \mathrm{mV}$; mean \pm SD) and L341 split ( $V_{\text {half }}-54.3 \pm 2.8 \mathrm{mV}$ ) (Fig. 14d). Y347 split and $\Delta 342-348$ split showed modest positive shifts of 10 and 20 $\mathrm{mV}$, respectively ( $\mathrm{V}_{\text {half }}-49.0 \pm 3.6 \mathrm{mV}$ and $-35.6 \pm 3.4 \mathrm{mV}$; the intervals encompassing the mean \pm 3 SD are overlapping with the full-length channel for Y347 split and non-overlapping for $\Delta 342-348$ split). Interestingly, the separation of GV and FV curves became smaller in Y347 split and larger in $\Delta 342$-348 split, with respect to the full-length channel (exact estimation is impossible for $\Delta 342$ - 
348 split, because the GV curve did not saturate in the voltage range tested). If this holds true, different breaks in S4-S5 linker can influence coupling between VSD and PD in opposite ways.



Figure 14. Voltage-dependency of conductance and VSD activation of specific split channels. (a) Representative currents of the wild-type, L341 split, Y347 split and $4342-348$ split recorded with the indicated voltage protocol, with the exception of L341 split where the holding potential was $-20 \mathrm{mV}$. (b) Normalized conductance (mean \pm SEM; $n=7-10$ ) plotted against voltage, the curves are Boltzmann fits with the following Vhalf values (mean \pm SD): $-28.0 \pm 1.8 \mathrm{mV}$ (L341 split), $21.4 \pm 1.7 \mathrm{mV}$ (WT), $21.7 \pm 2.1 \mathrm{mV}$ (Y347 split). Fitting was not done for $\Delta 342-348$ split, because the conductance did not saturate in the voltage range tested. (c) Representative voltage-dependent fluorescence changes reported by I319C-TMRM. Only traces elicited by the voltage-steps of $-160 \mathrm{mV}$ (blue), $-60 \mathrm{mV}$ (green) and $60 \mathrm{mV}$ (red) are shown. (d) Normalized changes of fluorescence plotted against voltage (mean \pm SEM; $n=5-10$ ), the curves are Boltzmann fits. Inset contains the $V_{\text {half }}$ values of the Boltzmann fits (mean \pm SD): $-54.3 \pm 2.8 \mathrm{mV}$ (L341 split), $-57.7 \pm 3.0 \mathrm{mV}$ (WT), $-49.0 \pm 3.6 \mathrm{mV}$ (Y347 split), $-35.6 \pm 3.4 \mathrm{mV}(\Delta 342-348$ split).

The VCF experiments also posed an apparent contradiction, because the FV curve of L341 split did not have a negative shift, despite its inability to fully close. This could indicate that VSD and PD are decoupled, and VSD motion no longer leads to effective channel closure. Since VCF reports local protein motions around the fluorophore, it is also possible that the resting conformation of S4 is affected 
in L341 split. We checked if the activation kinetics of L341 split are slowed down by $\mathrm{Mg}^{2+}$, which stabilizes the deactivated conformation of the VSD[51]. Strikingly, the presence of $\mathrm{Mg}^{2+}$ had almost no effect (Fig. 15).



b
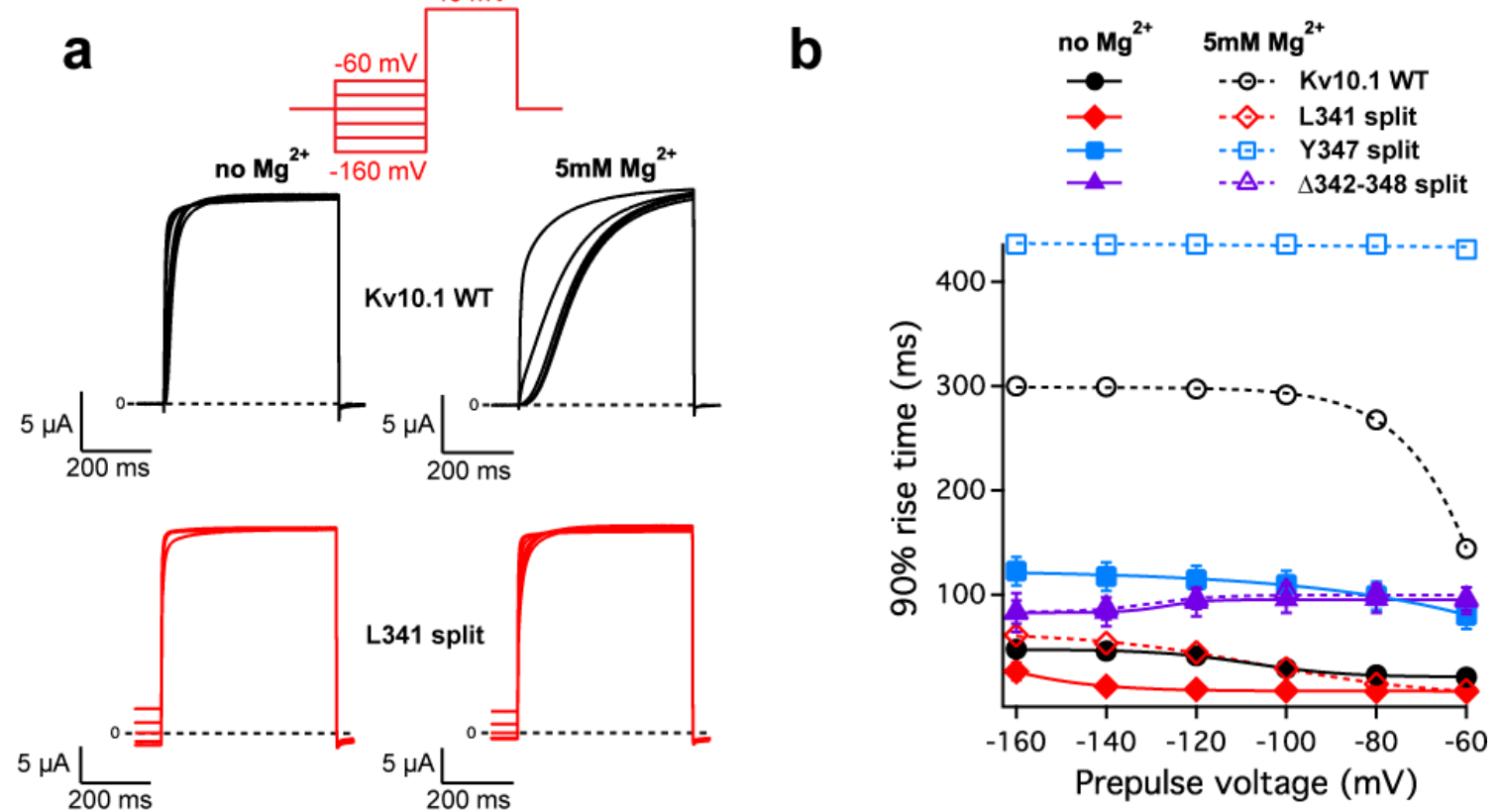

C
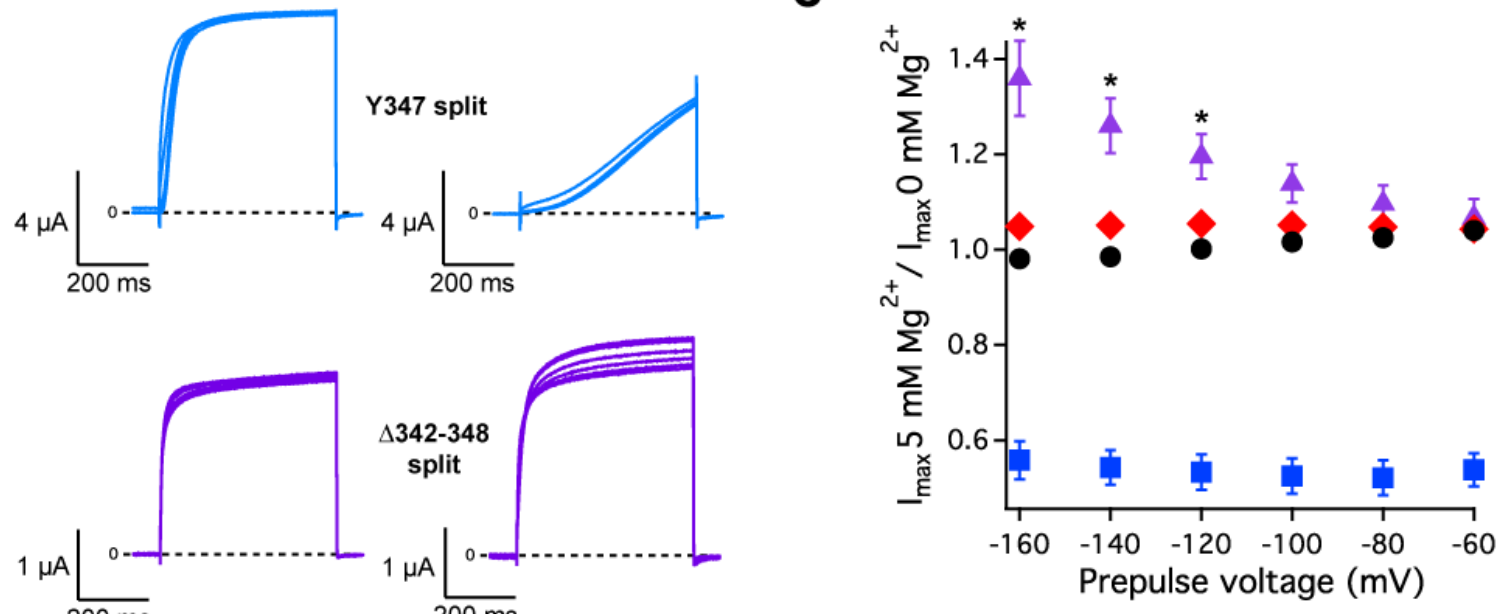

Figure 15. Effect of $\mathbf{M g 2}^{+}$on current activation kinetics. (a) Representative currents of the wild-type, L341 split, Y347 split and $\Delta 342-348$ split elicited with the indicated voltage protocol, with (left) and without (right) $5 \mathrm{mM} \mathrm{Mg}^{2+}$ in the extracellular medium. In the voltage protocol, the duration of the conditioning prepulse was 5s. (b) Time needed for current to reach $90 \%$ of the maximal value plotted against the prepulse voltage (mean \pm SEM; $n=7-10$ ) (c) The ratio of peak currents with and without $\mathrm{Mg}^{2+}$ in the extracellular medium (mean $\pm \mathrm{SEM} \mathrm{n=7-10)}$.

However, just the loss of $\mathrm{Mg}^{2+}$ effect is insufficient to conclude that the resting position of S4 is different, because if VSD and PD are decoupled $\mathrm{Mg}^{2+}$ binding is 
also expected to have no or little effect, depending on the extent of decoupling. Thus, more experiments are needed to determine the resting position of S4 in L341 split.

We also checked how $\mathrm{Mg}^{2+}$ affects the other split channels. Slowing-down of activation kinetics due to $\mathrm{Mg}^{2+}$ was greatly augmented in Y347 split, compared to the full-length channel. Interestingly, in $\Delta 342-348$ split the activation kinetics were unaffected by $\mathrm{Mg}^{2+}$, but we observed significant increases of the peak current for the most hyperpolarizing prepulses with $\mathrm{Mg}^{2+}\left(\mathrm{p}<1 \times 10^{-4}\right.$ for -160 and $-140 \mathrm{mV}$, $\mathrm{p}<1 \times 10^{-3}$ for $-120 \mathrm{mV}$, Bonferroni's multiple comparisons test), almost as if $\mathrm{Mg}^{2+}$ binding caused conformational changes in the VSD that enhance interaction with PD upon depolarization. This observation might support the idea that the coupling between VSD is compromised in the absence of two helical turns in the linker, and the probability that VSD activation will lead to channel opening is lower than in the full-length protein or in the split channels which do not have a deletion (note that we have two different decoupling scenarios: VSD is decoupled from PD in L341 split, because it cannot close, or $\Delta 342-348$ split, because it opens less effectively).

\section{Isolated pore module prefers the closed conformation}

To further investigate the interaction between VSD and PD, we took advantage of the fact that in Kv10.1 S6 helix lacks the PVP kink, which has been postulated to act as a pivot important for gating, and contains no other helix-breakers. Following a study in Kv11.1[73], we speculated that introducing a proline at some positions in S6 would lock the channel in the open state by forcing a break in S6. Indeed, we found that mutating Q476 and Q477 to a proline results in constitutively active full-length channels with robust currents (Fig. 16). Interestingly, Q476P mutation caused a strong inward rectification, whereas Q477P mutant had a nearly linear dependence of current on voltage. Given our observation of PD complexes without VSD at the single-molecule level, we next tested if proline substitutions in S6 have a similar effect on PD expressed alone. Both Q476P and Q477P mutations failed to give rise to $\mathrm{K}^{+}$currents, when expressing the isolated pore domain (Fig. 17, Fig. $18 \mathrm{a}, \mathrm{b})$. 
a
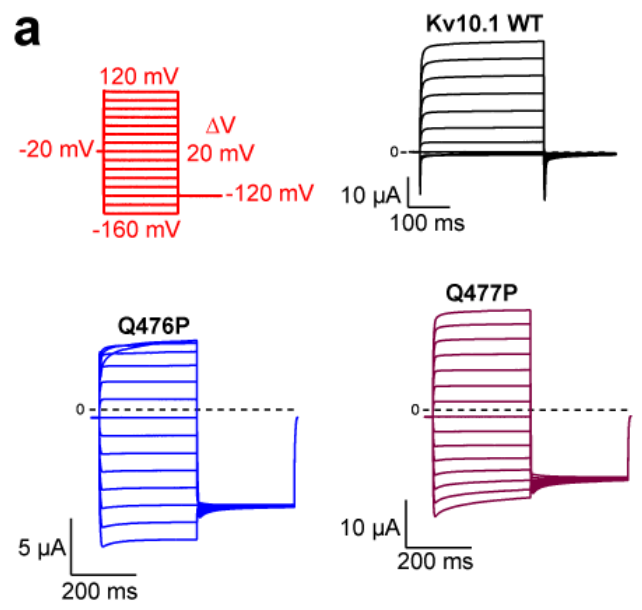

b

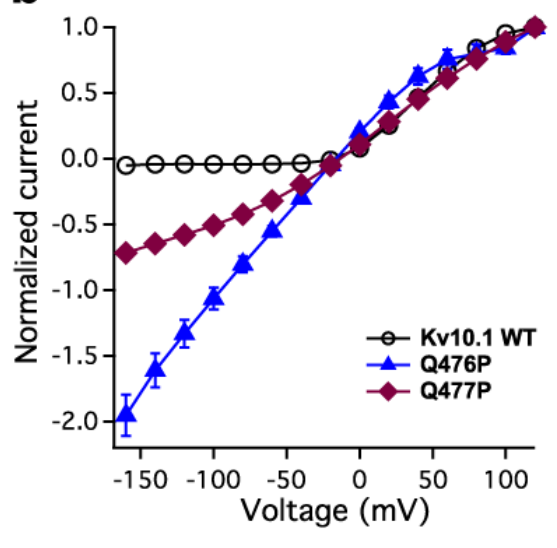

Figure 16. Proline substitutions in inner S6 lock the full-length channel in the open state. (a) Representative currents of the wild-type, Q476P mutant and Q477P mutant recorded with the indicated voltage protocol. (b) Normalized, average current values plotted against voltage $(n=5-11)$. Error bars denote SEM.

a

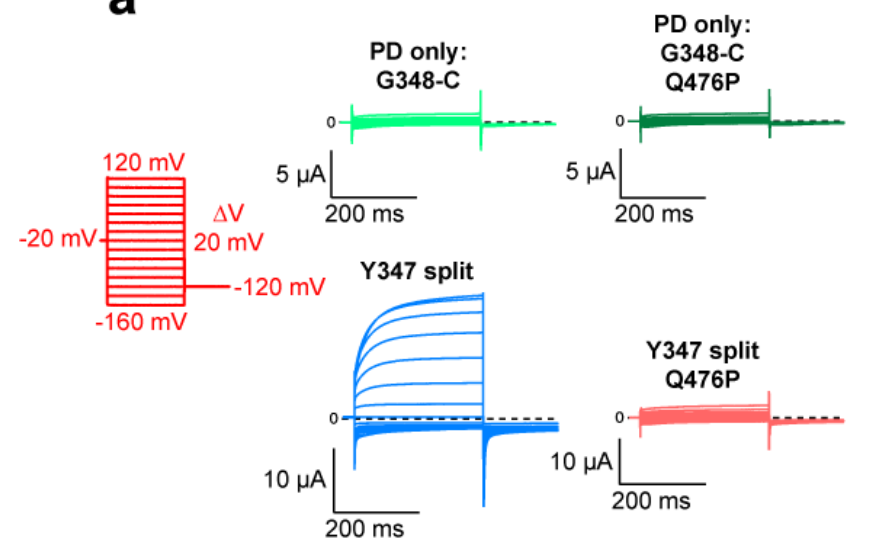

b



Figure 17. Effects of Q476P mutation on PD expressed in isolation and with the VSD. (a) Representative currents from oocytes expressing the isolated pore domain (G348-C stands for the PD sequence starting from Gly348 and ending at the C-terminus), Q476P mutant in the isolated pore domain, and Y347 split, with and without $\mathrm{Q} 476 \mathrm{P}$ mutation. (b) The corresponding average current values \pm SEM $(n=9-10)$ plotted against voltage (without normalization).

Upon co-expression with VSD, Q476P mutant had no current (Fig. 17), and Q477P mutant was constitutively active (Fig. $18 \mathrm{a}, \mathrm{b}$ ), similarly to the full-length channel. We verified that the isolated pore with Q477P mutation is expressed in the plasma membrane by tagging it with EGFP at the C-terminus and imaging with a TIRF microscope (Supplementary Movie 4). Taken together, these findings suggest that even if access to the pore is granted by a break in S6, the presence 
of the VSD is still required for permeation, which is a bit surprising given the results obtained with sensorless pores of specific bacterial channels $[56-58,61]$.

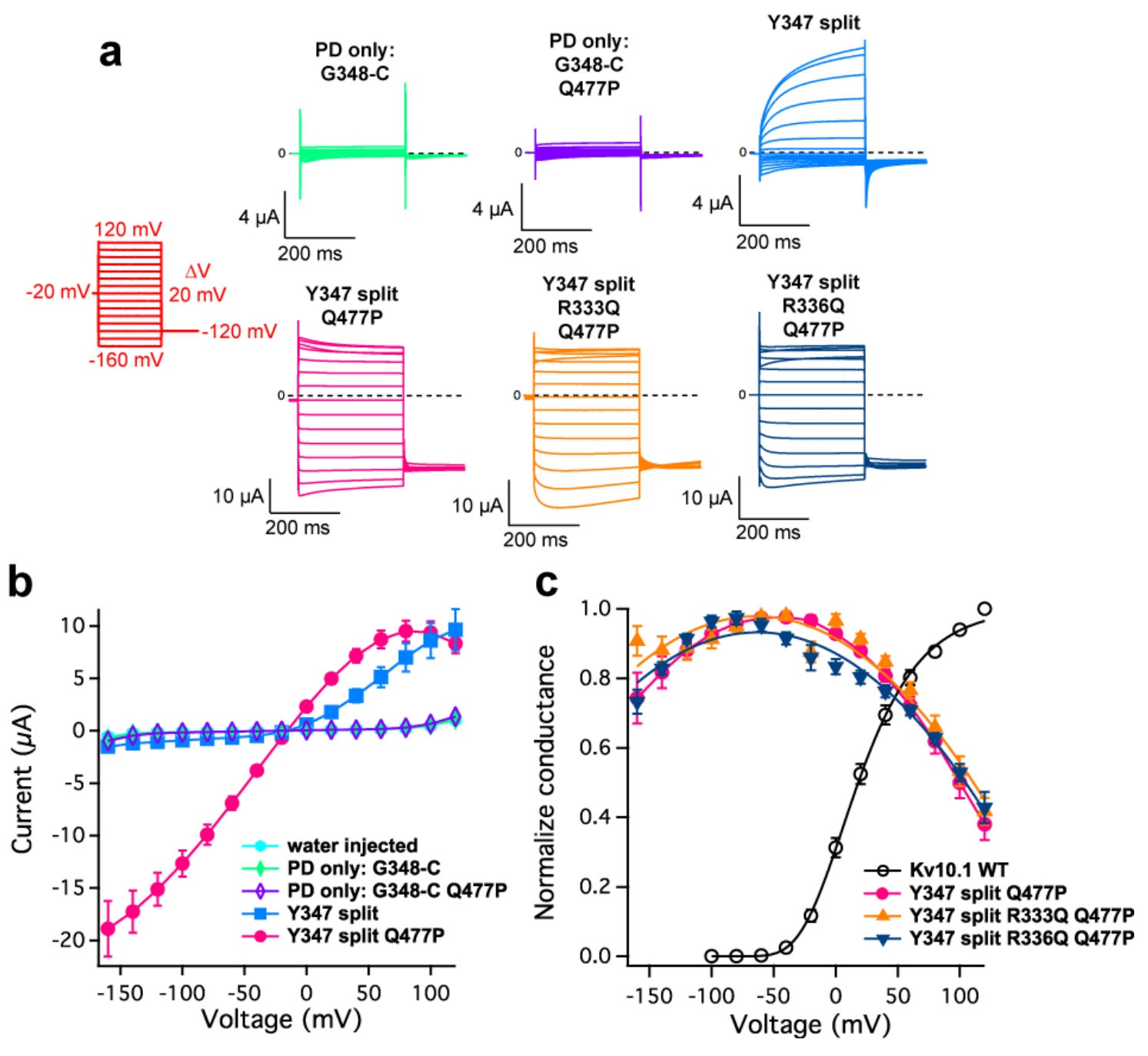

Figure 18. Effects of Q477P mutation on PD expressed in isolation and with the VSD. (a) Representative currents from oocytes expressing the isolated pore domain (G348-C stands for the PD sequence starting from Gly348 and ending at the C-terminus), Q477P mutant in the isolated pore domain, and Y347 split, with or without Q477P mutation or additional VSD mutations. (b) The corresponding average current values \pm SEM plotted against voltage $(n=7-10$; without normalization). (c) Normalized conductance values plotted against voltage. The data represent mean \pm SEM $(n=6-10)$, the curves are parabola fits for Q477P mutants and a Boltzmann fit for the wild-type.

We then introduced R333Q and R336Q mutations in the VSD that shift voltage-dependency of the channel to positive and negative values, respectively (Fig. 19). Remarkably, although the presence of VSD was essential for the constitutive activity of split channels with Q477P mutation, the shifts of voltage- 
dependency mattered little, as all three resulting split channels behaved almost equally (Fig. 18a, c). Unexpectedly, they showed strong rectification, with the open probability decreasing at positive potentials, similarly to Q476P mutation in the full-length channel. This might suggest that the position of $\mathrm{S} 6$ residues is slightly different in the split channel or reveal a degree of intrinsic voltage-sensitivity of the pore.
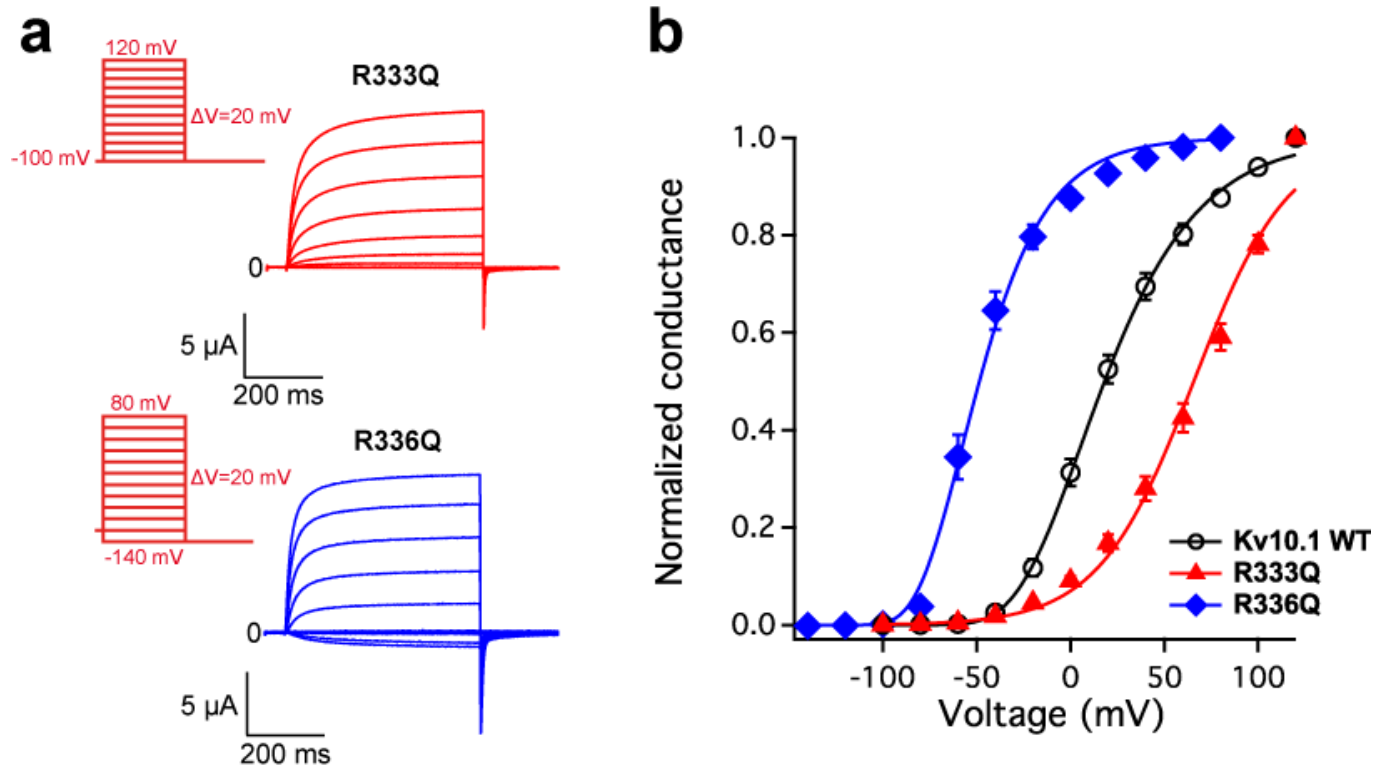

Figure 19. Charge neutralizations in S4 can shift voltage-dependency of the channel to both positive and negative potentials. (a) Representative currents of R333Q and R336Q mutants recorded with the indicated voltage protocols. (b) Normalized conductance plotted against voltage. Data points represent mean \pm SEM ( $n=9-10)$, the curves are Boltzmann fits.

From a broader perspective, these results could point to a dual role of the VSD, which might be structurally important for the permeation pathway to work, apart from switching between resting to active states to regulate the conformation of the pore. Also, if the pore in isolation prefers the closed conformation, it is more likely that we see decoupling between VSD and PD in $\Delta 342-348$ split, compared to L341 split which cannot fully close. 


\section{Discussion}

In this dissertation, we show several important findings on voltage-gating in the absence of a covalent link between VSD and PD. First, if a break is introduced in S4-S5 linker on the side of S4, the resulting split channels are constitutively active. Second, specific breaks closer to S5 lead to dramatic alterations of channel kinetics. Third, point mutations of crucial linker amino acids almost completely abolish the constitutive activity and restore wild-type-like deactivation kinetics of specific split channels. Remarkably, if D342 is mutated to another amino acid, except for Asn, VSD can be disconnected from the S4-S4 linker and PD with almost complete impunity. Fourth, fluorescently-tagged VSDs and PDs colocalize in the plasma membrane at the single molecule level. Fifth, disconnection between VSD and PD affects VSD motion, although these effects seem subtler than the changes of conductance. Finally, the presence of VSD might be structurally important for the permeation pathway to be functional. Below we discuss these results in the context of the current understanding of voltage-gating.

\section{Constitutively active split channels}

As mentioned above, constitutive activity of specific split channels could arise from decoupling between VSD and PD (if the isolated pore prefers the open state) or trapping of VSD in the active conformation. At this point, we are unable to distinguish between these options. However, other lines of evidence point to the intrinsic preference of the pore for the closed conformation, as discussed below. To further investigate the resting conformation of the VSD of constitutively active channels, we should employ more tools. One possibility would be to do a MTS accessibility assay. If the resting conformation of the VSD is not the same as in the full-length channel, we expect to see changes in the voltage-dependency of accessibility of sites in the S3-S4 loop to MTS reagents. On the contrary, if the accessibility pattern remains the same, the resting position of the VSD, as well as its voltage-dependent conformational changes are likely to be similar.

It is also worthwhile to consider the results of D342 mutagenesis in L341 split. Since only the isosteric Asn could mimic Asp, it is likely that carbonyl groups 
of their side chains are acceptors in a hydrogen bond stabilizing the open conformation of the channel. No other form of interaction seems compatible with the observation that all the other residues tested produce a similar outcome, irrespectively of their physicochemical properties. If the interaction were electrostatic or steric, we should have seen a correlation of the channel behavior with the size or the charge of the substituted side chain, which was not the case. Additional evidence for the conclusion that Asp and Asn at the position 342 participate in an interaction which energetically favors the open state comes from positive GV shifts of D342 mutants in the full-length channel. Interestingly, the homologous D540 residue in closely related Kv11.1 participates in an electrostatic interaction stabilizing the closed state ${ }^{[40,41]}$. This would imply that despite very high homology between Kv10.1 and Kv11.1, equivalent residues make a very distinct contribution to gating mechanisms. Moreover, in Kv11.1, D540K mutation made the channel reopen at hyperpolarizing voltages, whereas D342K mutation in Kv10.1 did not have such effect. Next, we should ask where the interacting partner of D342 is. A serious candidate is Q476 at the channel gate, whose counterpart in Kv11.1 is R665, the interaction partner of D540. It also cannot be excluded that D342 interacts with other parts of the gating machinery. Cytoplasmic domains have been implicated in voltage-gating of KCNH channels ${ }^{[18]}$. Two lines of evidence point to the possibility that D342 has an interaction partner in the N-terminal PAS domain. First, the N-terminal EGFP fusion restored the closure of L341 split. This could occur if fluorescent tagging at the N-terminus could slightly alter the position or the structure of the PAS domain in a way that the interaction with D342 is no longer favourable. Second, in Kv11.1, the Nterminal tail of the PAS domain and the N-terminal portion of S4-S5 linker lie within atomic distances ${ }^{[74,75]}$. However, more evidence is needed to confirm or rule out an interaction of D342 with the PAS domain.

Finally, in light of the finding that the closure of L341 split can be restored with point mutations of D342, it is unlikely that S4 and S4-S5 linker have to move together as a rigid body to gate the channel. As far as breaks closer to the S5 helix are concerned, one could still make the argument that a substantial portion of the linker still undergoes a concerted motion with S4. Flexibility between the linker and S5 might be needed to effectively transfer energy to the pore module ${ }^{[37]}$. 
In the case of L341 split, however, the break is introduced one residue after the last voltage-sensing arginine, so virtually the entire S4-S5 linker is disconnected from S4. It is thus extremely surprising that D342 mutants in L341 split behaved so similarly to the full-length channel. This result might suggest that we are still quite far from mechanistic understanding of electromechanical coupling, because none of the available gating models would predict such an outcome. It might also mean that we should look for interactions between VSD and PD that are not mediated by the S4-S5 linker. For instance, the contact area between S1 and S5 could provide a structural platform for such interactions. Also the interaction between the PAS domain at the N-terminus and the CNBHD at the $\mathrm{C}$-terminus is potentially relevant for the function of split Kv10.1 channels.

\section{Changes of activation and deactivation kinetics}

The observed alterations of kinetics in specific split channels suggest that discontinuity between S4 and S5 can not only change the free energies associated with the open and the closed conformation, as seen in constitutively active split channels, but also influence the height of energy barrier between them. Alternatively, in the case of Y347 split, some shallow closed states of the channel protein might turn into open states, without changing transition rates between them. Thus, a possible explanation for the super-slow deactivation component is that the split channel visits conformations corresponding to shallow closed states in the full-length channel, which now have some conductance. Because the onset of slower activation measured with the reactivation protocol (Fig. 6b) correlates with the super-slow closure (Fig 5a), it is tempting to assume that only deeper closed states allow effective closure of Y347 split. This explanation is also consistent with the observation that VSD deactivation time constants are overlapping between Y347 split and the full length channel (Fig. 7). Besides, it is worth pointing out that although the FV curve of Y347 split is slightly right-shifted, compared to the full-length channel, Y347 split has more conductance at -60 and $-40 \mathrm{mV}$ (and no overall GV shift). If shallow closed states are indeed converted into open states, a closer investigation of Y347 may lead to further insights into the molecular determinants of electromechanical coupling. To our knowledge, this 
would be the first case of a perturbation of a voltage-gated $\mathrm{K}^{+}$channel which turns closed states into open states.

The slower activation kinetics of Y347 split can be at least partly attributed to slower VSD motion (Fig. 7). The question remains if G348 split, which activates even slower than Y347 split, also shows slower VSD motion. If so, we should also ask why these particular breaks in S4-S5 linkers delay the outward motion of S4, while others probably do not (based on kinetics of current activation).

\section{VCF measurements of VSD motion}

Probing VSD motion led us to two important conclusions. First, since we observed changes of both voltage-dependency and kinetics of VSD motion, the interaction between the VSD and PD is a two-way street. Interestingly, out of three split channels tested, two showed positive FV shifts. Second, we observed both increased ( $\Delta 342-348$ split) and decreased (Y347 split) separation between GV and FV curves, suggesting different breaks in S4-S5 linker affect coupling between VSD and PD in opposite ways. As mentioned in the Introduction, increased separation of GV and FV curves does not necessarily indicate that VSD and PD are decoupled, because coupling between them might be positive or negative ${ }^{[55]}$. However, if we also consider that we have removed a huge part of the coupling interface of $\Delta 342$ 348 split, it is reasonable to expect that coupling between VSD and PD will be reduced. If strain from the S4-S5 linker were to maintain the channel closed at negative potentials (in other words if coupling between VSD and PD were negative), deleting a huge part of the linker should result in difficulties with channel closure and a preference for the open state. On the contrary, in the case of positive coupling, $\Delta 342-348$ split should prefer the closed state, as it did in our experiments. Thus, our results suggest positive coupling between VSD and PD.

In this study, we just included VCF data from one labelling site. As a future direction, we should include more labelling sites and compare their readouts (some caution is necessary, because the resting position of S4 might be different in split channels, affecting VCF results). In Shaker, when all VSDs have been activated channel opening requires a weakly voltage-dependent cooperative step, which can be separated from voltage-sensing motions by specific mutations in 
S4[45] and involves an additional gating motion of S4[47]. It would be of great interest to check if this additional component of S4 motion can be isolated in $\Delta 342$ 348 split at more positive voltages in the range of channel activation (the maximal depolarizing step in VCF experiments in Fig. 8 was $+60 \mathrm{mV}$, with the VSD being fully activated).

\section{Intrinsic preference of the pore and a dual role of the VSD}

A novel idea that comes from our experiments is that the presence of VSD might be structurally important for the permeation pathway to work, as shown by the experiments which used proline substitutions in inner S6. Unless it is coexpressed with VSD, the isolated pore of Kv10.1 does not conduct, even if a break in S6 is forced with a proline mutation that locks the full-length channel in the open state. It seems to follow that not only does the isolated prefer the closed conformation, but also that it is unable to adopt the conducting conformation without the VSD. It is also striking that VSDs with different voltage-dependencies can be used in the background of Q477P mutation with the same effect, suggesting that the contribution of VSD is not limited to switching between the resting and the active conformation. Here it is important to point out that if the pore can open without the VSD, but the open probability is low, we might have missed that in two-electrode voltage-clamp recordings because of endogenous currents in oocytes. Protein purification and reconstitution in lipid bilayers are a more elegant way to probe if the isolated PD can adopt the open conformation. With that said, our results support the MD simulations which predicted an intrinsic preference of the pore for the closed state in eukaryotic channels ${ }^{[43,44]}$, whereas isolated pore modules of voltage-gated bacterial channels seem to prefer the open state ${ }^{[56,58-61]}$.

\section{Split channels from other families}

Another key issue, which is a bit outside the scope of this dissertation, is whether a break in S4-S5 linker can be tolerated in other channel families. In our hands, split constructs of Kv1.4 and KCNQ channels showed no functional expression[70]. However, we did not test whether they assemble and show up in the plasma membrane. It is tempting to speculate that some split channels might assemble, 
and sit in the membrane as non-functional complexes, with VSD motion completely decoupled from the gate. Single-molecule imaging and VCF are perfect tools to address that. Alternatively, if the other channels we checked show no functional expression because they fail to assemble, the question if they would remain voltage-gated with a break in S4-S5 linker is still open. If the latter were the case, it would be extremely interesting to know if their assembly and trafficking can be enhanced by engineering the channel protein.

\section{Concluding remarks}

To recapitulate: although our results definitely increase the understanding of split Kv10.1 channels, we are not in a position to propose a coherent mechanistic model of voltage-gating in the absence of a covalent link between VSD and PD. Beyond doubt, our findings point to the importance of non-covalent interactions. Let us consider that in the channel structure various non-convalent interactions are possible between the parts of the gating machinery. Some of them are energetically favorable for the open and the closed conformation, whereas others affect the energy barrier between them. VSD motion and the resulting position of S4-S5 linker would change the energy landscape of the channel protein, putting weights on these interactions. In this scenario, the open probability and channel kinetics at any given voltage would be determined by the weighted mean of noncovalent interactions between the parts of the gating machinery. For small transmembrane voltage to be an effective switch, the open and the closed state cannot be very well separated energetically. Thus, changes of non-covalent interactions seem a very plausible mechanism for the channel to open and close. The estimates of energy associated with opening in Shaker fall roughly within the range of changing one non-covalent interaction per subunit. Moreover, functional, voltage dependent channels are evidence that these interactions remain even if VSD and PD are not covalently bound. Here we have shown that D342 and Y347 are crucial parts of the gating machinery in Kv10.1. To close down on the gating mechanism, it is necessary to find their interaction partners and identify other relevant interactions and their structural constraints. It would be extremely 
satisfying to combine functional experiments in split channels with structural data. 


\section{Materials and methods}

\section{Mutagenesis and expression in Xenopus oocytes}

The first split channels were generated from Kv10.1 sequence in the pSGEM oocyte expression vector by Dr. E. Lörinczi and Dr. J.C. Gomez-Posada, as previously described[70]. In short, two different Stop-X-Start cassettes were introduced at the desired position between VSD and PD, where $\mathrm{X}$ is a unique BamHI or EagI restriction site. BamHI and EagI flank the channel open reading frame (ORF), so once one of the cassettes was introduced, the corresponding enzyme could be used to excise the fragment coding for VSD or PD. The vector was then religated. From that point, VSDs and PDs of the subsequent split channels were made by removing or inserting codons from the parent split channel sequences by PCR-based mutagenesis. All site-directed mutagenesis was performed with the Quik Change Kit (Agilent Technologies), following manufacturer's instructions. For colocalization experiments, the relevant constructs were amplified by PCR and cloned in the multicloning site of pGEMHE$\mathrm{X}$-EGFP vector. N-terminus of the channel was connected to the $\mathrm{C}$-terminus of EGFP with the following 19-amino acid linker: GGSGGSGGSGGSGGRSRSS. mCherry fusions were generated with the In-Fusion kit (Clontech) by first cloning the relevant ORFs at the multicloning site of pmCherry-N1 vector (Clontech) and subcloning of the resulting fusion proteins into the oocyte expression vector pSGEM. C-terminus of the channel was connected to the N-terminus of mCherry with 6-amino acid linker: GGSGGS.

DNA constructs were linearized with SfiI or NheI and cRNAs were synthesized using T7 mMessage mMachine kit (Ambion). For the full-length constructs, oocytes were typically injected with 0.05-0.5 ng of RNA. For the split channels, more RNA was injected, typically 5-10 ng of each part. Before experiments, oocytes were kept at $18{ }^{\circ} \mathrm{C}$ in ND-96 medium (in mM: $96 \mathrm{NaCl}, 2 \mathrm{KCl}$, $0.2 \mathrm{CaCl}_{2}, 2 \mathrm{MgCl}_{2}, 0.5$ theophylline, 5 HEPES, pH 7.5/NaOH). Full-length constructs usually expressed very well on the following day, whereas the functional expression of split channels required 2-3 days. 


\section{Electrophysiological recordings}

2-5 days after RNA injection, membrane currents were recorded at room temperature under two-electrode voltage-clamp, using a TurboTEC 10-CD amplifier (NPI Electronics). Signals were low-pass filtered at $1.3 \mathrm{kHz}$, digitized at $10 \mathrm{kHz}$ with the ITC-16 interface of EPC9 patch-clamp amplifier (HEKA Elektronik), and acquired with PatchMaster software (HEKA Elektronik). The recording pipettes had resistances of 0.3-1 $\mathrm{M} \Omega$, when filled with $2 \mathrm{M} \mathrm{KCl}$. The extracellular medium contained (in mM): $115 \mathrm{NaCl}, 2.5 \mathrm{KCl}, 1.8 \mathrm{CaCl}_{2}, 10 \mathrm{HEPES}$, $\mathrm{pH} 7.2 / \mathrm{NaOH}$. In some experiments, $5 \mathrm{mM} \mathrm{MgCl}_{2}$ was added to the extracellular solution. For measurement of tail currents or constitutively active channels, $\mathrm{K}^{+}$ concentration was elevated to $60 \mathrm{mM}$, replacing $\mathrm{Na}^{+}$. Capacitance currents were compensated with the analog circuitry of the amplifier to the best extent possible. In the case of channels which had no constitutive activity, $\mathrm{P} / 4$ or $\mathrm{P} / 8$ protocol was used to subtract leak and capacitance currents from a holding potential of -100 $\mathrm{mV}$ or $-120 \mathrm{mV}$.

\section{Voltage-clamp fluorometry}

To reduce unspecific labelling of endogenous cysteines on the oocyte surface, we took advantage of the fact that the trafficking mechanisms of Kv10.1 are temperature-dependent. The oocytes were kept at $12{ }^{\circ} \mathrm{C}$ for $3-4$ days after RNA injection, during which time the channel protein was made, but was not sent to the plasma membrane. Subsequently, the oocytes were incubated for $30 \mathrm{~min}$ at room temperature with 0.1-0-5 $\mathrm{mM}$ glycine malemide to block endogenous cysteines, washed extensively with ND-96 and left overnight at room temperature to allow the nascent channels to come to the plasma membrane. On the next day, the oocytes were labelled with $12.5 \mu \mathrm{M}$ TMRM (tetramethylrhodamine malemide, Invitrogen) diluted in depolarizing solution (in mM: $92 \mathrm{KCl}, 1.8 \mathrm{CaCl}_{2}, 10 \mathrm{HEPES}$, $\mathrm{pH} 7.5 / \mathrm{NaOH}$ ) for $1 \mathrm{~h}$ on ice in darkness. After labelling, the oocytes were washed extensively with ND-96 and kept at $16^{\circ} \mathrm{C}$ in darkness until recording. 
VCF signals were recorded through 20x 0.75-NA fluorescence objective (Nikon). The illumination source was a $150 \mathrm{~W}$ xenon lamp. In the light path, HQ535/50 excitation filter, HQ610/75 emission filter and Q565LP dichroic mirror were used (Chroma Technology). The emitted light was collected with a Hamamatsu HC120-05 photomultiplier tube. During fluorescence recordings the oocytes were voltage-clamped, using a Dagan CA1-B amplifier. Fluorescence and current signals were low-pass filtered at $1 \mathrm{kHz}$, digitized at $5 \mathrm{kHz}$ with a Digidata1440A analog-to-digital converter (Axon Instruments), and acquired with pClamp10 software (Axon Instruments). To increase signal to noise ratio, each VCF trace was averaged 10 times.

\section{Single-molecule colocalzation}

Single-molecule imaging was performed 1 day after RNA injection, using Total Internal Reflection Fluorescence Microscopy (TIRFM). Manually devitellinized oocytes were placed on high refractive index coverglass $(n=1.78)$ and imaged through Olympus 100x 1.65-NA oil immersion objective. EGFP-tagged VSDs and full-length subunits were excited with a 488-nm phoxX laser, and mCherry-tagged PDs and full-length subunits were excited with a 593-nm DPSS laser. z488/594rpc polychroic (Chroma) was used as the excitation filter; 525/50 and 629/53 emission filters were used for EGFP and mCherry, respectively. The illuminating beam was set at the critical angle for total internal reflection, so that excitation was limited to the contact area between the specimen and the glass. This allowed us to image channels in the plasma membrane, without acquiring signal from the bulk of the oocyte. mCherry and EGFP were excited sequentially. Movies of 800 frames (200 for mCherry and 600 for EGFP) were captured at the rate of $10 \mathrm{~Hz}$ with iXon DU-897 EMCCD camera (Andor). During acquisition, the fluorescent spots bleached completely.

The bleaching movies of the pore domain tagged with EGFP were acquired in a similar way, using Nikon 100x 1.49 NA oil immersion TIRF objective. Devitellinized oocytes were placed on $35 \mathrm{~mm} \mu$-dishes with glass bottom $(\mathrm{n}=1.52$; IBIDI). They were excited with a 488-nm laser and the light path contained 
525/50-nm emission filter. The movies were captured with iXon DU-897 EMCCD camera (Andor) at $10 \mathrm{~Hz}$.

\section{Data analysis and statistics}

In each experiment, we used oocytes isolated from at least two different frogs. The sample numbers are indicated in the figure legends. For statistical analysis, we used Student's t-test in case means of two groups were compared or analysis of variance followed by multiple comparisons with Bonferroni's correction, if more groups were compared.

Electrophysiological recordings were analysed with IGOR Pro (Wavemetrics) or FitMaster (HEKA Elektronik). The current-voltage curves were constructed by normalizing the current to the maximal value for each oocyte, averaging them and connecting the points representing the mean with straight lines. Conductance and current deactivation kinetics were obtained from biexponential fits to tail currents:

$$
I(t)=A_{1} e^{-\frac{t}{\tau_{1}}}+A_{2} e^{-\frac{t}{\tau_{2}}}+I_{0},
$$

where $I$ is the current, $t$ is the time, $\tau_{1}$ and $\tau_{2}$ are the time constants of the fast and the slow component, A1 and A2 are the corresponding amplitudes, and $\mathrm{I}_{0}$ is the offset current. The conductance is proportional to $I(t=0)$. However, in the case of constitutively active split channels, tail currents were contaminated by a capacitance transient, because $\mathrm{P} / \mathrm{n}$ subtraction was impossible. The conductance was then calculated as the ratio of the steady-state current and the driving force:

$$
G=\frac{I}{V-V_{K}},
$$

where $G$ is the conductance, $I$ is the steady-state current, $V$ is the voltage and $V_{K}$ is the equilibrium potential for $\mathrm{K}^{+}$. Since the constitutively active channels showed inward currents, $V_{K}$ was estimated from the zero-crossing of the current-voltage curve for each oocyte, assuming that $\mathrm{K}^{+}$conductance was dominating. To compare the voltage-dependency between different channels, conductance was normalized to the maximal value, averaged between oocytes expressing the same construct, and fitted with the following Boltzmann function: 


$$
G / G_{\max }=\frac{1}{1+e^{-k\left(V-V_{\text {half }}\right)}},
$$

where $G / G_{\max }$ is the normalized conductance, $k$ is the slope factor $(\mathrm{RT} / \mathrm{zF}), V$ is the voltage, and $V_{h a l f}$ is the voltage for which the conductance is half maximal. Maximum likelihood estimates of $k$ and $V$ half were considered different between groups if the intervals encompassing the mean \pm 3 SD were non-overlapping. Current activation kinetics were compared by quantifying the time needed for current to reach $90 \%$ of its maximal value.

VCF recordings were analysed with Clampfit (Axon Instruments) and IGOR Pro (Wavemetrics). To measure kinetics, activation and deactivation phases of the fluorescence traces were fitted with single exponential functions. Steady-state, voltage-dependent changes of fluorescence were normalized to the maximal value, averaged between oocytes expressing the same channel and fitted with a Boltzmann function equivalent to the one used for conductance.

Colocalization between EGFP and mCherry puncta was analysed manually. The first 3-5 frames of the bleaching movies were averaged for each channel and the relative localization of EGFP and mCherry spots was determined (two spots were considered colocalized if they were within 3 pixels from one another, which corresponds to a distance of $150 \mathrm{~nm}$ ). Only single, stable spots were included in the analysis. Colocalization coefficients presented in the Results section are defined as the number of overlapping spots divided by the sum of overlapping and non-overlapping spots. We used the following equation to subtract densitydependent, random colocalization (the probability of finding two molecules in a diffraction-limited spot just by chance, without interaction between them):

$$
f=\frac{a \times d g \times d r}{d g+d r},
$$

where $f$ is the random colocalization, $a$ is the area of a diffraction-limited spot with a radius of $150 \mathrm{~nm}, d g$ is the density of green puncta and $d r$ is the density of red puncta. 


\section{References}

1. Hille, B., Ion Channels of Excitable Membranes. 2001, Sunderland, MA: Sinauer Associates.

2. Hodgkin, A.L. and A.F. Huxley, A quantitative description of membrane current and its application to conduction and excitation in nerve. J Physiol, 1952. 117(4): p. 500-44.

3. Gutman, G.A., et al., International Union of Pharmacology. LIII. Nomenclature and molecular relationships of voltage-gated potassium channels. Pharmacol Rev, 2005. 57(4): p. 473-508.

4. Urrego, D., et al., Potassium channels in cell cycle and cell proliferation. Philosophical Transactions of the Royal Society B: Biological Sciences, 2014. 369(1638).

5. Wulff, H., N.A. Castle, and L.A. Pardo, Voltage-gated potassium channels as therapeutic targets. Nat Rev Drug Discov, 2009. 8(12): p. 982-1001.

6. Lu, Z., A.M. Klem, and Y. Ramu, lon conduction pore is conserved among potassium channels. Nature, 2001. 413(6858): p. 809-13.

7. Stühmer, W., et al., Structural parts involved in activation and inactivation of the sodium channel. Nature, 1989. 339(6226): p. 597-603.

8. Papazian, D.M., et al., Alteration of voltage-dependence of Shaker potassium channel by mutations in the S4 sequence. Nature, 1991. 349(6307): p. 305-10.

9. Armstrong, C.M. and F. Bezanilla, Currents related to movement of the gating particles of the sodium channels. Nature, 1973. 242(5398): p. 459-61.

10. Jiang, Y., et al., X-ray structure of a voltage-dependent $K+$ channel. Nature, 2003. 423(6935): p. 33-41.

11. Long, S.B., E.B. Campbell, and R. Mackinnon, Crystal structure of a mammalian voltage-dependent Shaker family K+ channel. Science, 2005. 309(5736): p. 897-903.

12. Long, S.B., et al., Atomic structure of a voltage-dependent $\mathrm{K}+$ channel in a lipid membrane-like environment. Nature, 2007. 450(7168): p. 376-82.

13. Ramu, Y., Y. Xu, and Z. Lu, Enzymatic activation of voltage-gated potassium channels. Nature, 2006. 442(7103): p. 696-9.

14. Xu, Y., Y. Ramu, and Z. Lu, Removal of phospho-head groups of membrane lipids immobilizes voltage sensors of $K+$ channels. Nature, 2008. 451(7180): p. 826-9.

15. $\mathrm{Xu}, \mathrm{Y} ., \mathrm{Y} . \mathrm{Ramu}$, and $\mathrm{Z}$. $\mathrm{Lu}, \mathrm{A}$ shaker $\mathrm{K}+$ channel with a miniature engineered voltage sensor. Cell, 2010. 142(4): p. 580-9.

16. Adaixo, R., et al., Structural properties of PAS domains from the KCNH potassium channels. PLoS One, 2013. 8(3): p. e59265.

17. Marques-Carvalho, M.J., et al., Structural, biochemical, and functional characterization of the cyclic nucleotide binding homology domain from the mouse EAG1 potassium channel. J Mol Biol, 2012. 423(1): p. 34-46.

18. Haitin, Y., A.E. Carlson, and W.N. Zagotta, The structural mechanism of KCNH-channel regulation by the eag domain. Nature, 2013. 501(7467): p. 444-8.

19. Schoppa, N.E., et al., The size of gating charge in wild-type and mutant Shaker potassium channels. Science, 1992. 255(5052): p. 1712-5.

20. Seoh, S.A., et al., Voltage-sensing residues in the S2 and S4 segments of the Shaker K+ channel. Neuron, 1996. 16(6): p. 1159-67.

21. Aggarwal, S.K. and R. MacKinnon, Contribution of the S4 segment to gating charge in the Shaker K+ channel. Neuron, 1996. 16(6): p. 1169-77.

22. Jiang, Y., et al., The principle of gating charge movement in a voltage-dependent $K+$ channel. Nature, 2003. 423(6935): p. 42-8. 
23. Ruta, V., J. Chen, and R. MacKinnon, Calibrated measurement of gating-charge arginine displacement in the KvAP voltage-dependent $K+$ channel. Cell, 2005. 123(3): p. 463-75.

24. Gandhi, C.S., et al., The orientation and molecular movement of a $k(+)$ channel voltagesensing domain. Neuron, 2003. 40(3): p. 515-25.

25. Chanda, B., et al., Gating charge displacement in voltage-gated ion channels involves limited transmembrane movement. Nature, 2005. 436(7052): p. 852-6.

26. Posson, D.J., et al., Small vertical movement of a $K+$ channel voltage sensor measured with luminescence energy transfer. Nature, 2005. 436(7052): p. 848-51.

27. Starace, D.M. and F. Bezanilla, A proton pore in a potassium channel voltage sensor reveals a focused electric field. Nature, 2004. 427(6974): p. 548-53.

28. Tombola, F., et al., The twisted ion-permeation pathway of a resting voltage-sensing domain. Nature, 2007. 445(7127): p. 546-9.

29. Ahern, C.A. and R. Horn, Focused electric field across the voltage sensor of potassium channels. Neuron, 2005. 48(1): p. 25-9.

30. Tombola, F., M.M. Pathak, and E.Y. Isacoff, How far will you go to sense voltage? Neuron, 2005. 48(5): p. 719-25.

31. Vargas, E., et al., An emerging consensus on voltage-dependent gating from computational modeling and molecular dynamics simulations. J Gen Physiol, 2012. 140(6): p. 587-94.

32. Slesinger, P.A., Y.N. Jan, and L.Y. Jan, The S4-S5 loop contributes to the ion-selective pore of potassium channels. Neuron, 1993. 11(4): p. 739-49.

33. Sanguinetti, M.C. and Q.P. Xu, Mutations of the S4-S5 linker alter activation properties of HERG potassium channels expressed in Xenopus oocytes. J Physiol, 1999. 514 ( Pt 3): p. 667-75.

34. Van Slyke, A.C., et al., Mutations within the S4-S5 linker alter voltage sensor constraints in hERG K+ channels. Biophys J, 2010. 99(9): p. 2841-52.

35. Haddad, G.A. and R. Blunck, Mode shift of the voltage sensors in Shaker K+ channels is caused by energetic coupling to the pore domain. J Gen Physiol, 2011. 137(5): p. 455-72.

36. Labro, A.J., et al., The S4-S5 linker of KCNQ1 channels forms a structural scaffold with the $S 6$ segment controlling gate closure. J Biol Chem, 2011. 286(1): p. 717-25.

37. Hull, C.M., et al., Regional flexibility in the S4-S5 linker regulates $h E R G$ channel closedstate stabilization. Pflugers Arch, 2014. 466(10): p. 1911-9.

38. Lu, Z., A.M. Klem, and Y. Ramu, Coupling between voltage sensors and activation gate in voltage-gated K+ channels. J Gen Physiol, 2002. 120(5): p. 663-76.

39. Labro, A.J., et al., Kv channel gating requires a compatible S4-S5 linker and bottom part of S6, constrained by non-interacting residues. J Gen Physiol, 2008. 132(6): p. 66780.

40. Tristani-Firouzi, M., J. Chen, and M.C. Sanguinetti, Interactions between S4-S5 linker and 66 transmembrane domain modulate gating of HERG K+ channels. J Biol Chem, 2002. 277(21): p. 18994-9000.

41. Ferrer, T., et al., The S4-S5 linker directly couples voltage sensor movement to the activation gate in the human ether-a'-go-go-related gene (hERG) K+ channel. J Biol Chem, 2006. 281(18): p. 12858-64.

42. Long, S.B., E.B. Campbell, and R. Mackinnon, Voltage sensor of Kv1.2: structural basis of electromechanical coupling. Science, 2005. 309(5736): p. 903-8.

43. Jensen, M.O., et al., Mechanism of voltage gating in potassium channels. Science, 2012. 336(6078): p. 229-33.

44. Jensen, M.O., et al., Principles of conduction and hydrophobic gating in $K+$ channels. Proc Natl Acad Sci U S A, 2010. 107(13): p. 5833-8. 
45. Ledwell, J.L. and R.W. Aldrich, Mutations in the S4 region isolate the final voltagedependent cooperative step in potassium channel activation. J Gen Physiol, 1999. 113(3): p. 389-414.

46. Islas, L.D. and F.J. Sigworth, Voltage sensitivity and gating charge in Shaker and Shab family potassium channels. J Gen Physiol, 1999. 114(5): p. 723-42.

47. Pathak, M., et al., The cooperative voltage sensor motion that gates a potassium channel. J Gen Physiol, 2005. 125(1): p. 57-69.

48. Osteen, J.D., et al., KCNE1 alters the voltage sensor movements necessary to open the KCNQ1 channel gate. Proc Natl Acad Sci U S A, 2010. 107(52): p. 22710-5.

49. Osteen, J.D., et al., Allosteric gating mechanism underlies the flexible gating of KCNQ1 potassium channels. Proc Natl Acad Sci U S A, 2012. 109(18): p. 7103-8.

50. Terlau, H., et al., Extracellular Mg2+ regulates activation of rat eag potassium channel. Pflugers Arch, 1996. 432(2): p. 301-12.

51. Schonherr, R., et al., Conformational switch between slow and fast gating modes: allosteric regulation of voltage sensor mobility in the EAG K+ channel. Neuron, 2002. 35(5): p. 935-49.

52. Hines, K.E., T.R. Middendorf, and R.W. Aldrich, Determination of parameter identifiability in nonlinear biophysical models: A Bayesian approach. J Gen Physiol, 2014. 143(3): p. 401-16.

53. Chowdhury, S. and B. Chanda, Estimating the voltage-dependent free energy change of ion channels using the median voltage for activation. J Gen Physiol, 2012. 139(1): p. 3-17.

54. Wang, S., et al., Structural dynamics of potassium-channel gating revealed by singlemolecule FRET. Nat Struct Mol Biol, 2016. 23(1): p. 31-6.

55. Chowdhury, S. and B. Chanda, Perspectives on: conformational coupling in ion channels: thermodynamics of electromechanical coupling in voltage-gated ion channels. J Gen Physiol, 2012. 140(6): p. 613-23.

56. Santos, J.S., S.M. Grigoriev, and M. Montal, Molecular template for a voltage sensor in a novel $K+$ channel. III. Functional reconstitution of a sensorless pore module from a prokaryotic Kv channel. J Gen Physiol, 2008. 132(6): p. 651-66.

57. Santos, J.S., et al., Crystal structure of a voltage-gated $K+$ channel pore module in a closed state in lipid membranes. J Biol Chem, 2012. 287(51): p. 43063-70.

58. Syeda, R., et al., Tetrameric assembly of $K v L m K+$ channels with defined numbers of voltage sensors. Proc Natl Acad Sci U S A, 2012. 109(42): p. 16917-22.

59. McCusker, E.C., et al., Simplified bacterial "pore" channel provides insight into the assembly, stability, and structure of sodium channels. J Biol Chem, 2011. 286(18): p. 16386-91.

60. Shaya, D., et al., Voltage-gated sodium channel (NaV) protein dissection creates a set of functional pore-only proteins. Proc Natl Acad Sci U S A, 2011. 108(30): p. 12313-8.

61. McCusker, E.C., et al., Structure of a bacterial voltage-gated sodium channel pore reveals mechanisms of opening and closing. Nat Commun, 2012. 3: p. 1102.

62. Tao, X., et al., A gating charge transfer center in voltage sensors. Science, 2010. 328(5974): p. 67-73.

63. Lacroix, J.J., et al., Moving gating charges through the gating pore in a Kv channel voltage sensor. Proc Natl Acad Sci U S A, 2014. 111(19): p. E1950-9.

64. Faure, E., et al., A limited 4 A radial displacement of the S4-S5 linker is sufficient for internal gate closing in Kv channels. J Biol Chem, 2012. 287(47): p. 40091-8.

65. Diana Urrego, N.M., Roser Ufartes, and Luis A. Pardo, Periodic expression of Kv10.1 driven by $p R b / E 2 F 1$ contributes to G2/M progression of cancer and non-transformed cells. CELL CYCLE, 2016: p. 13. 
66. Hemmerlein, B., et al., Overexpression of Eag1 potassium channels in clinical tumours. Mol Cancer, 2006. 5: p. 41.

67. Kortum, F., et al., Mutations in KCNH1 and ATP6V1B2 cause Zimmermann-Laband syndrome. Nat Genet, 2015. 47(6): p. 661-7.

68. Simons, C., et al., Mutations in the voltage-gated potassium channel gene KCNH1 cause Temple-Baraitser syndrome and epilepsy. Nat Genet, 2015. 47(1): p. 73-7.

69. Mortensen, L.S., et al., KV10.1 opposes activity-dependent increase in Ca2+ influx into the presynaptic terminal of the parallel fibre - Purkinje cell synapse. J Physiol, 2014.

70. Lorinczi, E., et al., Voltage-dependent gating of KCNH potassium channels lacking a covalent link between voltage-sensing and pore domains. Nat Commun, 2015. 6: p. 6672.

71. Ulbrich, M.H. and E.Y. Isacoff, Subunit counting in membrane-bound proteins. Nat Methods, 2007. 4(4): p. 319-21.

72. Chen, Y., et al., Subunit composition of a DEG/ENaC mechanosensory channel of Caenorhabditis elegans. Proc Natl Acad Sci U S A, 2015. 112(37): p. 11690-5.

73. Thouta, S., et al., Proline scan of the HERG channel $S 6$ helix reveals the location of the intracellular pore gate. Biophys J, 2014. 106(5): p. 1057-69.

74. de la Pena, P., et al., Demonstration of physical proximity between the $N$ terminus and the S4-S5 linker of the human ether-a-go-go-related gene ( $h E R G$ ) potassium channel. J Biol Chem, 2011. 286(21): p. 19065-75.

75. de la Pena, P., et al., Interactions between the $\mathrm{N}$-terminal tail and the gating machinery of $h E R G K(+)$ channels both in closed and open/inactive states. Pflugers Arch, 2015. 467(8): p. 1747-56. 
Appendices 


\section{Curriculum Vitae}

\section{Education}

09.2004-05.2007 International Baccalaureate Bilingual Diploma awarded International Baccalaureate School No.1002, Poznan, Poland

10.2007-06.2011 B.Sc. in Neuroscience awarded with a distinction Completed two years of course in Psychology Jagiellonian University, Krakow, Poland

09.2011-present International M.Sc./PhD Program Georg-August-Universität, Göttingen

International Max Planck Research School Neurosciences

09.2012-present $\quad$ PhD project

Max Planck Institute for Experimental Medicine, Göttingen Department Molecular Biology of Neuronal Signals Oncophysiology Group

Supervisors: Prof. Luis A. Pardo, Prof. Walter Stühmer

\section{Scholarships:}

10.2008-06.2011 Scholarship for Academic Results, Jagiellonian University

09.2011-08.2012 Scholarship for the Promotion of the Max Planck Society

\section{Publications:}

Tomczak AP, Fernandez-Trillo J, Bharril S, Papp F, Panyi G, Stühmer W, Isacoff EY, Pardo LA. Interactions between voltage-sensor and pore domains revealed by split Kv10.1 channels. In prep.

Lörinczi E., Gomez-Posada JC, de la Pena P, Tomczak AP, Fernandez-Trillo J, Leipscher U, Stühmer W, Barros F, Pardo LA. Voltage-dependent gating of KCNH potassium channels lacking a covalent link between voltage-sensing and pore domains. Nat Commun, 2015. 6: p. 6672.

Urrego D, Tomczak AP, Zahed F, Stühmer W, Pardo LA. Potassium channels in cell cycle and cell proliferation. Philosophical Transactions of the Royal Society B: Biological Sciences, 2014. 369 (1638). 


\section{PHILOSOPHICAL TRANSACTIONS}

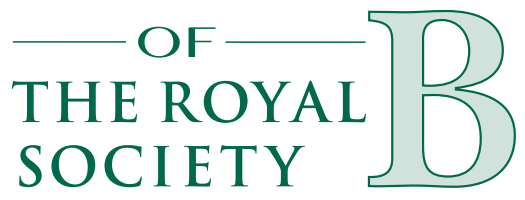

\section{rstb.royalsocietypublishing.org}

\section{Review}
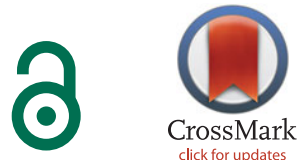

Cite this article: Urrego D, Tomczak AP,

Zahed F, Stühmer W, Pardo LA. 2014

Potassium channels in cell cycle

and cell proliferation. Phil. Trans. R. Soc. B 369:

20130094.

http://dx.doi.org/10.1098/rstb.2013.0094

One contribution of 17 to a Theme Issue 'Ion channels, transporters and cancer'.

\author{
Subject Areas: \\ physiology, cellular biology, biophysics, \\ neuroscience
}

\section{Keywords:}

potassium channels, proliferation, cell cycle, cell division, cancer

\section{Author for correspondence:}

Luis A. Pardo

e-mail: pardo@em.mpg.de

\section{Potassium channels in cell cycle and cell proliferation}

Diana Urrego ${ }^{1}$, Adam P. Tomczak ${ }^{1}$, Farrah Zahed ${ }^{1}$, Walter Stühmer ${ }^{2,3}$

and Luis A. Pardo ${ }^{1}$

${ }^{1}$ Oncophysiology Group, and ${ }^{2}$ Department of Molecular Biology of Neuronal Signals, Max Planck Institute of Experimental Medicine, Hermann-Rein-Strasse 3, Göttingen 37075, Germany

${ }^{3}$ Cluster of Excellence Nanoscale Microscopy and Molecular Physiology of the Brain, Göttingen, Germany

Normal cell-cycle progression is a crucial task for every multicellular organism, as it determines body size and shape, tissue renewal and senescence, and is also crucial for reproduction. On the other hand, dysregulation of the cell-cycle progression leading to uncontrolled cell proliferation is the hallmark of cancer. Therefore, it is not surprising that it is a tightly regulated process, with multifaceted and very complex control mechanisms. It is now well established that one of those mechanisms relies on ion channels, and in many cases specifically on potassium channels. Here, we summarize the possible mechanisms underlying the importance of potassium channels in cell-cycle control and briefly review some of the identified channels that illustrate the multiple ways in which this group of proteins can influence cell proliferation and modulate cell-cycle progression.

\section{Introduction}

Regulation of cell division is of great relevance for eukaryotes. Cells must proliferate throughout ontogenesis, tissue renewal and remodelling, and to repair damaged areas during wound healing. Defective cell-cycle checkpoints are a common feature of cancer cells and the inactivation of cell cycle regulators decides the physiological or pathological fate of stem cells. Although there are a large number of studies on the molecular and biochemical mechanisms controlling the cell cycle, the bioelectrical modulation of cell-cycle progression is still poorly understood. $\mathrm{K}^{+}$channels have been implicated in the control of cellcycle progression both through their influence on the membrane potential and non-canonical, permeation-independent mechanisms.

\section{Checkpoints and transmembrane potential regulate cell-cycle progression}

The process that produces two daughter cells from a mother cell has been divided into several phases, each with very characteristic functional properties. Cell division in eukaryotes starts with the G1 (gap 1) phase, which separates the previous cell division from the period of DNA synthesis (S-phase), where chromosome replication is accomplished. This is followed by the second gap (G2) and the mitotic (M) phase. After M phase, a cell can proceed to a new G1 phase or enter a quiescent state (termed G0) that can last for a very long time, even for the rest of the life of the cell in the case of end-differentiated cells. The correct progression of the cycle is guaranteed because the initiation of a late event is strictly dependent on the successful completion of the preceding step. In eukaryotic cells, for example, mitosis will not start until the completion of DNA synthesis. The interdependency of events is owing to a series of surveillance or control mechanisms termed checkpoints, which have evolved to minimize the production and propagation of genetic inaccuracies [1,2]. The complex machinery of cell-cycle checkpoints includes in all cases a sensor supervising the completeness of a particular task and a response element triggering the next downstream 


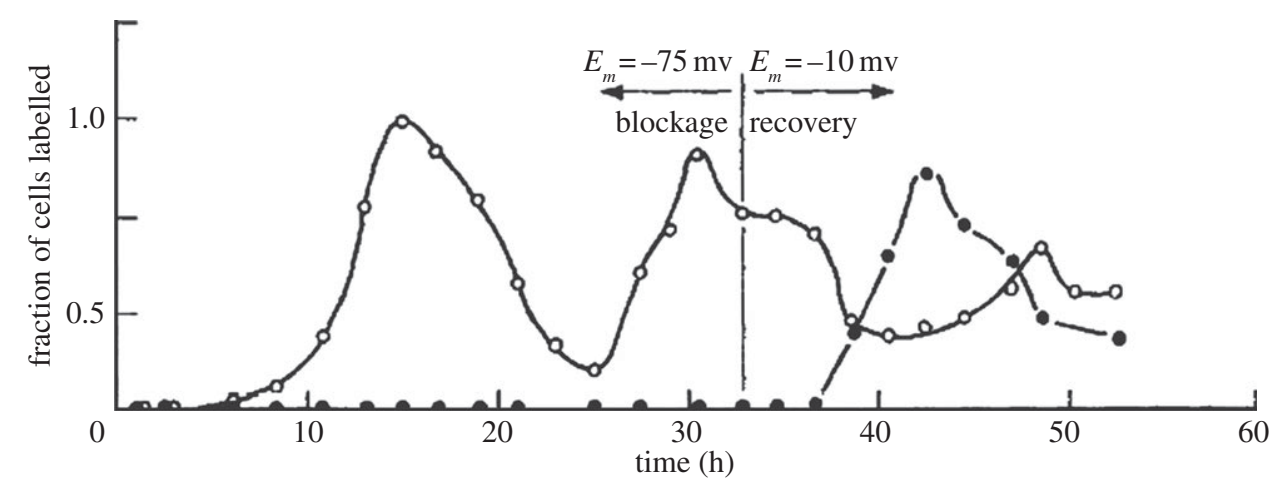

Figure 1. Complete block of DNA synthesis, measures as $\left[{ }^{3} \mathrm{H}\right]$ thymidine incorporation in cells with fixed hyperpolarized membrane potential. Reproduced from [7] with permission. Open circles, control; black circles, manipulation of membrane potential.

event, which will be a process involved in the actual replication and segregation of the DNA. For instance, the downstream event at the onset of $S$ phase is DNA synthesis, the downstream event at the onset of mitosis is the assembly of the spindle and the downstream event at the end of mitosis is chromosome segregation $[3,4]$. Thus, checkpoints are constitutive feedback control pathways safeguarding key cell-cycle transitions G1/S, G2/M and exit from mitosis [5]. The key components of the mechanisms coordinating the downstream events are cyclin/cyclin-dependent kinase (CDK) complexes, which need to be expressed in a timely fashion and/or activated to allow cell-cycle progression.

The transmembrane potential has been reported as a cellular bioelectric parameter that influences the progression through the cell cycle [6]. The concept came from the early experimental observation of a correlation between the resting membrane potential and the degree of mitotic activity [7]; forcing the membrane potential of Chinese hamster ovary cells to a fixed hyperpolarized value completely inhibited DNA synthesis measured as $\left[{ }^{3} \mathrm{H}\right]$ thymidine incorporation, while cycling was recovered upon release of the potential (figure 1). Cell types with a very hyperpolarized resting potential, such as muscle cells and neurons, typically show little or no mitotic activity. Inversely, it was reported in the early 1970s that ouabain-induced depolarization was followed by the initiation of DNA synthesis and subsequent mitosis in chick spinal cord neurons [8,9]. Moreover, it has been shown that the membrane potential is not constant during progression through the cell cycle $[10,11]$. For example, the distribution of membrane potentials in cells from the breast cancer cell line MCF-7 is multimodal. The frequency of events at each maximum can be shifted when experimental treatments change the distribution of cells among the different phases of the cell cycle. The results of these experiments showed a pattern of positive correlation where the membrane potential hyperpolarizes during the G1/S transition, there is a significant contribution of depolarized cells towards G0/G1 and an enrichment in hyperpolarized cells towards G2/M transition [12].

\section{3. $\mathrm{K}^{+}$channels as important players in the cell cycle}

If the membrane potential is not constant along the cell cycle, cell-cycle-dependent changes in membrane permeability are required (figure 2). Potassium conductance governs the resting membrane potential in both excitable and non-excitable cells.
In contrast to an action potential fired by a neuron, the potential changes along the cell cycle are much slower, gradual and smaller, and can be intuitively explained by modifications in the conductance that sets the resting membrane potential. Proliferation was one of the first identified aspects of cell physiology where potassium channels play a crucial role. The early observation that wide-spectrum potassium channel blockers inhibit proliferation [13] has been repeatedly confirmed in many tissues and cell types (reviewed e.g. in [6]). Many different potassium channels show cell-cycle-dependent variations of expression or activity [14-17].

For instance, a large conductance, voltage-gated $\mathrm{K}^{+}$channel is expressed in unfertilized mouse oocytes; in the first cell cycle of fertilized oocytes, the channel is active throughout $M$ and G1 phases, and inactive during S and G2. Thus, changes in channel activity set the membrane potential along the cell cycle in the oocyte [18]. Increasing evidence shows that voltage-gated potassium channels are required for proliferation and may also help to determine the final identity and morphology of the cell [19-22]. The results of experiments in lymphocytes where the inhibition of $\mathrm{K}^{+}$channel activity induces a reversible cell-cycle arrest $[23,24]$ or experiments where potassium channel blockers inhibit Schwann cell proliferation in a dose-dependent manner $[22,25,26]$ have been replicated in many systems and by many approaches; data from those experiments have been compiled already in several reviews (e.g. [27-31]).

Direct evidence for a change in ion channel composition in G1 phase was obtained from embryonic retinal cells, which express mainly two membrane conductances, delayed rectifier $\left(\mathrm{I}_{\mathrm{K}}\right)$ and inward rectifier $\left(\mathrm{I}_{\mathrm{Kir}}\right)$ potassium currents [32]. Daughters of the same parental cell examined during and after mitosis always expressed similar $\mathrm{I}_{\mathrm{K}}$ and $\mathrm{I}_{\mathrm{Kir}}$ densities. However, non-sibling cells showed quantitative and qualitative differences in $\mathrm{I}_{\mathrm{K}}$ and $\mathrm{I}_{\mathrm{Kir}}$ densities. The heterogeneity therefore arises after cells re-enter G1, because the density distribution of potassium channels at cytokinesis is shown to be symmetric in both daughter cells [33].

The mechanisms controlling ion channel densities along the cell cycle appear to be manifold. For example, $\mathrm{K}^{+}$channel activity in mouse oocytes is at least partly independent of the nuclear cell-cycle clock, because channel activity continues to cycle in bisected embryos in the anucleate as well as the nucleate fragments [34]. This suggests the active contribution of the cytoplasmic cell-cycle clock, which may involve changes induced by surface contractions and deformations before the cleavage of daughter cells on the channel activity $[34,35]$. 


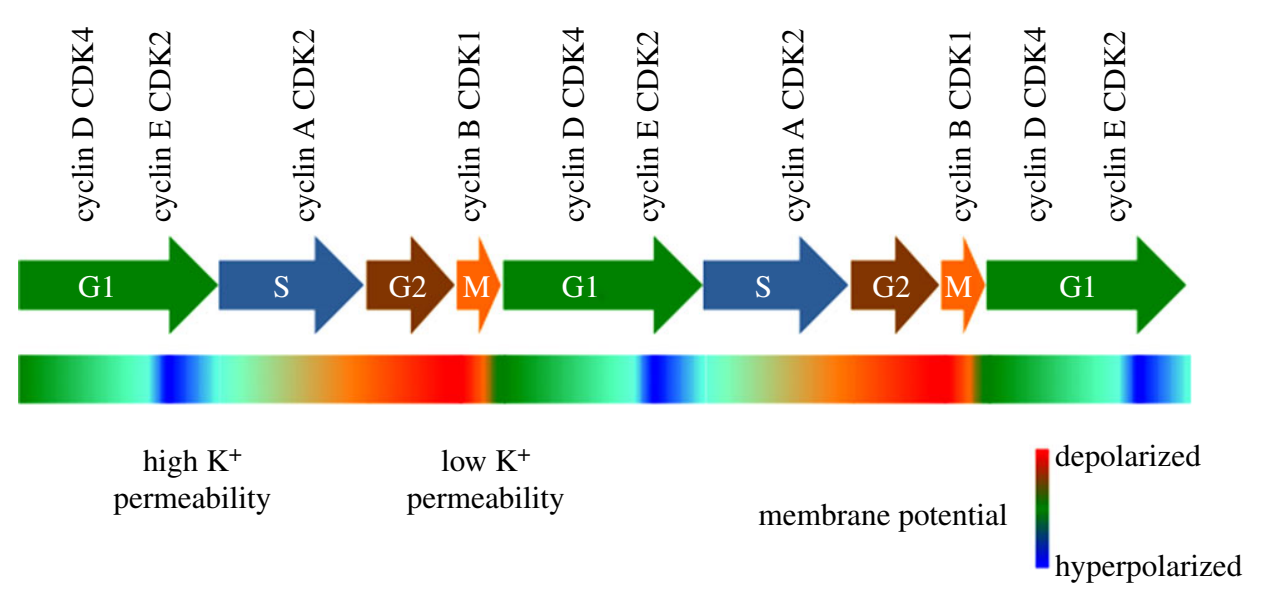

Figure 2. Schematic of the behaviour of the membrane potential along the cell cycle. Different potassium channels show variations of expression or activity through the cell cycle, thus shifting the membrane potential towards hyperpolarized values, close to the equilibrium potential for potassium, at the border between $\mathrm{G} 1$ and S-phases.

Thus, potassium channels are proposed to be involved in the signal transduction elicited by cell-cycle checkpoints, and help to elicit cell responses in the cell-cycle machinery, integrating the nuclear clock and the cytoplasmic cell-cycle clock. Pointing towards this hypothesis, there have been reports where $\mathrm{K}^{+}$channel blockers (TEA) and depolarizing agents (veratridine) inhibit cell proliferation in oligodendrocyte progenitors in cell culture and cerebellar tissue slices, inducing G1 arrest through accumulation of $\mathrm{p} 27^{\mathrm{kip} 1}$ and $\mathrm{p} 21^{\mathrm{CIP} 1}$, two CDK inhibitors known to regulate cell proliferation [36,37].

\section{Importance of $\mathrm{K}^{+}$channels relies on both ionic conduction and permeation- independent mechanisms}

The participation of $\mathrm{K}^{+}$channels in the control of cell cycle could be an early event in evolution. The pore structure and the selectivity filter have been conserved between the prokaryotic and eukaryotic $\mathrm{K}^{+}$channels [38], which suggests that they evolved very early. The importance of $\mathrm{K}^{+}$channels in the cell-cycle progression can also be illustrated in plant cells, for which $\mathrm{K}^{+}$is a major nutrient. BY-2 tobacco cells require an increase in the $\mathrm{K}^{+}$concentration in order to re-enter the cell cycle. The elevated $\mathrm{K}^{+}$concentration increases the turgor pressure, which is required for cell growth. This is achieved by the activity of the inward rectifier $\mathrm{K}^{+}$uptake channels [39]. By contrast, mitosis requires a transient decrease in turgor pressure owing to $\mathrm{K}^{+}$efflux channels. In what could be a reminiscence of this function, the role of $\mathrm{K}^{+}$channels in homoeostatic cell volume regulation is well established, and they play a role in cell volume changes along the cell cycle $[40,41]$. For instance, in a subset of human medulloblastomas, a voltage-gated $\mathrm{K}^{+}$channel $\left(\mathrm{K}_{\mathrm{V}} 10.2\right)$ seems to be required for the completion of mitosis, because it participates in cell volume reduction prior to cytokinesis [21].

$\mathrm{K}^{+}$channels also provide the driving force required for $\mathrm{Ca}^{2+}$ to enter the cell by shifting the membrane potential towards negative values. $\mathrm{Ca}^{2+}$ is an important mediator of intracellular signals implicated in the control of proliferation among other crucial processes in cell physiology, and by keeping the membrane potential at hyperpolarized values, $\mathrm{K}^{+}$ channels ensure efficient $\mathrm{Ca}^{2+}$ entry into the cell [42-45].
Still, regardless of whether the potassium gradient is used to generate driving force for $\mathrm{Ca}^{2+}$ or to change the cell volume, we traditionally tend to define the potassium current as the only effector, and ignore possible additional actions of the ion channel molecule itself. If only $\mathrm{K}^{+}$flow was required, essentially any potassium channel expressed at the right moment would be able to affect cell-cycle progression. Experimental observations using either siRNA knockdown or specific blockers, for example antibodies, have repeatedly shown, however, that a specific potassium channel can be important for proliferation (e.g. [46-50]). This would indicate a permeation-independent, non-canonical mechanism that could involve protein-protein interactions, dependent or independent of the conformational changes of the channel mediated by voltage. Non-canonical functions [51] have been described for at least the Drosophila eag channel [52], its mammalian orthologue $\mathrm{K}_{\mathrm{V}} 10.1$ [53], $\mathrm{K}_{\mathrm{V}} 1.3$ [54] and $\mathrm{K}_{\mathrm{Ca}} 3.1$ [55], which are still able influence cell proliferation in the absence of $\mathrm{K}^{+}$permeation. Moreover, an alternatively spliced form of Drosophila eag that lacks the transmembrane regions, and therefore is not even a bona fide potassium channel has also been reported to influence intracellular signalling and alter cell morphology in the background of PKA/PKC activation [56].

In more general terms, the fact that more than 70 genes encode $\mathrm{K}^{+}$channels suggests an exquisite distribution of functions among specific molecular entities, rather than a homogeneous function for all potassium channels. Along these lines, the variability of $\mathrm{K}^{+}$channels is further increased by the formation of heteromultimers, the influence of accessory subunits and a large number of post-translational modifications, such as glycosylation [57], phosphorylation [58] and sumoylation [59]. There is substantial evidence that several $\mathrm{K}^{+}$channels play a role in cell cycle and proliferation by means of both permeation-related and unrelated mechanisms (figure 3). Below, we describe some of them in more detail.

\section{5. $K_{V} 1.3$}

$\mathrm{K}_{\mathrm{V}}$ 1.3 (together with $\mathrm{K}_{\mathrm{Ca}}$ 3.1) was probably the first case showing the involvement of $\mathrm{K}^{+}$channels in cell proliferation $[13,60]$. In a very early report on $\mathrm{T}$ lymphocytes, mitogenesis induced by phytohaemagglutinin caused $\mathrm{K}^{+}$channels to open more rapidly and at more negative membrane 


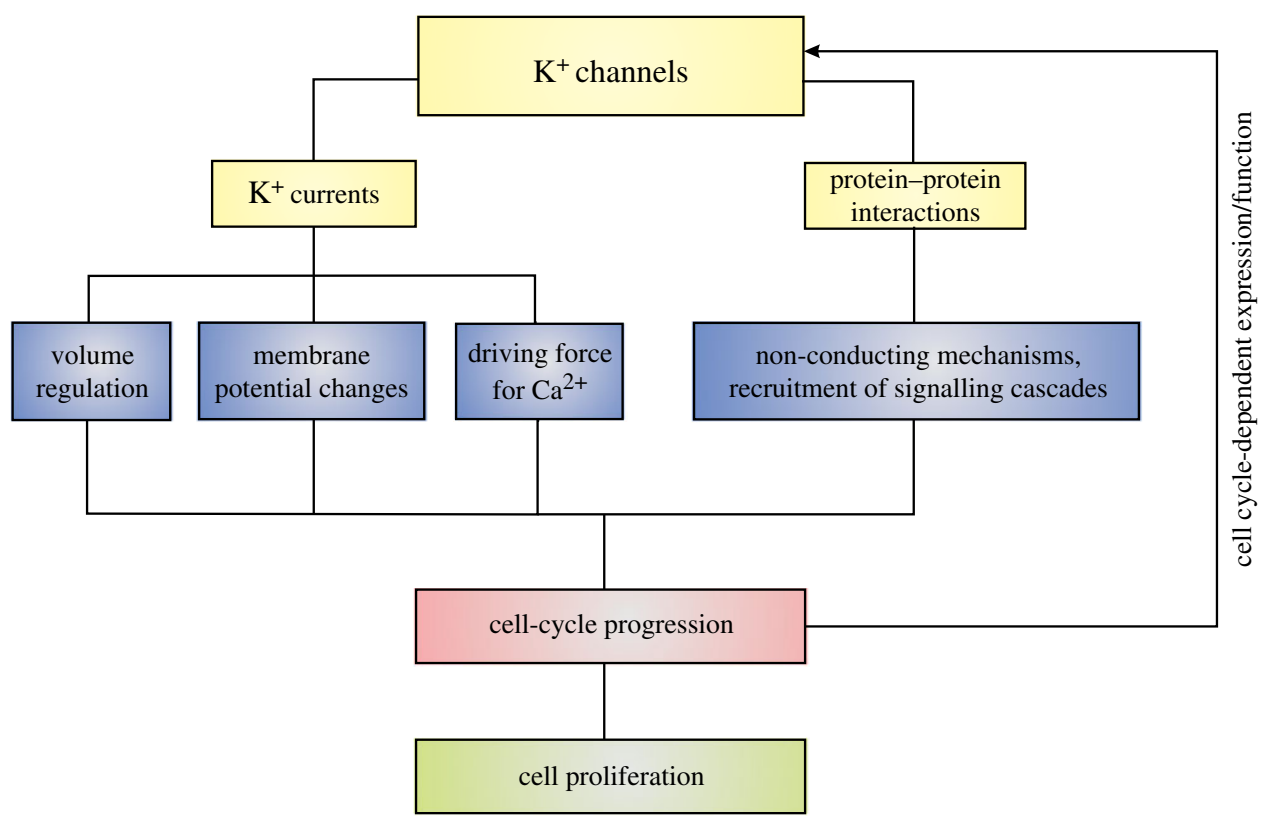

Figure 3. $\mathrm{K}^{+}$channels influence cell-cycle progression through permeation-related and non-canonical mechanisms. The former include cell volume regulation, modulation of membrane potential and generation of driving for $\mathrm{Ca}^{2+}$, while the latter rely on protein-protein interactions. $\mathrm{K}^{+}$channel expression or function can in turn be regulated by progression through the cell cycle.

potentials, suggesting that they may play a role in mitogenesis [13]. $\mathrm{K}_{\mathrm{V}} 1.3$ blockade was shown to suppress T-cell activation and $\mathrm{Ca}^{2+}$ signalling in human $\mathrm{T}$ cells owing to membrane depolarization, resulting in a reduced driving force for $\mathrm{Ca}^{2+}$ entry and impairment of activation by agents inducing mitogenesis [61,62]. $\mathrm{K}_{\mathrm{V}} 1.3$ can act in conjunction with $\mathrm{K}_{\mathrm{Ca}} 3.1$, which is a $\mathrm{Ca}^{2+}$-dependent $\mathrm{K}^{+}$channel activated by $\mathrm{Ca}^{2+}$-calmodulin [63]. $\mathrm{K}_{\mathrm{V}} 1.3$ and $\mathrm{K}_{\mathrm{Ca}} 3.1$ have been found to cluster at the immunological synapse following contact with an antigen-presenting cell [60]. Together, $\mathrm{K}_{\mathrm{V}} 1.3$ and $\mathrm{K}_{\mathrm{Ca}} 3.1$ modulate calcium-dependent cellular processes in immune cells, such as T-cell activation and proliferation $[43,64] . K_{\mathrm{Ca}} 3.1$ has also been implicated in the control of cell proliferation in rat mesenchymal stem cells, vascular smooth muscle cells (VSMCs), hepatocellular carcinoma cells as well as endometrial and prostate cancer cells [45,46,65-68], although in glioma cells $\mathrm{K}_{\mathrm{Ca}} 3.1$ knockdown abolished the current but did not affect proliferation [69]. As $\mathrm{K}_{\mathrm{Ca}} 3.1$ seems to play a crucial role in glioma cell migration [70-75], it might be difficult to dissect both properties and the results can depend very strongly on the methods used to determine proliferation.

$\mathrm{K}_{\mathrm{V}} 1.3$ has also been implicated in the control of the cell cycle in many other cell types, such active microglia cells [76,77], proliferating oligodendrocyte progenitors during G1/S transition [37] and macrophages [78-80]. In human endothelial cells, vascular endothelial growth factor induces a $\mathrm{K}_{\mathrm{V}}$ 1.3-dependent hyperpolarization that results in an increased $\mathrm{Ca}^{2+}$ entry, which is responsible for the effects on proliferation $[81,82]$. It has been shown that the contractile activity of VSMCs controlling blood flow changes during the course of several vascular disorders and the cells acquire a proliferative and migratory phenotype [83]. $\mathrm{K}_{\mathrm{V}} 1.3$ functional expression is associated with the proliferative phenotype, because the blockade of the channel induces a significant inhibition of cell proliferation $[81,84,85]$. Switching from contractile to proliferative phenotype is thus associated with changes in ion channel activity. However, one study suggests
$\mathrm{K}_{\mathrm{V}} 1.3$ increases VSMC proliferation by voltage-dependent conformational changes of the channel that activate intracellular signalling pathways, rather than by ionic conduction [54].

\section{6. $\mathrm{K}_{\mathrm{v}} 11.1$}

The voltage-sensitive human ether à go-go-related gene (hERG, $\mathrm{K}_{\mathrm{V}}$ 11.1) [86] potassium channels have emerged as regulators of both proliferation and survival in cancer cells. $\mathrm{K}_{\mathrm{V}} 11.1$ (encoded by $\mathrm{KCNH} 2$ ) channel expression in normal adult human tissue is abundant in heart, brain, myometrium, pancreas and haematopoietic progenitors [87-90]. $\mathrm{K}_{\mathrm{V}} 11.1$ expression has been reported in many cancer types as well as cancer cell lines of different lineages, be it epithelial, leukemic, connective or neuronal [89-91] Various studies have demonstrated this expression to be largely confined to neoplastic cells both in solid and haematological malignancies, when compared with neighbouring normal tissues or normal bone marrow samples [90-94]. Studies over the past decade have also shown its expression to be preferential to the cancer stem cells especially in leukaemia when compared with normal haematopoietic stem cells [90,94]. $\mathrm{K}_{\mathrm{V}} 11.1$ expression has also been linked to higher grade and worse prognosis, both in the case of solid as well as haematological malignancies [89,91-94]. $\mathrm{K}_{\mathrm{V}} 11.1$ expression is not an epiphenomenon of cancer cells and rather plays a relevant role in their proliferative capacity, for both haematological as well as solid tumours [49,90-98]. Studies by various groups on $\mathrm{K}_{\mathrm{V}} 11.1$ inhibition in cell lines derived from solid tumours or leukaemias have shown a clear reduction in proliferation $[49,90-99]$. The reduction in cell proliferation has been explained by either increase in apoptosis or an arrest at the G0/G1 phase of cell cycle [49,90-99]. Nevertheless, the anti-tumour effects of blockers of $\mathrm{K}_{\mathrm{V}} 11.1$ appear to act through a reduction in cell proliferation $[49,82,98,99]$. Some studies have implicated the two isoforms of hERG (hERG1a and hERG1b) to play a vital role not only in cell proliferation 
by affecting different phases of cell cycle but also in the channel kinetics and current amplitude [100]. Both isoforms have been shown to coexist, but hERG1b expression is more prominent in the $S$ phase of the cell cycle and hERG1a expression in the G1 phase. Modulation of these expression patterns affects the cell proliferation [95]. Co-assembly of hERG1a with hERG1b results in increased availability of channels on the plasma membrane and a larger current flow when compared with homomeric forms of the channel [100]. Further insight into the hERG isoforms and its role in cancer is needed to conclusively designate hERG as a therapeutic target.

\section{7. $K_{\mathrm{y}} 10.1$}

$\mathrm{K}_{\mathrm{V}} 10.1$ (Eag1, encoded by KCNH1) is one of the best-studied ion channels in the context of cancer. Its oncogenic potential was first described in 1999 with the discovery that the inhibition of $\mathrm{K}_{\mathrm{V}} 10.1$ expression reduces proliferation of several somatic cancer cell lines [101]. $\mathrm{K}_{\mathrm{V}} 10.1$ overexpression, in turn, increases cell proliferation and can confer a transformed phenotype. In the same study, our laboratory also reported that $\mathrm{K}_{\mathrm{V}} 10.1$ is undetectable in healthy tissues outside the brain and favours xenograft tumour progression in immunodeficient mice in vivo. Along these lines, $\mathrm{K}_{\mathrm{V}} 10.1$ has also been detected in approximately $70 \%$ of human tumour biopsies of diverse origin [102-113]. Its widespread presence in clinical samples, together with the fact that the physiological expression of $\mathrm{K}_{\mathrm{V}} 10.1$ is confined to the brain (with the exception of a few restricted cell populations [111]), aroused a lot of interest in the channel owing to its potential therapeutic and diagnostic applications. It had been assumed that $\mathrm{K}_{\mathrm{V}} 10.1$ is present only in solid tumours but recent research has revealed its presence in leukaemias, correlating with a poor prognosis [107]. $\mathrm{K}_{\mathrm{V}} 10.1$ expression also correlates with poor prognosis for patients of ovarian [106], gastric [112] and colon cancer [114], and with lymph node metastasis in gastric cancer and head and neck squamous cell carcinoma, where it also correlates with the disease stage [105]. Moreover, a number of studies have supported the observation that $\mathrm{K}_{\mathrm{V}} 10.1$ blockage or knockdown decreases the proliferation of many cancer cell lines and in vivo tumour models $[53,107,115,116]$. An interesting exception here is glioblastoma, where the levels of $\mathrm{K}_{\mathrm{V}} 10.1$ are lower than that in healthy brain tissue [109], while further silencing of channel expression increases the responsiveness to interferon gamma treatment [117]. Although it is probably not the only relevant localization of $\mathrm{K}_{\mathrm{V}} 10.1$ [118], it is also worth mentioning here that membrane localization makes $\mathrm{K}_{\mathrm{V}} 10.1$ an attractive target for therapy, as it is easily accessible from the extracellular side. In order to selectively induce apoptosis in cancer cells, an anti- $\mathrm{K}_{\mathrm{V}} 10.1$ antibody has been coupled to TNF-related apoptosis-inducing ligand, and this strategy has been successfully tested in vitro [119].

The mechanisms of how $\mathrm{K}_{\mathrm{V}} 10.1$ is able to increase cell proliferation and favour tumour progression remain elusive. Ion permeation does not seem to be a necessary condition for either of the above, as non-conducting mutants retain the ability to influence proliferation and tumourigenesis [52,53]. By implication, the advantage $\mathrm{K}_{\mathrm{V}} 10.1$ expression confers is independent of the 'classical' contributions of $\mathrm{K}^{+}$ channels to proliferation: regulating cell volume, maintaining the driving force for $\mathrm{Ca}^{2+}$ and G1/S hyperpolarization. As we already indicated earlier, this is less surprising than it may appear, because if the features associated with $\mathrm{K}^{+}$permeation were enough to render a transformed phenotype, many more $\mathrm{K}^{+}$channels would be oncogenic. Moreover, the loss of ionic conductances can often be compensated for by other channels, which also does not fit into the picture where removing a particular conductance drastically reduces proliferation in so many cancer cell lines, as well as tumourigenesis in vivo. In contrast to ion permeation, voltage-dependent conformations may be crucial for $\mathrm{K}_{\mathrm{V}} 10.1$ to support proliferation, as the non-conducting mutants that have a preference for the open conformation fail to influence proliferation [52]. It is important to note that channel blockers could reduce proliferation not only by inhibiting permeation, but also by trapping the channel in a particular conformation. Hegle and co-workers also described an increase in p38-MAP kinase activity in non-cancer cells transfected with $K_{V} 10.1$, and abolishing the effect of $\mathrm{K}_{\mathrm{V}} 10.1$ on cell proliferation by $\mathrm{p}-38^{\mathrm{MAPK}}$ inhibition. Interestingly, modulation of $\mathrm{K}_{\mathrm{V}} 10.1$ expression levels by $\mathrm{p}-38^{\mathrm{MAPK}}$ pathway has been described in MG-63 cells from osteosarcoma [102], so the relation between the channel and p-38 $8^{\mathrm{MAPK}}$ signalling needs further clarification. Another non-conducting function of $K_{V} 10.1$ is an increase in hypoxia resistance by boosting HIF-1 levels and VEGF secretion, eventually leading to better tumour vascularization [53]. Nevertheless, the mechanisms described above remain insufficient to explain the benefit $\mathrm{K}_{\mathrm{V}} 10.1$ expression brings to the proliferation of so many different cancer cell lines.

Finally, in some models, $\mathrm{K}_{\mathrm{V}} 10.1$ appears to be regulated by cell cycle. Inducing the G2/M transition by progesterone in Xenopus oocytes heterologously expressing $\mathrm{K}_{\mathrm{V}} 10.1$ causes a reduction in current [17]. This reduction is dependent on the mitosis-promoting factor (MPF, a complex of cyclin B and $\mathrm{p} 34^{\mathrm{cdc} 2}$ ) and obeys a voltage-dependent block by intracellular $\mathrm{Na}^{+}$[16]. MPF induces an increase in selectivity during the $\mathrm{M}$ phase [120] that results in block by $\mathrm{Na}^{+}$, which leads to a rectification of the current-voltage relation. The resulting net loss of $\mathrm{K}^{+}$conductance at G2/M transition may be a way to achieve membrane depolarization associated with mitosis. Cell-cycle regulation of $\mathrm{K}_{\mathrm{V}} 10.1$ has also been studied in MCF-7 breast cancer cells. Synchronization of these cells in G0/G1 by serum starvation leads to an increase in Eag1 mRNA expression compared with unsynchronized controls, with a further increase during the progression through G1 and a decrease in the S-phase [121]. At the functional level, this is accompanied by an increase in outwardrectifier $\mathrm{K}^{+}$current that hyperpolarizes the membrane towards the S-phase [121]. Both $\mathrm{K}_{\mathrm{V}} 10.1$ mRNA and $\mathrm{K}_{\mathrm{V}} 10.1$ mediated current in MCF-7 cells can also be increased by stimulation with insulin-like growth factor 1 (IGF-1) via the PI3 K/Akt pathway, suggesting that the progression through G1 to S triggered by IGF-1 can partially be owing to its effect on $K_{V} 10.1$ [122]. Defective checkpoint control between G1 and S-phase can also result in $\mathrm{K}_{\mathrm{V}} 10.1$ upregulation. In SH-SY5Y neuroblastoma cells, $\mathrm{K}_{\mathrm{V}} 10.1$ expression is regulated by the p53/mir34/E2F1 pathway [123]. Additionally, keratinocytes immortalized with human papilloma virus oncogenes E6 and E7 targeting p53 and Retinoblastoma protein ( $\mathrm{pRb}$ ) start to transcribe $\mathrm{K}_{\mathrm{V}} 10.1 \mathrm{mRNA}$ [124]. One can thus expect that $\mathrm{p} 53$ or $\mathrm{pRb} / \mathrm{E} 2 \mathrm{~F}$ pathway inhibition or malfunctions, which are very common in cancer, can give rise to higher $\mathrm{K}_{\mathrm{V}} 10.1$ expression levels. However, further research is 
needed to establish that $\mathrm{K}_{\mathrm{V}} 10.1$ expression is cell-cycle dependent and to elucidate the effect(s) of the channel on cell-cycle progression.

\section{Conclusion}

Progression through the cell cycle is guarded by several checkpoint control pathways that have the ability to delay or stop further events, such as DNA synthesis or assembly of the mitotic spindle, before commitment into cell division. In accordance with the experimental data compiled in this review, there can be little doubt that $\mathrm{K}^{+}$channels play an active role in cell-cycle progression. On the other hand, their expression or function can be regulated by the cell cycle. Therefore, $\mathrm{K}^{+}$channels could also be viewed as effectors of the checkpoint machinery. As molecular machines that enable the passage of $\mathrm{K}^{+}$ions through the membrane, they can regulate cell volume, provide driving force for $\mathrm{Ca}^{2+}$ entry, hyperpolarize the cell at the G1/S transition and depolarize it towards mitosis. Additionally, non-canonical, permeation-independent mechanisms may be involved, where $\mathrm{K}^{+}$channels recruit or modulate signalling cascades via protein-protein interactions. It is tempting to assume that signalling cascades activated by such interactions could link the nuclear clock control with its cytoplasmic counterpart.

Unfortunately, to date we have only a rough estimate of how membrane potential changes along the cell cycle. Moreover, very little is known about the non-conducting functions of $\mathrm{K}^{+}$channels. Which signalling cascades can they modify? How do they interact with other proteins? There are also more general questions that remain unanswered. How exactly does membrane potential affect the cell-cycle machinery? Further research on $\mathrm{K}^{+}$channels in cell cycle and proliferation will give us better understanding of these fundamental processes and may have therapeutic implications.

\section{References}

1. Hartwell LH, Culotti J, Pringle JR, Reid BJ. 1974 Genetic control of the cell division cycle in yeast. Science 183, 46-51. (doi:10.1126/science.183. 4120.46)

2. Hartwell LH, Weinert TA. 1989 Checkpoints: controls that ensure the order of cell cycle events. Science 246, 629-634. (doi:10.1126/science.2683079)

3. Murray AW. 1992 Creative blocks: cell-cycle checkpoints and feedback controls. Nature 359, 599-604. (doi:10.1038/359599a0)

4. Foster DA, Yellen P, Xu L, Saqcena M. 2010 Regulation of $\mathrm{G} 1$ cell cycle progression: distinguishing the restriction point from a nutrientsensing cell growth checkpoint(s). Genes Cancer 1, 1124-1131. (doi:10.1177/1947601910392989)

5. Rieder CL. 2011 Mitosis in vertebrates: the G2/M and $M / A$ transitions and their associated checkpoints. Chromosome Res. 19, 291-306. (doi:10.1007/s10577-010-9178-z)

6. Blackiston DJ, McLaughlin KA, Levin M. 2009 Bioelectric controls of cell proliferation: ion channels, membrane voltage and the cell cycle. Cell cycle 8, 3519-3528. (doi:10.4161/cc.8. 21.9888)

7. Cone Jr CD. 1971 Unified theory on the basic mechanism of normal mitotic control and oncogenesis. J. Theor. Biol. 30, 151-181. (doi:10. 1016/0022-5193(71)90042-7)

8. Stillwell EF, Cone CM, Cone Jr CD. 1973 Stimulation of DNA synthesis in CNS neurones by sustained depolarisation. Nat. New Biol. 246, 110-111. (doi:10.1038/newbio246110a0)

9. Cone Jr CD, Cone CM. 1976 Induction of mitosis in mature neurons in central nervous system by sustained depolarization. Science 192, 155-158. (doi:10.1126/science.56781)

10. Boonstra J, Mummery CL, Tertoolen LG, Van Der Saag PT, De Laat SW. 1981 Cation transport and growth regulation in neuroblastoma cells: modulations of $\mathrm{K}^{+}$transport and electrical membrane properties during the cell cycle. J. Cell. Physiol. 107, 75-83. (doi:10.1002/jcp.1041070110)

11. Sachs HG, Stambrook PJ, Ebert JD. 1974 Changes in membrane potential during the cell cycle. Exp. Cell Res. 83, 362-366. (doi:10.1016/0014-4827(74) 90350-4)

12. Wonderlin WF, Woodfork KA, Strobl JS. 1995 Changes in membrane potential during the progression of MCF-7 human mammary tumor cells through the cell cycle. J. Cell. Physiol. 165, 177-185. (doi:10.1002/jcp.1041650121)

13. DeCoursey TE, Chandy KG, Gupta S, Cahalan MD. 1984 Voltage-gated $\mathrm{K}^{+}$channels in human T lymphocytes: a role in mitogenesis? Nature 307, 465-468. (doi:10.1038/307465a0)

14. Takahashi A, Yamaguchi H, Miyamoto H. 1993 Change in $\mathrm{K}^{+}$current of HeLa cells with progression of the cell cycle studied by patch-clamp technique. Am. J. Physiol. 265. C328-C336.

15. Arcangeli $A$, Bianchi $L$, Becchetti $A$, Faravelli $L$, Coronnello M, Mini E, Olivotto M, Wanke E. 1995 A novel inward-rectifying $\mathrm{K}^{+}$current with a cellcycle dependence governs the resting potential of mammalian neuroblastoma cells. J. Physiol. Lond. 489, 455-471.

16. Pardo LA, Bruggemann A, Camacho J, Stuhmer W. 1998 Cell cycle-related changes in the conducting properties of r-eag $\mathrm{K}^{+}$channels. J. Cell Biol. 143, 767-775. (doi:10.1083/jcb.143.3.767)

17. Bruggemann A, Stuhmer W, Pardo LA. 1997 Mitosis-promoting factor-mediated suppression of a cloned delayed rectifier potassium channel expressed in Xenopus oocytes. Proc. Natl Acad. Sci. USA 94, 537-542. (doi:10.1073/pnas.94.2.537)

18. Day ML, Pickering SJ, Johnson MH, Cook DI. 1993 Cell-cycle control of a large-conductance $\mathrm{K}^{+}$ channel in mouse early embryos. Nature $\mathbf{3 6 5}$, 560-562. (doi:10.1038/365560a0)

19. Becchetti A. 2011 lon channels and transporters in cancer. 1. Ion channels and cell proliferation in cancer. Am. J. Physiol. Cell Physiol. 301, C255-C265. (doi:10.1152/ajpcell.00047.2011)

20. Wonderlin WF, Strobl JS. 1996 Potassium channels, proliferation and G1 progression. J. Membr. Biol. 154, 91-107. (doi:10.1007/s002329900135)

21. Huang $X$ et al. 2012 Voltage-gated potassium channel EAG2 controls mitotic entry and tumor growth in medulloblastoma via regulating cell volume dynamics. Genes Dev. 26, 1780-1796. (doi:10.1101/gad.193789.112)

22. Chiu SY, Wilson GF. 1989 The role of potassium channels in Schwann cell proliferation in Wallerian degeneration of explant rabbit sciatic nerves. J. Physiol. 408, 199-222.

23. Chandy KG, DeCoursey TE, Cahalan MD, McLaughlin C, Gupta S. 1984 Voltage-gated potassium channels are required for human $\mathrm{T}$ lymphocyte activation. J. Exp. Med. 160, 369-385. (doi:10.1084/jem. 160.2.369)

24. Amigorena S, Choquet D, Teillaud JL, Korn H, Fridman WH. 1990 Ion channel blockers inhibit B cell activation at a precise stage of the $G 1$ phase of the cell cycle. Possible involvement of $\mathrm{K}^{+}$channels. J. Immunol. 144, 2038-2045.

25. Wilson GF, Chiu SY. 1993 Mitogenic factors regulate ion channels in Schwann cells cultured from newborn rat sciatic nerve. J. Physiol. 470, 501-520.

26. Fieber LA, Gonzalez DM, Wallace MR, Muir D. 2003 Delayed rectifier $K$ currents in NF1 Schwann cells. Pharmacological block inhibits proliferation. Neurobiol. Dis. 13, 136-146. (doi:10.1016/509699961(03)00031-7)

27. Prevarskaya N, Skryma R, Bidaux G, Flourakis M, Shuba Y. 2007 lon channels in death and differentiation of prostate cancer cells. Cell Death Differ. 14, 1295-1304. (doi:10.1038/sj.cdd. 4402162)

28. Villalonga $\mathrm{N}$, Ferreres $\mathrm{JC}$, Argiles $\mathrm{JM}$, Condom $\mathrm{E}$, Felipe A. 2007 Potassium channels are a new target field in anticancer drug design. Recent Patents 
Anticancer Drug Disc. 2, 212-223. (doi:10.2174/ 157489207782497181)

29. Arcangeli A, Crociani 0, Lastraioli E, Masi A, Pillozzi S, Becchetti A. 2009 Targeting ion channels in cancer: a novel frontier in antineoplastic therapy. Curr. Med. Chem. 16, 66-93. (doi:10.2174/ 092986709787002835)

30. Arcangeli A, Pillozzi S, Becchetti A. 2012 Targeting ion channels in leukemias: a new challenge for treatment. Curr. Med. Chem. 19, 683-696. (doi:10. 2174/092986712798992093)

31. Huber SM. 2013 Oncochannels. Cell Calcium 53, 241-255. (doi:10.1016/j.ceca.2013.01.001)

32. Lenzi D, Radke K, Wilson M. 1991 Clonal cells from embryonic retinal cell lines express qualitative electrophysiological differences. J. Neurobiol. 22, 823-836. (doi:10.1002/neu.480220804)

33. Lenzi D, Radke K, Wilson M. 1993 Symmetrical segregation of potassium channels at cytokinesis. J. Neurobiol. 24, 675-686. (doi:10.1002/neu. 480240511)

34. Day ML, Johnson MH, Cook DI. 1998 A cytoplasmic cell cycle controls the activity of a $\mathrm{K}^{+}$channel in pre-implantation mouse embryos. EMBO J. 17, 1952-1960. (doi:10.1093/emboj/17.7.1952)

35. Ciemerych MA. 1995 Chromatin condensation activity and cortical activity during the first three cell cycles of a mouse embryo. Mol. Reprod. Dev. 41, 416-424. (doi:10.1002/mrd.1080410404)

36. Ghiani $C A$, Yuan $X$, Eisen AM, Knutson PL, DePinho RA, McBain CJ, Gallo V. 1999 Voltage-activated K' channels and membrane depolarization regulate accumulation of the cyclin-dependent kinase inhibitors p27(Kip1) and p21(CIP1) in glial progenitor cells. J. Neurosci. 19, 5380-5392.

37. Chittajallu R, Chen $Y$, Wang $H$, Yuan $X$, Ghiani CA, Heckman T, McBain C, Gallo V. 2002 Regulation of Kv1 subunit expression in oligodendrocyte progenitor cells and their role in $\mathrm{G}(1) / S$ phase progression of the cell cycle. Proc. Natl Acad. Sci. USA 99, 2350-2355. (doi:10.1073/pnas. 042698399)

38. Doyle DA, Morais Cabral J, Pfuetzner RA, Kuo A, Gulbis JM, Cohen SL, Chait BT, MacKinnon R. 1998 The structure of the potassium channel: molecular basis of $\mathrm{K}^{+}$conduction and selectivity. Science $\mathbf{2 8 0}$, 69-77. (doi:10.1126/science.280.5360.69)

39. Sano $T$, Becker D, Ivashikina N, Wegner $L H$, Zimmermann U, Roelfsema MR, Nagata T, Hedrich R. 2007 Plant cells must pass a $\mathrm{K}^{+}$threshold to re-enter the cell cycle. Plant J. 50, 401-413. (doi:10.1111/j. 1365-313X.2007.03071.x)

40. Rouzaire-Dubois B, Dubois JM. 1991 A quantitative analysis of the role of $\mathrm{K}^{+}$channels in mitogenesis of neuroblastoma cells. Cell. Signal. 3, 333-339. (doi:10.1016/0898-6568(91)90062-Y)

41. Dubois JM, Rouzaire-Dubois B. 2004 The influence of cell volume changes on tumour cell proliferation. Eur. Biophys. J. 33, 227-232. (doi:10.1007/s00249003-0364-1)

42. Lee YS, Sayeed MM, Wurster RD. 1993 Inhibition of cell growth by $\mathrm{K}^{+}$channel modulators is due to interference with agonist-induced $\mathrm{Ca}^{2+}$ release. Cell.
Signal. 5, 803-809. (doi:10.1016/0898-6568(93) 90041-J)

43. Lin CS et al. 1993 Voltage-gated potassium channels regulate calcium-dependent pathways involved in human T lymphocyte activation. J. Exp. Med. 177, 637-645. (doi:10.1084/jem.177.3.637)

44. Lepple-Wienhues A, Berweck S, Bohmig M, Leo CP, Meyling B, Garbe C, Wiederholt M. $1996 \mathrm{~K}^{+}$ channels and the intracellular calcium signal in human melanoma cell proliferation. J. Membr. Biol. 151, 149-157. (doi:10.1007/5002329900066)

45. Lallet-Daher $\mathrm{H}$ et al. 2009 Intermediateconductance $\mathrm{Ca}^{2+}$-activated $\mathrm{K}^{+}$channels (IKCa1) regulate human prostate cancer cell proliferation through a close control of calcium entry. Oncogene 28, 1792-1806. (doi:10.1038/onc.2009.25)

46. Wang ZH, Shen B, Yao HL, Jia YC, Ren J, Feng YJ, Wang YZ. 2007 Blockage of intermediateconductance- $\mathrm{Ca}^{2+}$-activated $\mathrm{K}^{+}$channels inhibits progression of human endometrial cancer. Oncogene 26, 5107-5114. (doi:10.1038/sj.onc.1210308)

47. Jang SH et al. 2009 Silencing of Kv4.1 potassium channels inhibits cell proliferation of tumorigenic human mammary epithelial cells. Biochem. Biophys. Res. Commun. 384, 180-186. (doi:10.1016/j.bbrc. 2009.04.108)

48. Miguel-Velado E, Perez-Carretero FD, Colinas 0, Cidad P, Heras M, Lopez-Lopez JR, Perez-Garcia MT. 2010 Cell cycle-dependent expression of Kv3.4 channels modulates proliferation of human uterine artery smooth muscle cells. Cardiovasc. Res. 86, 383-391. (doi:10.1093/cvr/cvq011)

49. Glassmeier G, Hempel K, Wulfsen I, Bauer CK, Schumacher U, Schwarz JR. 2012 Inhibition of HERG1 $\mathrm{K}^{+}$channel protein expression decreases cell proliferation of human small cell lung cancer cells. Pflugers Arch. 463, 365-376. (doi:10.1007/s00424011-1045-z)

50. Yasuda T, Cuny H, Adams DJ. 2013 Kv3.1 channels stimulate adult neural precursor cell proliferation and neuronal differentiation. J. Physiol. 591, 2579 2591. (doi:10.1113/jphysiol.2012.249151)

51. Kaczmarek LK. 2006 Non-conducting functions of voltage-gated ion channels. Nat. Rev. Neurosci. 7, 761-771. (doi:10.1038/nrn1988)

52. Hegle AP, Marble DD, Wilson GF. 2006 A voltagedriven switch for ion-independent signaling by ether-a-go-go $\mathrm{K}^{+}$channels. Proc. Natl Acad. Sci. USA 103, 2886-2891. (doi:10.1073/pnas. 0505909103)

53. Downie BR, Sanchez A, Knotgen $H$, Contreras-Jurado C, Gymnopoulos M, Weber C, Stuhmer W, Pardo LA. 2008 Eag1 Expression Interferes with hypoxia homeostasis and induces angiogenesis in tumors. J. Biol. Chem. 283, 36 234-36 240. (doi:10.1074/ Jbc.M801830200)

54. Cidad P, Jimenez-Perez L, Garcia-Arribas D, MiguelVelado E, Tajada S, Ruiz-McDavitt C, Lopez-Lopez JR, Perez-Garcia MT. 2012 Kv1.3 channels can modulate cell proliferation during phenotypic switch by an ion-flux independent mechanism. Arterioscler. Thromb. Vasc. Biol. 32, 1299-1307. (doi:10.1161/ atvbaha.111.242727)
55. Millership JE, Devor DC, Hamilton KL, Balut $C M$, Bruce Jl, Fearon IM. 2011 Calcium-activated $\mathrm{K}^{+}$ channels increase cell proliferation independent of $\mathrm{K}^{+}$conductance. Am. J. Physiol. Cell Physiol. 300, C792-C802. (doi:10.1152/ajpcell.00274.2010)

56. Itoh T, Hasegawa J, Tsujita K, Kanaho Y, Takenawa T. 2009 The tyrosine kinase Fer is a downstream target of the PLD-PA pathway that regulates cell migration. Sci. Signal. 2, ra52. (doi:10.1126/scisignal.2000393)

57. Norring SA, Ednie AR, Schwetz TA, Du D, Yang H, Bennett ES. 2013 Channel sialic acids limit hERG channel activity during the ventricular action potential. FASEB J. 27, 622-631. (doi:10.1096/fj. 12-214387)

58. Lang F, Shumilina E. 2013 Regulation of ion channels by the serum- and glucocorticoid-inducible kinase SGK1. FASEB J. 27, 3-12. (doi:10.1096/jj. 12-218230)

59. Benson MD, Li QJ, Kieckhafer K, Dudek D, Whorton MR, Sunahara RK, Iniguez-Lluhi JA, Martens JR. 2007 SUM0 modification regulates inactivation of the voltage-gated potassium channel Kv1.5. Proc. Natl Acad. Sci. USA 104, 1805-1810. (doi:10.1073/ pnas.0606702104)

60. Cahalan MD, Chandy KG. 2009 The functional network of ion channels in T lymphocytes. Immunol. Rev. 231, 59-87. (doi:10.1111/j.1600-065X.2009. 00816.x)

61. Lam J, Wulff H. 2011 The lymphocyte potassium channels Kv1.3 and KCa3.1 as targets for immunosuppression. Drug Dev. Res. 72, 573-584. (doi:10.1002/ddr.20467)

62. Leonard RJ, Garcia ML, Slaughter RS, Reuben JP. 1992 Selective blockers of voltage-gated $\mathrm{K}^{+}$ channels depolarize human $T$ lymphocytes: mechanism of the antiproliferative effect of charybdotoxin. Proc. Natl Acad. Sci. USA 89, 10094 - 10 098. (doi:10.1073/pnas.89.21.10094)

63. Joiner WJ, Khanna R, Schlichter LC, Kaczmarek LK. 2001 Calmodulin regulates assembly and trafficking of SK4/IK1 $\mathrm{Ca}^{2+}$-activated $\mathrm{K}^{+}$channels. J. Biol. Chem. 276, 37 980-37985.

64. Hu J et al. 2007 Calcium-activated potassium channels mediated blood-brain tumor barrier opening in a rat metastatic brain tumor model. Mol. Cancer 6, 22. (doi:10.1186/1476-4598-6-22)

65. Bi D et al. 2013 The intermediate-conductance calcium-activated potassium channel KCa3.1 regulates vascular smooth muscle cell proliferation via controlling calcium-dependent signaling. J. Biol. Chem. 288, 15 843-15 853. (doi:10.1074/jbc.M112. 427187)

66. Su XL, Wang Y, Zhang W, Zhao LM, Li GR, Deng XL. 2011 Insulin-mediated upregulation of $K(C a) 3.1$ channels promotes cell migration and proliferation in rat vascular smooth muscle. J. Mol. Cell. Cardiol. 51, 51-57. (doi:10.1016/j.yjmcc.2011.03.014)

67. Wang SP, Wang JA, Luo RH, Cui WY, Wang H. 2008 Potassium channel currents in rat mesenchymal stem cells and their possible roles in cell proliferation. Clin. Exp. Pharmacol. Physiol. 35, 1077-1084. (doi:10.1111/j.1440-1681.2008. 04964.x)

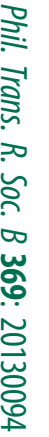


68. Yang XW, Liu JW, Zhang RC, Yin Q, Shen WZ, Yi JL. 2013 Inhibitory effects of blockage of intermediate conductance $\mathrm{Ca}^{2+}$-activated $\mathrm{K}^{+}$channels on proliferation of hepatocellular carcinoma cells. J. Huazhong Univ. Sci. Technol. Med. Sci. 33, 86-89. (doi:10.1007/s11596-013-1076-0)

69. Abdullaev IF, Rudkouskaya A, Mongin AA, Kuo YH. 2010 Calcium-activated potassium channels BK and IK1 are functionally expressed in human gliomas but do not regulate cell proliferation. PLOS ONE $\mathbf{5}$, e12304. (doi:10.1371/journal.pone.0012304)

70. Schwab A, Nechyporuk-Zloy V, Gassner B, Schulz C, Kessler W, Mally S, Romer M, Stock C. 2012 Dynamic redistribution of calcium sensitive potassium channels (hK(Ca)3.1) in migrating cells. J. Cell. Physiol. 227, 686-696. (doi:10.1002/ jcp.22776)

71. Schwab A, Reinhardt J, Schneider SW, Gassner B, Schuricht B. $1999 \mathrm{~K}^{+}$channel-dependent migration of fibroblasts and human melanoma cells. Cell. Physiol. Biochem. 9, 126-132. (doi:10.1159/ 000016309)

72. Schwab A, Wojnowski L, Gabriel K, Oberleithner H. 1994 Oscillating activity of a $\mathrm{Ca}^{2+}$-sensitive $\mathrm{K}^{+}$ channel. A prerequisite for migration of transformed Madin-Darby canine kidney focus cells. J. Clin. Investig. 93, 1631-1636. (doi:10.1172/JC1117144)

73. Schwab A et al. 2006 Subcellular distribution of calcium-sensitive potassium channels (IK1) in migrating cells. J. Cell. Physiol. 206, 86-94. (doi:10.1002/jcp.20434)

74. Catacuzzeno L, Aiello F, Fioretti B, Sforna L, Castigli E, Ruggieri P, Tata AM, Calogero A, Franciolini F. 2011 Serum-activated $\mathrm{K}$ and $\mathrm{Cl}$ currents underlay U87-MG glioblastoma cell migration. J. Cell. Physiol. 226, 1926-1933. (doi:10.1002/jcp.22523)

75. Ruggieri P et al. 2012 The inhibition of KCa3.1 channels activity reduces cell motility in glioblastoma derived cancer stem cells. PLOS ONE 7, e47825. (doi:10.1371/journal.pone.0047825)

76. Kotecha SA, Schlichter LC. 1999 A Kv1.5 to Kv1.3 switch in endogenous hippocampal microglia and a role in proliferation. J. Neurosci. 19, $10680-10693$.

77. Fordyce CB, Jagasia R, Zhu X, Schlichter LC. 2005 Microglia Kv1.3 channels contribute to their ability to kill neurons. J. Neurosci. 25, 7139-7149. (doi:10. 1523/jneurosci.1251-05.2005)

78. Villalonga N, David M, Bielanska J, Gonzalez T, Parra D, Soler C, Comes N, Valenzuela C, Felipe A. 2010 Immunomodulatory effects of diclofenac in leukocytes through the targeting of Kv1.3 voltagedependent potassium channels. Biochem. Pharmacol. 80, 858-866. (doi:10.1016/j.bcp.2010. 05.012)

79. Villalonga N, Escalada A, Vicente R, Sanchez-Tillo E, Celada A, Solsona C, Felipe A. 2007 Kv1.3/Kv1.5 heteromeric channels compromise pharmacological responses in macrophages. Biochem. Biophys. Res. Commun. 352, 913-918. (doi:10.1016/j.bbrc.2006. 11.120)

80. Vicente $R$ et al. 2003 Differential voltage-dependent $\mathrm{K}^{+}$channel responses during proliferation and activation in macrophages. J. Biol. Chem. 278, 46 307-46 320. (doi:10.1074/jbc.M304388200)

81. Erdogan A et al. 2005 Margatoxin inhibits VEGFinduced hyperpolarization, proliferation and nitric oxide production of human endothelial cells. J. Vasc. Res. 42, 368-376. (doi:10.1159/ 000087159)

82. Li H, Liu L, Guo L, Zhang J, Du W, Li X, Liu W, Chen $X$, Huang S. 2008 HERG $\mathrm{K}^{+}$channel expression in $\mathrm{CD}^{+} / 4^{+} / \mathrm{CD} 38^{-} / \mathrm{CD} 123$ (high) cells and primary leukemia cells and analysis of its regulation in leukemia cells. Int. J. Hematol. 87, 387-392. (doi:10.1007/s12185-008-0056-9)

83. Moudgil R, Michelakis ED, Archer SL. 2006 The role of $\mathrm{K}^{+}$channels in determining pulmonary vascular tone, oxygen sensing, cell proliferation, and apoptosis: implications in hypoxic pulmonary vasoconstriction and pulmonary arterial hypertension. Microcirculation 13, 615-632. (doi:10.1080/10739680600930222)

84. Tian Y, Yue X, Luo D, Wazir R, Wang J, Wu T, Chen L, Liao B, Wang K. 2013 Increased proliferation of human bladder smooth muscle cells is mediated by physiological cyclic stretch via the PI3KSGK1Kv1.3 pathway. Mol. Med. Rep. 8, 294-298. (doi:10.3892/ mmr.2013.1473)

85. Cheong A et al. 2011 Potent suppression of vascular smooth muscle cell migration and human neointimal hyperplasia by $\mathrm{K}_{\mathrm{v}} 1.3$ channel blockers. Cardiovasc. Res. 89, 282-289. (doi:10.1093/cvr/ cvq305)

86. Warmke JW, Ganetzki B. 1994 A family of potassium channel genes related to eag in Drosophila and mammals. Proc. Natl Acad. Sci. USA 91, 3438-3442. (doi:10.1073/pnas.91.8.3438)

87. Pond AL, Scheve BK, Benedict AT, Petrecca K, Van Wagoner DR, Shrier A, Nerbonne JM. 2000 Expression of distinct ERG proteins in rat, mouse, and human heart - Relation to functional I-Kr channels. J. Biol. Chem. 275, 5997-6006. (doi:10. 1074/jbc.275.8.5997)

88. Rosati B, Marchetti P, Crociani 0, Lecchi M, Lupi R, Arcangeli A, Olivotto M, Wanke E. 2000 Glucoseand arginine-induced insulin secretion by human pancreatic beta-cells: the role of HERG $\mathrm{K}^{+}$channels in firing and release. FASEB J. 14, 2601-2610. (doi:10.1096/fj.00-0077com)

89. Cherubini $A$ et al. 2000 HERG potassium channels are more frequently expressed in human endometrial cancer as compared to non-cancerous endometrium. Br. J. Cancer 83, 1722-1729. (doi:10.1054/bjoc.2000.1497)

90. Pillozzi $S$ et al. 2002 HERG potassium channels are constitutively expressed in primary human acute myeloid leukemias and regulate cell proliferation of normal and leukemic hemopoietic progenitors. Leukemia 16, 1791-1798. (doi:10.1038/sj. leu.2402572)

91. Lastraioli E et al. 2004 herg1 gene and HERG1 protein are overexpressed in colorectal cancers and regulate cell invasion of tumor cells. Cancer Res. 64, 606-611. (doi:10.1158/0008-5472. (AN-03-2360)
92. Ding XW, Yang WB, Gao S, Wang W, Li Z, Hu WM, Li JJ, Luo HS. 2010 Prognostic significance of hERG1 expression in gastric cancer. Dig. Dis. Sci. 55, 1004-1010. (doi:10.1007/s10620-009-0834-0)

93. Shao XD, Wu KC, Guo XZ, Xie MJ, Zhang J, Fan DM. 2008 Expression and significance of HERG protein in gastric cancer. Cancer Biol. Ther. 7, 45-50. (doi:10. 4161/cbt.7.1.5126)

94. Smith GAM, Tsui HW, Newell EW, Jiang XP, Zhu XP, Tsui FWL, Schlichter LC. 2002 Functional upregulation of HERG $\mathrm{K}^{+}$channels in neoplastic hematopoietic cells. J. Biol. Chem. 277, $18528-$ 18 534. (doi:10.1074/jbc.M200592200)

95. Crociani 0, Guasti L, Balzi M, Becchetti A, Wanke E, Olivotto M, Wymore RS, Arcangeli A. 2003 Cell cycle-dependent expression of HERG1 and HERG1B isoforms in tumor cells. J. Biol. Chem. 278, 2947-2955. (doi:10.1074/jbc.M210789200)

96. Wang H, Zhang Y, Cao L, Han H, Wang J, Yang B, Nattel S, Wang Z. 2002 HERG K ${ }^{+}$channel, a regulator of tumor cell apoptosis and proliferation. Cancer Res. 62, 4843-4848.

97. Zhao J, Wei XL, Jia YS, Zheng JQ. 2008 Silencing of herg gene by shRNA inhibits SH-SY5Y cell growth in vitro and in vivo. Eur. J. Pharmacol. 579, 50-57. (doi:10.1016/j.jphar.2007.10.008)

98. Afrasiabi E, Hietamaki M, Viitanen T, Sukumaran P, Bergelin N, Tornquist K. 2010 Expression and significance of HERG (KCNH2) potassium channels in the regulation of MDA-MB-435S melanoma cell proliferation and migration. Cell. Signal. 22, 57-64. (doi:10.1016/j.cellsig.2009.09.010)

99. Cui G, Shu W, Wu Q, Chen Y. 2009 Effect of Gambogic acid on the regulation of $h E R G$ channel in K562 cells in vitro. J. Huazhong Univ. Sci. Technol. Med. Sci. 29, 540-545. (doi:10.1007/s11596-0090503-8)

100. Guasti L et al. 2008 Identification of a posttranslational mechanism for the regulation of hERG1 $\mathrm{K}^{+}$channel expression and hERG1 current density in tumor cells. Mol. Cell. Biol. 28, 5043-5060. (doi:10.1128/MCB.00304-08)

101. Pardo LA, del Camino D, Sanchez A, Alves F, Bruggemann A, Beckh S, Stuhmer W. 1999 Oncogenic potential of EAG K+ channels. EMBO J. 18, 5540-5547. (doi:10.1093/emboj/18.20.5540)

102. Wu X, Zhong D, Lin B, Zhai W, Ding Z, Wu J. 2013 p38 MAPK regulates the expression of ether a gogo potassium channel in human osteosarcoma cells. Radiol. Oncol. 47, 42-49. (doi:10.2478/v10019012-0043-x)

103. del Pliego MG et al. 2013 Expression of Eag1 $\mathrm{K}^{+}$ channel and ErbBs in human pituitary adenomas: cytoskeleton arrangement patterns in cultured cells. Int. J. Clin. Exp. Pathol. 6, 458-468.

104. Bai Y, Liao H, Liu T, Zeng X, Xiao F, Luo L, Guo H, Guo L. 2013 MiR-296-3p regulates cell growth and multi-drug resistance of human glioblastoma by targeting ether-a-go-go (EAG1). Eur. J. Cancer 49, 710-724. (doi:10.1016/j.ejca.2012.08.020)

105. Menendez ST et al. 2012 Frequent aberrant expression of the human ether a go-go (hEAG1) potassium channel in head and neck cancer: 
pathobiological mechanisms and clinical implications. J. Mol. Med. 90, 1173-1184. (doi:10. 1007/500109-012-0893-0)

106. Asher V, Khan R, Warren A, Shaw R, Schalkwyk GV, Bali A, Sowter HM. 2010 The Eag potassium channel as a new prognostic marker in ovarian cancer. Diagn. Pathol. 5, 78. (doi:10.1186/1746-1596-5-78)

107. Agarwal JR, Griesinger F, Stuhmer W, Pardo LA. 2010 The potassium channel Ether a go-go is a novel prognostic factor with functional relevance in acute myeloid leukemia. Mol Cancer 9, 18. (doi:10. 1186/1476-4598-9-18)

108. Asher V, Sowter H, Shaw R, Bali A, Khan R. 2010 Eag and HERG potassium channels as novel therapeutic targets in cancer. World J. Surg. Oncol. 8, 113. (doi:10.1186/1477-7819-8-113)

109. Patt S, Preussat K, Beetz C, Kraft R, Schrey M, Kalff R, Schonherr K, Heinemann SH. 2004 Expression of ether a go-go potassium channels in human gliomas. Neurosci. Lett. 368, 249-253. (doi:10. 1016/j.neulet.2004.07.001)

110. de Queiroz FM, Suarez-Kurtz G, Stuhmer W, Pardo LA. 2006 Ether a go-go potassium channel expression in soft tissue sarcoma patients. Mol. Cancer 5, 42. (doi:10.1186/1476-4598-5-42)

111. Hemmerlein B et al. 2006 Overexpression of Eagl potassium channels in clinical tumours. Mol Cancer 5, 42. (doi:10.1186/1476-4598-5-41)

112. Ding XW, Luo HS, Jin X, Yan JJ, Ai YW. 2007 Aberrant expression of Eag1 potassium channels in gastric cancer patients and cell lines. Med. Oncol. 24, 345-350. (doi:10.1007/s12032-007-0015-y)

113. Ding XW, Yan JJ, An P, Lu P, Luo HS. 2007 Aberrant expression of ether a go-go potassium channel in colorectal cancer patients and cell lines. World J. Gastroenterol. 13, 1257-1261.

114. Ousingsawat J, Spitzner $M$, Puntheeranurak $S$, Terracciano L, Tornillo L, Bubendorf L, Kunzelmann K, Schreiber R. 2007 Expression of voltage-gated potassium channels in human and mouse colonic carcinoma. Clin. Cancer Res. 13, 824-831. (doi:10. 1158/1078-0432.ccr-06-1940)

115. Gomez-Varela D et al. 2007 Monoclonal antibody blockade of the human Eag1 potassium channel function exerts antitumor activity. Cancer Res. 67, 7343-7349. (doi:10.1158/0008-5472.Can-07-0107)

116. Weber C, de Queiroz FM, Downie BR, Suckow A, Stuhmer W, Pardo LA. 2006 Silencing the activity and proliferative properties of the human Eagl potassium channel by RNA interference. J. Biol. Chem. 281, 13030 - 13 037. (doi:10.1074/Jbc. M600883200)

117. Cunha LC, Del Bel E, Pardo L, Stuhmer W, Titze DEAR. 2013 RNA interference with EAG1 enhances interferon gamma injury to glioma cells in vitro. Anticancer Res. 33, 865-870.

118. Chen $Y$, Sanchez A, Rubio ME, Kohl T, Pardo LA, Stuhmer W. 2011 Functional K(v)10.1 channels localize to the inner nuclear membrane. PLOS ONE 6, e19257. (doi:10.1371/journal.pone.0019257)
119. Hartung F, Stuhmer W, Pardo LA. 2011 Tumor cellselective apoptosis induction through targeting of K(V) 10.1 via bifunctional TRAIL antibody. Mol. Cancer 10, 109. (doi:10.1186/1476-4598-10-109)

120. Camacho J, Sanchez A, Stuhmer W, Pardo LA. 2000 Cytoskeletal interactions determine the electrophysiological properties of human EAG potassium channels. Pflugers Arch. Eur. J. Phys. 441, 167-174. (doi:10.1007/s004240000420)

121. Ouadid-Ahidouch $H$, Le Bourhis $X$, Roudbaraki $M$, Toillon RA, Delcourt P, Prevarskaya N. 2001 Changes in the $\mathrm{K}^{+}$current-density of MCF-7 cells during progression through the cell cycle: possible involvement of a h-ether.a-gogo $\mathrm{K}^{+}$channel. Receptors Channels 7, 345-356.

122. Borowiec AS, Hague $F$, Harir $N$, Guenin $S$, Guerineau F, Gouilleux F, Roudbaraki M, Lassoued K, OuadidAhidouch H. 2007 IGF-1 activates hEAG K ${ }^{+}$channels through an Akt-dependent signaling pathway in breast cancer cells: role in cell proliferation. J. Cell. Physiol. 212, 690-701. (doi:10.1002/jcp.21065)

123. Lin $H$, Li Z, Chen C, Luo X, Xiao J, Dong D, Lu Y, Yang B, Wang Z. 2011 Transcriptional and posttranscriptional mechanisms for oncogenic overexpression of ether a go-go $\mathrm{K}^{+}$channel. PLoS ONE 6, e20362. (doi:10.1371/journal.pone.0020362)

124. Diaz L et al. 2009 Estrogens and human papilloma virus oncogenes regulate human ether-a-go-go-1 potassium channel expression. Cancer Res. 69, 3300-3307. (doi:10.1158/0008-5472.can-08-2036)
9 
ARTICLE

Received 25 Jul 2014 | Accepted 18 Feb 2015 | Published 30 Mar 2015

DOI: $10.1038 /$ ncomms 7672

OPEN

\section{Voltage-dependent gating of $\mathrm{KCNH}$ potassium channels lacking a covalent link between voltage-sensing and pore domains}

Éva Lörinczi ${ }^{1, *, \dagger}$, Juan Camilo Gómez-Posadaa ${ }^{1, \star}$, Pilar de la Peña ${ }^{2, \star}$, Adam P. Tomczak ${ }^{3}$, Jorge Fernández-Trillo ${ }^{3}$, Ulrike Leipscher ${ }^{3}$, Walter Stühmer ${ }^{1,4}$, Francisco Barros ${ }^{2, \star} \&$ Luis A. Pardo ${ }^{3, *}$

Voltage-gated channels open paths for ion permeation upon changes in membrane potential, but how voltage changes are coupled to gating is not entirely understood. Two modules can be recognized in voltage-gated potassium channels, one responsible for voltage sensing (transmembrane segments S1 to S4), the other for permeation (S5 and S6). It is generally assumed that the conversion of a conformational change in the voltage sensor into channel gating occurs through the intracellular S4-S5 linker that provides physical continuity between the two regions. Using the pathophysiologically relevant $\mathrm{KCNH}$ family, we show that truncated proteins interrupted at, or lacking the S4-S5 linker produce voltage-gated channels in a heterologous model that recapitulate both the voltage-sensing and permeation properties of the complete protein. These observations indicate that voltage sensing by the S4 segment is transduced to the channel gate in the absence of physical continuity between the modules.

\footnotetext{
${ }^{1}$ Department of Molecular Biology of Neuronal Signals, Max Planck Institute of Experimental Medicine, Hermann-Rein-Straße 3, 37075 Göttingen, Germany.

${ }^{2}$ Departamento de Bioquímica y Biología Molecular, Universidad de Oviedo, Edificio Santiago Gascón, Campus de El Cristo, 33006 Oviedo, Spain.

${ }^{3}$ Oncophysiology Group, Max Planck Institute of Experimental Medicine, Hermann-Rein-Straße 3, 37075 Göttingen, Germany. ${ }^{4}$ Cluster of Excellence Nanoscale Microscopy and Molecular Physiology of the Brain, 37073 Göttingen, Germany. ${ }^{\star}$ These authors contributed equally to this work. $\dagger$ Present address: Department of Cell Physiology and Pharmacology, University of Leicester, Leicester LE1 9HN, UK. Correspondence and requests for materials should be addressed to F.B. (email: fbarros@uniovi.es) or to L.A.P. (email: pardo@em.mpg.de).
} 
$\mathrm{V}$ oltage-gated potassium $\left(\mathrm{K}_{\mathrm{V}}\right)$ channels are crucial regulators of cell excitability. They allow potassium to flow along its electrochemical gradient upon depolarization of the plasma membrane. Similar to voltage-gated $\mathrm{Na}^{+}$and $\mathrm{Ca}^{2+}$ channels, $\mathrm{K}_{\mathrm{V}}$ channels have four-fold symmetry that is generated by internal repeats in $\mathrm{Na}^{+}$and $\mathrm{Ca}^{2+}$ channels and by independent subunits in $\mathrm{K}^{+}$channels. Thus, $\mathrm{K}_{\mathrm{V}}$ channels are tetramers, each monomer containing six transmembrane segments (S1 to S6). A modular structure can be recognized in the arrangement of these channels. Functional $\mathrm{K}_{\mathrm{V}}$ channels are formed by an ensemble of a 'permeation module' or pore domain that allows potassium flow, constituted by four S5 and S6 segments and their intervening pore loop, and that is surrounded by a 'voltage-sensing module' comprised of four functionally independent voltage-sensor domains ${ }^{1-3}$. The permeation module would be equivalent to the two transmembrane segments of the bacterial KcsA or of the inward rectifier channels, which have little or no intrinsic voltage dependence (reviewed by Vardanyan and Pongs ${ }^{4}$ ). Voltage dependence is conferred by the rest of the transmembrane protein (segments S1-S4, although intracellular parts or even additional proteins can participate and modulate the process (see, for example, refs 5,6). Within the voltage-sensing domain, S4 shows an array of positive charges that drive its movement upon changes in voltage across the membrane $e^{2,3,7}$. Periodically distributed positively charged amino-acid residues at the S4 segment sense changes in the electric field across the membrane, and this stimulus is transduced into a conformational change in the permeation module that finally allows ion flow ${ }^{4}$.

The mechanism responsible for converting the changes occurring in the 'voltage-sensing module' into an opening of the gate of the 'permeation module' is not well understood. Current knowledge indicates that the intracellular linker between S4 and S5 segments is crucial for the so-called 'electromechanical coupling' in potassium channels by forming a rigid $\alpha$-helical connection 8,9 between the voltage sensor and the pore module ${ }^{10,11}$ that acting as a mechanical lever ${ }^{12-14}$ exerts force on the bottom part of S6, constituting the structural link coupling voltage sensing to channel gating ${ }^{4,15}$. Most evidence stems from channels of the families KCNA to $D$, although some studies have also been performed on HERG (KCNH2, $\left.\mathrm{K}_{\mathrm{V}} 11.1\right)$ (refs 10,16-18). In this case, it has been proposed that an electrostatic interaction between the S4-S5 linker and the bottom part of S6 stabilizes the closed conformation, and that outward S4 movement should pull on the linker, subsequently pulling also the $S 6$ bottom resulting in pore opening $10,19,20$.

We decided to test the relevance of the S4-S5 linker in channels of the $\mathrm{KCNH}$ family $\left(\mathrm{K}_{\mathrm{V}} 10.1, \mathrm{~K}_{\mathrm{V}} 10.2\right.$ and $\left.\mathrm{K}_{\mathrm{V}} 11.1\right)$ for several reasons. Although these channels lack the PVP motif at the end of S6 that induces a kink that allows channel gating in classical Shaker-like $\mathrm{K}_{\mathrm{V}}$ channels ${ }^{21}$, trapping of high- and lowaffinity blockers upon channel closing 22,23 and cysteine and proline scans of the S6 segment ${ }^{24,25}$, indicate that in $\mathrm{K}_{\mathrm{V}} 11.1$ channels the main ion conduction gate ('activation gate') is also formed by the bundle crossing of the inner portion of the four pore domain helices. In addition, $\mathrm{KCNH}$ channels share a relatively negative activation threshold and are open at $0 \mathrm{mV}$, raising the possibility that coupling between the voltage-sensing and permeation modules may be required to maintain the channel closed, rather than to open the gate. Finally, $\mathrm{K}_{\mathrm{V}} 10.1$ (KCNH1, Eag1) offers an interesting model for the study of voltage-dependent gating, because its activation is slow, permitting relatively easy characterization, but also because the time constant of activation depends very strongly on the prepulse potential and is modulated by extracellular magnesium ${ }^{26}$ allowing an unequivocal identification of its currents. Moreover, the activation/inactivation properties of $\mathrm{K}_{\mathrm{V}} 11.1$ and the implication of different regions of the channel in determining such properties have been studied in great detail, because its peculiar kinetic properties are crucial for the physiological role of $\mathrm{K}_{\mathrm{V}} 11.1$ during the cardiac action potential ${ }^{27}$.

In this report, we demonstrate that at least for $\mathrm{KCNH}$ channels, the physical continuity between the voltage sensing and the pore modules is not necessary for voltage-dependent gating, challenging the classical view of a S4-S5 linker acting as a rigid mechanical coupler between them, and opening new questions about the nature of the molecular and functional interactions between the voltage-sensing and pore modules of the protein.

\section{Results}

Voltage-gated currents in channels split at the S4-S5 linker. To study the requirement of the integrity of the S4-S5 linker, we introduced a stop codon in $\mathrm{K}_{\mathrm{V}} 10.1$ after each residue in the linker (341-349: sequence LDHYIEYGA) and removed the rest of the channel sequence. The truncated proteins were expressed in Xenopus oocytes in combination with the carboxyl-terminal (C-terminal) half of the channel, generated by removal of the initial coding sequence and positioning a start codon at the beginning of S5 (before residues 342 to 350 , see schematic representation in Fig. 1a). The resulting constructs were transcribed in vitro and combinations of two RNAs (encoding together the whole sequence interrupted at each of the positions: for example, 1-347 with 348-962) were co-injected into oocytes. None of the tested constructs gave rise to current when injected alone (Fig. 1d; $>20$ oocytes measured of each type in at least five independent injections). In contrast, when any of the combinations of interrupted amino $(\mathrm{N})$ - and $\mathrm{C}$-terminal truncated proteins were expressed together (as split channels ${ }^{28}$ ), it was possible to measure voltage-dependent potassium currents. Current amplitudes were smaller in split than in continuous channels (for example, at $+40 \mathrm{mV}, 12.26 \pm 1.27 \mu \mathrm{A}$, mean \pm s.e., $n=29$ in the split versus $21.55 \pm 4.58 \mu \mathrm{A}$, mean \pm s.e. $n=27$ for the wild type, when injecting 1 ng RNA), but the difficulties in adjusting RNA concentration per oocyte when injecting two different species at the same time and the requirement of longer incubation periods for robust expression of the split channels prevented a systematic study of crude current amplitudes. Representative current traces of the split channel interrupted after Y347 are shown in Fig. 1b,c in 2.5 and $60 \mathrm{mM}$ extracellular $\mathrm{K}^{+}$, respectively, and its voltage dependence is shown in Fig. 1e. The conductance/voltage plots were obtained through extrapolation to time zero of a double exponential fit of the tail current obtained in the presence of $60 \mathrm{mM} \mathrm{K}^{+}$in the external solution (substituting $60 \mathrm{mM} \mathrm{Na}^{+}$) as indicated by the corresponding symbols in Fig. 1c. The semi-maximal activation was shifted in the split channel to less depolarized potentials $(11.9 \pm 0.67$-split, mean \pm s.d., $n=8$ - versus $16.3 \pm 0.58 \mathrm{mV}$ -wild type, mean \pm s.e., $n=13-)$ and the slope was shallower (26.2 \pm 0.46 versus $20.5 \pm 0.30 \mathrm{mV}$, mean \pm s.e.). The differences were nevertheless not dramatic, indicating that the combination of the two independent demi-channels results in a functional, voltage-gated ion channel with properties similar to the continuous protein.

Our approach allowed us to combine any of the N-terminal halves with the C-terminal demi-channel starting at position 350, generating de facto deletions of 1 to 9 residues in the linker. In the case of the longest deletion (co-expression of demi-channels 1-341 and 350-962), the complex would eventually have no S4-S5 linker. When injected into oocytes, every combination of constructs gave rise to voltage-dependent currents consolidating the concept of a functional coupling between the voltage-sensing module and the permeation module in the absence of physical continuity. This was also true for the split channel completely 
a

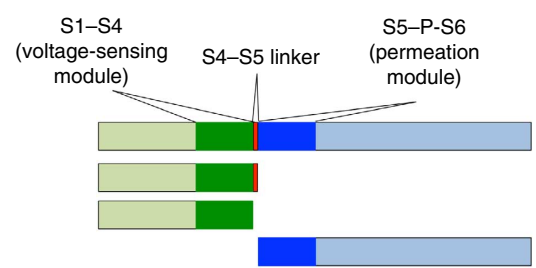

b
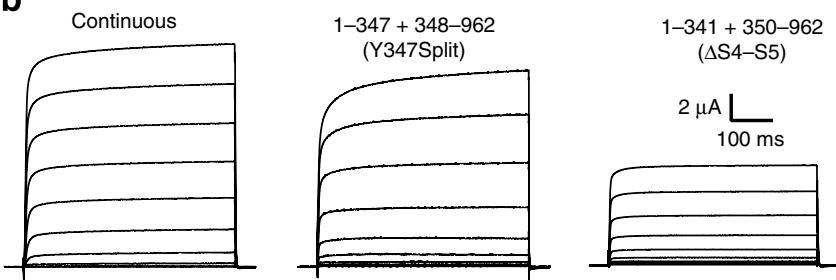

C

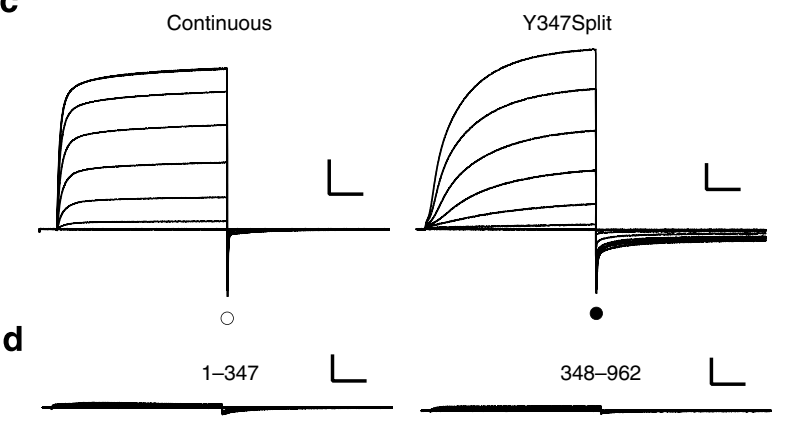

e

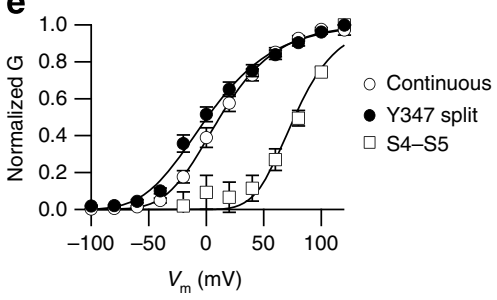

Figure 1 | Discontinuous $K_{\mathbf{v}} 10.1$ channels give rise to voltage-dependent currents. (a) Schematic representation of $\mathrm{K}_{\mathrm{v}} 10.1$ deletions. The voltagesensing domain is depicted in green, the $\mathrm{S} 4-\mathrm{S} 5$ linker in red and the pore module in dark blue. (b) Families of current traces obtained by depolarizations to different potential (up to $+80 \mathrm{mV}$ at $20 \mathrm{mV}$ intervals) from a holding voltage of $-80 \mathrm{mV}$ in oocytes injected with cRNA for continuous $\mathrm{K}_{\mathrm{v}} 10.1$ (left), a mixture of cRNAs encoding for residues 1-347 and 348-962 (centre) or residues 1-341 and 350-962 (right). All gave rise to outward currents with similar kinetics, but with some differences in their voltage dependence as illustrated in e. Extracellular solution containing $2.5 \mathrm{mM} \mathrm{KCl}$ and no $\mathrm{MgCl}_{2}$ was used. (c). Representative families of currents for continuous and 347-split $K_{v} 10.1$ channels recorded in $60 \mathrm{mM}$ extracellular $\mathrm{K}^{+}$. The tail amplitude at time 0 (represented in e) was extrapolated from biexponential fits as described in Methods and as indicated by symbols. (d) Families of currents elicited in $60 \mathrm{mM}$ extracellular $\mathrm{K}^{+}$using oocytes injected with cRNA encoding 1-347 (left) or 348-962 (right) $K_{\mathrm{V}} 10.1$ demi-channels. Note the lack of currents after injection of each of the demi-channels alone as compared with the continuous and the split channels (lower panels; scale bars, $2 \mu \mathrm{A}, 100 \mathrm{~ms}$ ). (e). Conductance/voltage plot of continuous (open circles, $n=13$ ), 347split (closed circles, $n=12$ ) and split $K_{V} 10.1$ channels lacking the S4-S5 linker (open squares, $n=11$ ). The currents elicited by channels completely lacking the S4-S5 segment required stronger depolarizations to develop, but the slope (that is, voltage dependence) was not dramatically different. Error bars represent s.e. lacking a S4-S5 linker (Fig. 1b). Although in this case the threshold for activation was strongly shifted to depolarized potentials (Fig. 1e), and the $I / V$ curves did not reach saturation in the voltage range tested, precluding an accurate estimation of voltage-dependence parameters, there was unequivocal voltage dependence. The activation of this construct was also remarkably slower than wild type, and therefore the accurate estimation of conductance at moderate depolarizations would require very long stimuli. This lack of accuracy at low depolarizations could explain the apparent biphasic behaviour of this construct, but we have not further studied this property.

Demi-channels coexist at the plasma membrane of oocytes. Our results unequivocally show that co-expression of two independent RNAs each encoding a 'demi-channel' give rise to voltage-gated currents. This can be due to the assembly of the two independent proteins into multimers that recapitulate properties of the continuous channel, but it could also be possible that expression of both fragments induce the functional expression of an otherwise not detected endogenous voltage-gated channel. However, this cannot be due to only one of the fragments, since we did not observe either outward or tail currents upon injection of any of the demi-channels alone (Fig. 1d). Interestingly, surface biotinylation experiments (Fig. 2a) combined with immunoblot using a polyclonal anti- $\mathrm{K}_{\mathrm{V}} 10.1 \mathrm{C}$-terminal antibody to detect only protein exposed to the extracellular medium revealed that the C-terminal demi-channel is indeed expressed on the surface of injected oocytes, not only when injected together with the $\mathrm{N}$-terminal part (second lane in Fig. 2a), but also when injected alone (third lane). As stated below, the results with non-conducting mutant demi-channels also indicate that the currents elicited by co-expression of both channel halves are not due to an endogenous oocyte channel.

To confirm that the two demi-channels actually co-assemble into a single complex and are still independent proteins, we tagged each of them using $5 \times$ Myc (on the $\mathrm{N}$ terminus) or a $4 \times \mathrm{HA}$ tags (C-terminal) to perform cross pull-down experiments. Pull down of HA gave a signal in Myc immunoblots (Fig. 2b) and vice versa, pull down of the N-terminal fragment (Myc) gave a positive signal in HA western blot (Fig. 2c). In both cases, the pulled-down fragments had the expected size $(\sim 50 \mathrm{kDa}$ for the $\mathrm{N}$-terminal and $\sim 70 \mathrm{kDa}$ for the C-terminal demichannels), indicating that the two halves are not covalently bound in the oocyte. The full-length protein carries sugar moieties in the S5-S6 linker, close to the pore ${ }^{29}$. To test if the C-terminal fragment (HA), which contains the glycosylation sites, could also be glycosylated in the split channels, we performed enzymatic deglycosylation of oocyte extracts (Fig. 2c). Treatment with PNGase F induced changes in the electrophoretic mobility of the HA-positive band, compatible with complex glycosylation of the truncated protein. The same treatment did not induce changes in the migration pattern of the band detected by Myc, which is not expected to carry glycosylation (Fig. 2b).

Finally, when extracts from oocytes were run under native conditions, co-expression of the two fragments gave rise to a large complex, with migration similar to the full-length channel (Fig. 2d). This again indicates that both fragments are expressed and share the same complex.

In summary, we conclude that upon expression of the channel fragments, a functional complex is formed by the apposition of voltage sensors and permeation modules that gives rise to a functional, voltage-dependent ion channel.

Split channels retain the properties of both functional modules. If the demi-channels indeed form a voltage-gated complex, 




b
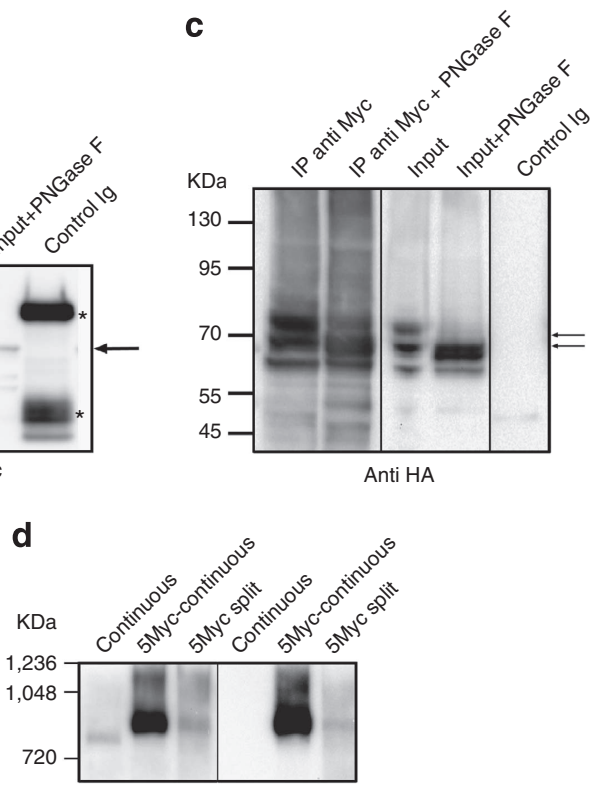

Figure 2 | Both $\mathrm{K}_{\mathrm{v}} \mathbf{1 0 . 1}$ demi-channels are detected in the same complex. (a) Biotinylation of surface proteins and subsequent pull down of labelled molecules allowed the detection of full-length channel using an anti-Kv10.1 $\mathrm{C}$-terminal antibody, and also of the C-terminal truncated protein when expressed either alone or as split channel. (b) Co-immunoprecipitation of $\mathrm{N}$ - and $\mathrm{C}$ - terminal demi-channels. The $\mathrm{N}$-terminal fragment was labelled with $5 \times \mathrm{Myc}$ and the $\mathrm{C}$-terminal was $4 \times \mathrm{HA}$ tagged. Immunoprecipitation with HA-tag pulled down a fragment of size compatible with the $\mathrm{N}$-terminal demi-channel (arrow), recognized by anti-Myc immunoblot. Asterisks indicate bands corresponding to the antibody used to immunoprecipitate. (c) Immunoprecipitation with anti-Myc also pulled HA-tagged fragments detected as a double band (arrows). The migration distance of the upper band was modified by deglycosylation (PNGase F lanes), as expected for the C-terminal fragment of $K_{V} 10.1$, which contains the glycosylated residues. Input lanes were loaded with the extract corresponding to half an oocyte; the equivalent to 30 oocytes were used to immunoprecipitate. (d) Native electrophoresis and immunoblot shows the presence of a complex with size similar to that of the continuous channel when demi-channels were expressed together, recognized by both anti-Myc and anti- $\mathrm{K}_{\mathrm{V}} 10.1$ antibodies.

properties typical of both voltage sensor and pore modules should be conserved in the split channel. A defining feature of $\mathrm{K}_{\mathrm{V}} 10.1$ is its high sensitivity to the prepulse potential ${ }^{30}$. The channel activates faster the more depolarized the potential before the stimulus (Fig. 3), and this phenomenon is strongly dependent on extracellular magnesium through interaction with residues located in the voltage-sensing module of the channel ${ }^{31}$. Moderate concentrations of $\mathrm{Mg}^{2+}$ slow down the activation and make the phenomenon most evident (Fig. 3a). The activation of the split channel was slower under all experimental conditions, but the dependence on the prepulse potential was preserved, as depicted in Fig. 3b. A comparison between the continuous and the split channel in the presence of $1 \mathrm{mM}$ extracellular $\mathrm{Mg}^{2+}$ is shown in Fig. 3c. Over $1 \mathrm{mM}$, the activation of the split channel was so slow that the changes induced by hyperpolarized prepulses became less obvious (Fig. 3d). In summary, the split channel maintains both the dependence on the prepulse potential and dependence on extracellular $\mathrm{Mg}^{2+}$

To further confirm that the activation of the split channel still depends on the voltage-sensor domain, we co-expressed a mutated $\mathrm{N}$ terminus where the voltage dependence had been shifted by neutralization of one of the positive charges in S4 (R336Q) with the wild-type C-terminal module. The resulting split channel displayed a shift of voltage dependence with respect to the wild-type split (Fig. 4a) comparable to the one observed in the continuous channel, strongly indicating that the voltage dependence observed in the split channel is indeed conferred by the properties of the voltage-sensing module.

To probe the properties of the permeation module of the split channel, we used astemizole, which is a relatively well-studied blocker of channels of the KCNH family ${ }^{32}$. The crucial residues for astemizole block lie in the C-terminal half of the channel (F468) (ref. 33), and we expected them to be largely conserved in the split channel. Indeed, astemizole still blocked both the split and the channel without a S4-S5 linker, although the $\mathrm{IC}_{50}$ was shifted to the right in both cases (Fig. 4b). In addition, we generated a split channel carrying a mutation in the deep pore of $\mathrm{K}_{\mathrm{V}} 10.1$ that abolishes permeation $(\mathrm{G} 440 \mathrm{~S})^{34}$. This combination did not give rise to detectable currents in oocytes (Fig. 4c-e), further indicating that the currents detected in the presence of the split are not due to endogenous overproduced oocyte channels. Fig. $4 \mathrm{e}$ shows the average current-voltage relationships obtained from 10 oocytes in those experiments.

Altogether, the expression of $\mathrm{K}_{\mathrm{V}} 10.1$ as two independent proteins containing the voltage-sensing and the permeability module, respectively, generated currents recapitulating properties attributable to either module, strongly indicating that the detected current depends on the association and interaction of both independent proteins, that correctly assemble and generate functional channels in the absence of physical continuity between them.

Functional expression of other S4-S5 split KCNH channels. A relevant question is whether our observations respond to a peculiar behaviour of $\mathrm{K}_{\mathrm{V}} 10.1$ or are rather extendable to other channels. We therefore tested two other KCNH members, $\mathrm{K}_{\mathrm{V}} 10.2$ (Eag2, encoded by KCNH5) and $\mathrm{K}_{\mathrm{V}} 11.1$ (HERG, encoded by KCNH2). $\mathrm{K}_{\mathrm{V}} 10.2$ shares high homology with $\mathrm{K}_{\mathrm{V}} 10.1$, and was truncated at $\mathrm{Y} 344$, the position equivalent to $\mathrm{Y} 347$ of $\mathrm{K}_{\mathrm{V}} 10.1$. Expression of $\mathrm{K}_{\mathrm{V}} 10.2$ wild type (Fig. $5 \mathrm{a}$ ) is in our hands much less efficient than that of $K_{V} 10.1$, and so was also the case for the split channel (Fig. 5b). The conductance/voltage plot of the $\mathrm{K}_{\mathrm{V}} 10.2$ split channel showed a marked shift to more positive potentials and thereby lost the very negative activation threshold of the parental channel (Fig. 5c). Nevertheless, we could observe potassium currents compatible with $\mathrm{K}_{\mathrm{V}} 10.2$ in oocytes injected with the two truncated proteins.

$\mathrm{K}_{\mathrm{V}} 11.1$ (HERG) could also be expressed as combination of two demi-channels: an initial half truncated after Y545, in the middle of the putative S4-S5 linker, thus corresponding to residues 1-545, and a truncated channel covering residues 546-1159. Voltage-evoked currents reminiscent of those of continuous $\mathrm{K}_{\mathrm{V}} 11.1$ (Fig. 6a) were obtained upon expression of the split HERG. Thus, while the magnitude of the wild-type peak tail current measured at $-50 \mathrm{mV}$ in $2 \mathrm{mM}$ extracellular $\mathrm{K}^{+}$ amounted $1.68 \pm 0.32 \mu \mathrm{A}$ (mean \pm s.e., $n=26, N=11$ ), the Y545 split peak tail current reached $0.36 \pm 0.06 \mu \mathrm{A}$ (mean \pm s.e., $n=19$, $N=9$ ). This value increased to $0.6 \pm 0.1 \mu \mathrm{A}$ (mean \pm s.e.) when the tail current magnitude was estimated by extrapolation at zero 
a
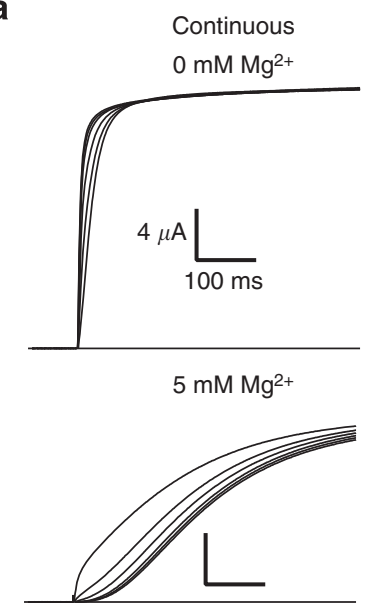

b

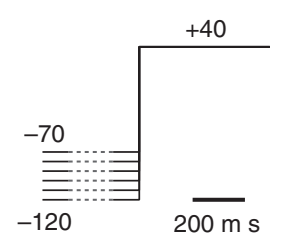

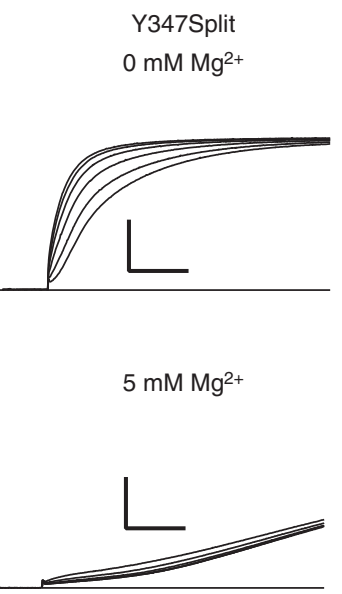
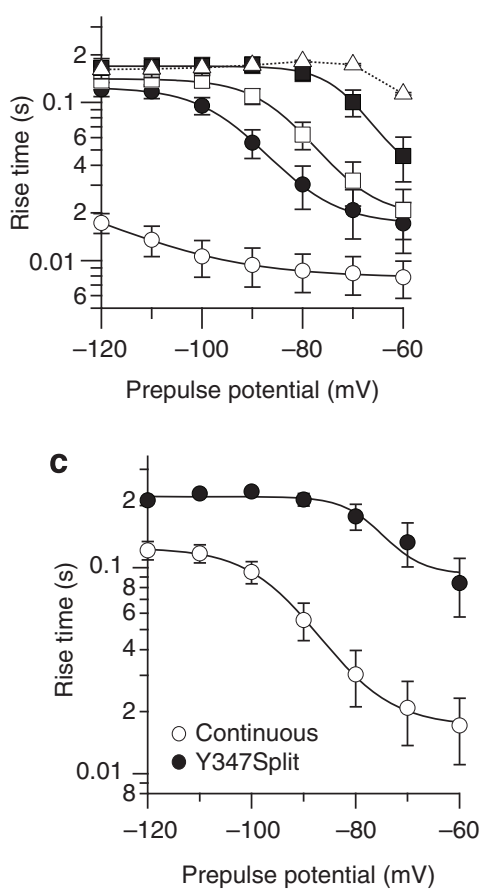

$\left[\mathrm{Mg}^{2+}\right]$

- $0 \mathrm{mM}$

- $1 \mathrm{mM}$

$\square 2 \mathrm{mM}$

- $5 \mathrm{mM}$

... $\triangle \cdots 10 \mathrm{mM}$

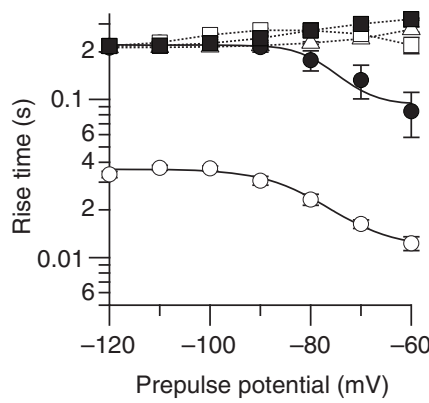

d

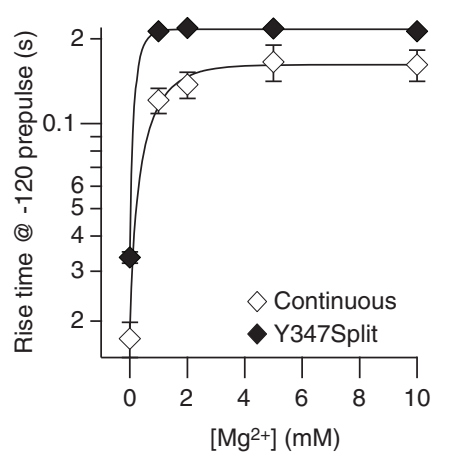

Figure 3 | Split channels retain dependence on the prepulse potential and on extracellular $\mathbf{M g}^{\mathbf{2}+}$. (a,b) Representative current traces obtained following the depicted protocol, holding the oocyte for $5 \mathrm{~s}$ (indicated in the schematic as a dotted line) at the indicated potential and immediately stimulating to $+40 \mathrm{mV}$ in the absence (upper traces) or the presence (middle traces) of $5 \mathrm{mM}$ extracellular $\mathrm{Mg}^{2+}$. Both wild-type (a) and split (b) channels show accelerated activation at less hyperpolarized potentials. Also in both cases, $\mathrm{Mg}^{2}+$ slows down the activation. The effect of prepulse potential at different $\mathrm{Mg}^{2+}$ concentrations is depicted in the lowest panels $(n=5-8)$. Over $1 \mathrm{mM}$, the activation of the split channel is so slow that the dependence on prepulse is not any longer evident. The solid lines indicate fits to sigmoid functions, dotted lines represent polynomial fits. (c) Comparison of data (from $\mathbf{a}, \mathbf{b}$ ) of continuous and split channel in $1 \mathrm{mM} \mathrm{Mg}^{2+}$. (d) Effect of $\mathrm{Mg}^{2+}$ concentration on the speed of activation of continuous and split channels. The activation was slower in the split channels (closed symbols) than in control (open symbols) under all conditions tested. Error bars represent s.e.

repolarization time to prevent the reduction in the peak current imposed by the very fast deactivation decay of the split tails (see below). As expected, no detectable currents were observed when any of the demi-channels were separately expressed in the oocytes (Supplementary Fig. 1). The split channel currents exhibited the typical $\mathrm{K}_{\mathrm{V}} 11.1$ inward rectification at positive voltages. The voltage dependence of the steady-state activation was only slightly shifted to positive potentials (Fig. 6b). Also, the voltagedependent activation rate of the split was only slightly slower than wild type at positive voltages (Fig. 6c). Alterations in the S4-S5 loop of $\mathrm{K}_{\mathrm{V}} 11.1$ are expected to produce an acceleration of deactivation ${ }^{35}$. Consistently, the deactivation time constant of the discontinuous channel was accelerated by an order of magnitude
(Fig. 7a). Finally, the inactivation of the split $\mathrm{K}_{\mathrm{V}} 11.1$ was also similar to the wild-type channel (Fig. 7b) and appeared clearly slowed when the extracellular $\mathrm{K}^{+}$level was raised from 2 to $50 \mathrm{mM}$ (refs 27,36). Thus, co-expression of the two demichannels rendered currents with the predicted properties, further indicating that the S4-S5 linker is indeed interrupted, and that there is no covalent fusion of the two proteins during synthesis, assembly and/or trafficking.

Similar to the results observed with the split $\mathrm{K}_{\mathrm{V}} 10.1$ channel, the currents elicited after co-expressing the two truncated HERG halves were sensitive to E-4031 (Fig. 8a), a very specific blocker of $\mathrm{HERG}^{37}$, although with lower affinity than the intact channel ${ }^{38}$ Furthermore, a permeation-inhibiting mutation $(\mathrm{G} 628 \mathrm{~S})^{39,40^{\circ}}$ 

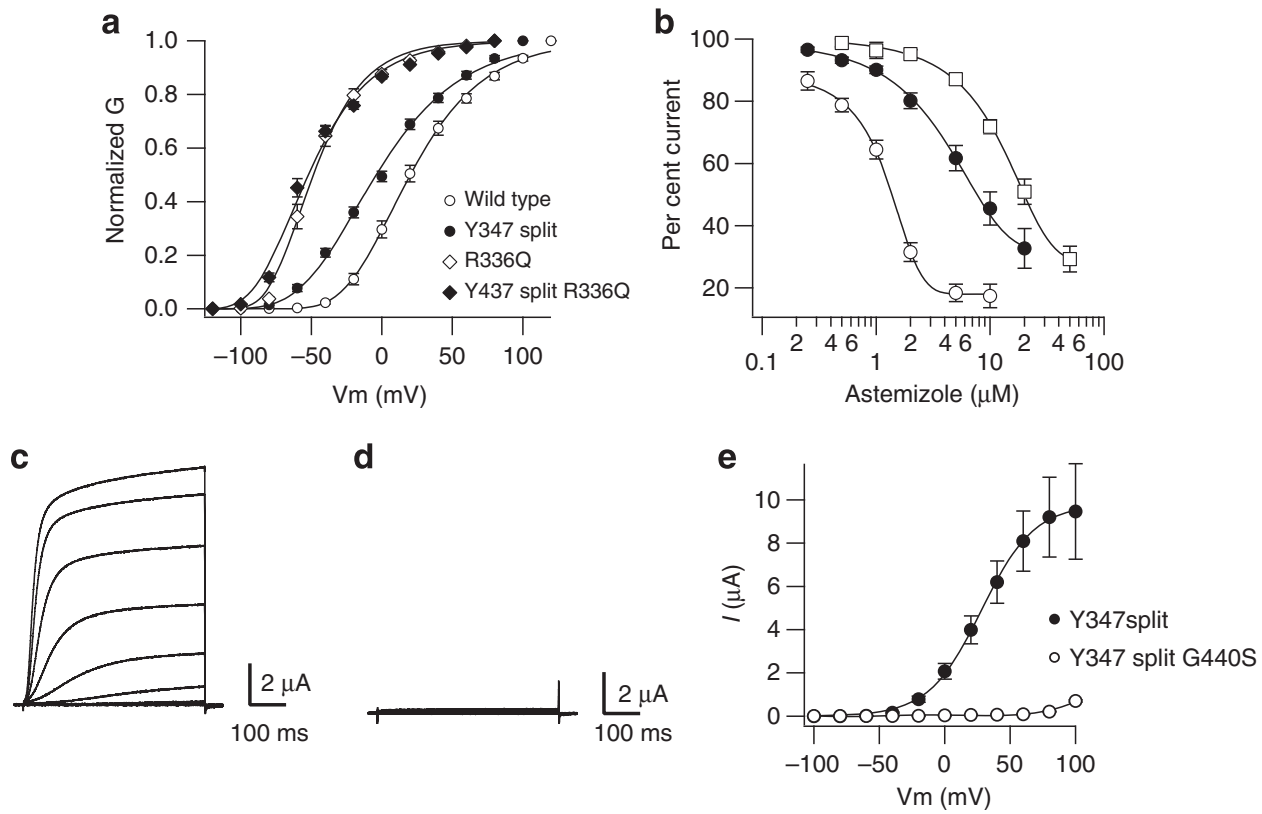

Figure 4 | Split channels retain properties residing in both the voltage-sensing and permeation modules. (a) Mutation of the voltage-sensor domain has similar effects in continuous and split channels. Neutralization of a positive charge in the S4 domain (R336Q) shifts the activation potential to hyperpolarized values regardless of the integrity of the S4-S5 linker (Open circles, wild type; closed circles, split channel; open diamonds, R336Q; closed diamonds, split R336Q; $n=7$; error bars, s.e.). (b) Blockade by astemizole, whose structural determinants lie in the C-terminal part of the protein, is conserved both in channels with interrupted S4-S5 (closed circles, $n=6-7$ ) or lacking completely the linker (squares, $n=2-4$ ), albeit with reduced affinity as compared with control (open circles, $n=9-16)$. (c-e) A pore mutation precluding $\mathrm{K}^{+}$permeation abolishes the activity also of split channels (c, wild-type split; d, G440S mutant split) (e) I/V relationship of wild-type and mutant split channels constructed with values obtained from 10 oocytes. Error bars represent s.e.

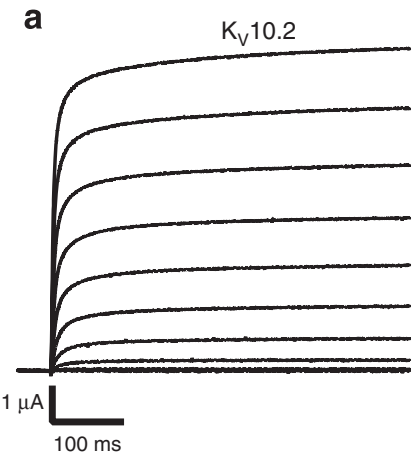

b

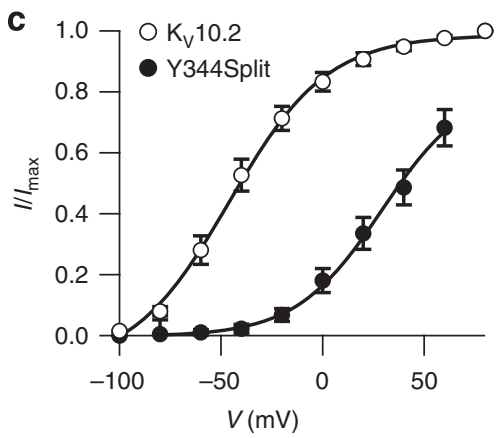

Figure 5 | Interruption of the S4-S5 linker renders also functional $K_{v} \mathbf{1 0 . 2}$ channels. Current amplitudes were always bigger in continuous (a) than split (b) channels. The split channel (solid symbols, $n=4$ for this plot) also required larger depolarization than the continuous channel (open symbols, $n=14$ ) to be activated (c). Error bars represent s.e. equivalent to the one reported for $\mathrm{K}_{\mathrm{V}} 10.1$ in Fig. 4 also abolished functional expression of the split HERG channel (Fig. 8b). Introduction in the split C-terminal permeation module of a S620T pore domain point mutation, known to antagonize $\mathrm{K}_{\mathrm{V}} 11.1$ inactivation $^{27}$, reduced (at $2 \mathrm{mM} \mathrm{K}^{+}$) or eventually abolished (in $50 \mathrm{mM}$ ) inactivation of the split channel (Fig. 8c), without affecting the voltage dependence of activation (Fig. 8c) or deactivation (Supplementary Fig. 2). Finally, deletion of the $\mathrm{N}$ terminus proximal domain (residues 138-373; see ref. 41), which shifts the activation voltage dependence towards more negative values and accelerates activation of HERG channels, induced similar effects when performed in the split N-terminal half of the channel (Fig. 9 and refs 35,41). In conclusion, the voltage sensor of the initial half is able to confer near normal voltage-dependent properties to the assembled construct, and the functional properties of the pore domain leading to the characteristic voltage-dependent inactivation of HERG are maintained in the co-assembled channels. Altogether our data unequivocally demonstrate that the permeation properties of the split channels are determined by the C-terminal domain, but it is the initial voltage sensor-containing module what crucially determines the voltage-dependent properties of the assembled protein.

Hybrid split channels from different KCNH family members. To check for the specificity of the split channel assembly, we tried combinations of the $\mathrm{N}$ - and C-terminal halves from $\mathrm{K}_{\mathrm{V}} 10.1$ and $\mathrm{K}_{\mathrm{V}} 11.1$ as well as $\mathrm{K}_{\mathrm{V}} 10.1$ and $\mathrm{K}_{\mathrm{V}} 10.2$. No active channels were recorded when the voltage sensor-containing $\mathrm{N}$-terminal half of $\mathrm{K}_{\mathrm{V}} 11.1$ was co-expressed with the C-terminal half of $\mathrm{K}_{\mathrm{V}} 10.1$. However, co-injection in the oocytes of the $\mathrm{N}$-terminal voltagesensing module of $\mathrm{K}_{\mathrm{V}} 10.1$ (corresponding to residues 1-347, this last one in the middle of the S4-S5 linker) with the C-terminal 

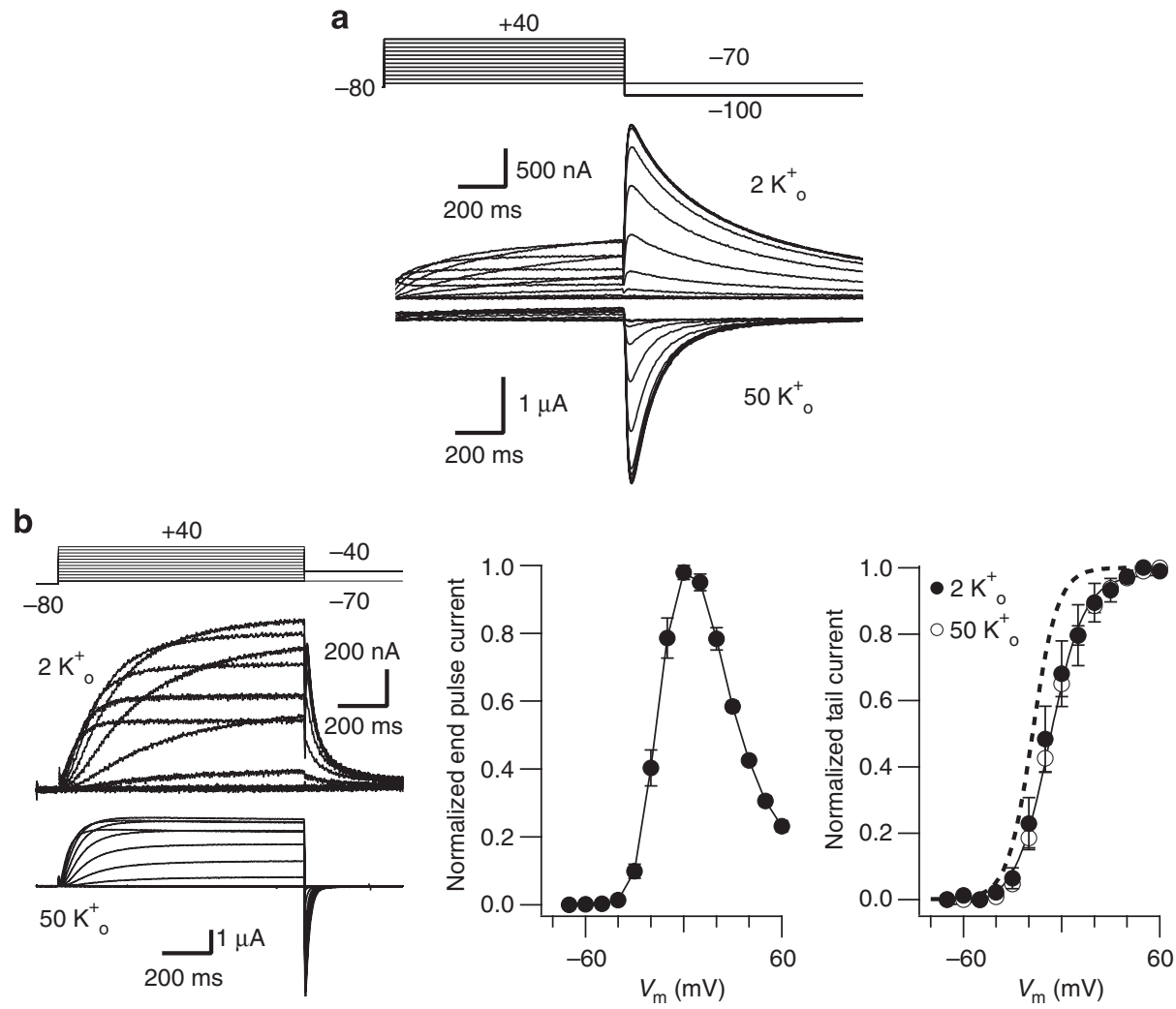

C
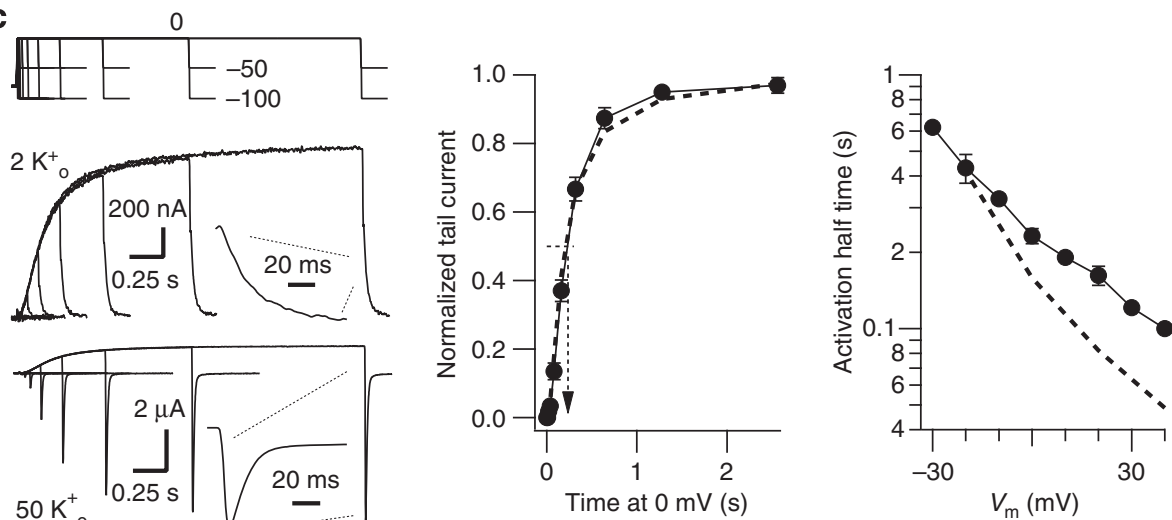

Figure 6 | Functional expression and activation kinetics of continuous and Y545 Split $\mathbf{K}_{\mathbf{v}} \mathbf{1 1 . 1}$ (HERG) channels. (a) Representative current traces of continuous wild-type HERG channels in response to the protocol shown at the top. Extracellular medium containing 2 and $50 \mathrm{mM} \mathrm{KCl}$ was used as indicated. (b) Voltage-dependent activation of $\mathrm{Y} 545 \mathrm{Split} \mathrm{K}_{\mathrm{V}} 11.1$ channels. Left, raw current traces recorded in 2 and $50 \mathrm{mM}$ extracellular $\mathrm{K}^{+}$in response to $1 \mathrm{~s}$ depolarization pulses between -70 and $+60 \mathrm{mV}$ at $10 \mathrm{mV}$ intervals from a holding potential of $-80 \mathrm{mV}$, followed by a repolarizing step to -40 $\left(2 \mathrm{mM} \mathrm{K}^{+}\right)$and $-70\left(50 \mathrm{mMK}^{+}\right) \mathrm{mV}$. Centre, averaged / versus $V$ relationship measured at the end of the depolarization step in $2 \mathrm{mM}$ external $\mathrm{K}^{+}$ $(n=8)$. Note the typical $n$-shaped curve due to the strong rectification as a result of the typical $K_{v} 11.1$ slow activation and fast inactivation overlap at positive voltages. Right, plots of normalized peak tail currents as a function of depolarizing voltage in 2 (closed circles) and $50 \mathrm{mM}$ (open circles) extracellular $\mathrm{K}^{+}(n=13)$. A curve from wild-type continuous $\mathrm{K}_{\mathrm{V}} 11.1$ channels obtained in the same conditions is shown as a dashed line for comparison. (c) Y545 Split $K_{V} 11.1$ voltage-dependent activation rates. Left, time course of current activation at $0 \mathrm{mV}$ in 2 (top traces) and $50 \mathrm{mM}$ (lower traces) extracellular $\mathrm{K}^{+}$. The duration of a depolarizing prepulse from a holding potential of $-80 \mathrm{mV}$ was varied and followed by a repolarization step to -50 and $-100 \mathrm{mV}$ in 2 and $50 \mathrm{mM}$ external $\mathrm{K}^{+}$, respectively. An enhanced view of the tail currents at the end of the 2,560 ms depolarizing steps is shown in the insets. Centre, plot of normalized tail current magnitude versus depolarization time at $0 \mathrm{mV}(n=4)$. The plot was used to measure time necessary to attain half-maximum current magnitude (dashed arrow). Note the sigmoidal nature of the early activation time course during the initial tens of ms. Right, dependence of activation rates on depolarisation membrane potential $(n \geq 4)$. Values from wild-type non-split channels are shown as a dashed line for comparison. Error bars represent s.e.

permeation module of HERG (from residues 546 in the S4-S5 loop to 1,159 at the $\mathrm{C}$ terminus) yielded voltage-dependent currents that basically recapitulated those obtained with the $\mathrm{K}_{\mathrm{V}} 10.1$ split (Fig. 10a). No HERG-type inward rectification at positive voltages was observed with this combination, and only a little nonlinearity of the $\mathrm{I} / \mathrm{V}$ relationship at voltages positive to $+40 \mathrm{mV}$ was obtained in $2 \mathrm{mM}$ extracellular $\mathrm{K}^{+}$, that was virtually abolished by raising the concentration of the cation to $50 \mathrm{mM}$. Similarly, the voltage-sensing domain of $\mathrm{K}_{\mathrm{V}} 10.2$ was able to generate functional channels when combined with the pore 
a

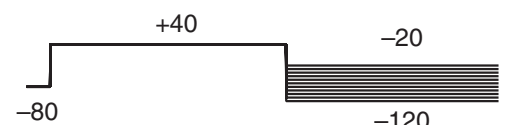

$-120$

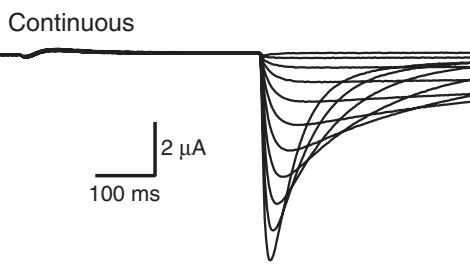

Y545 Split

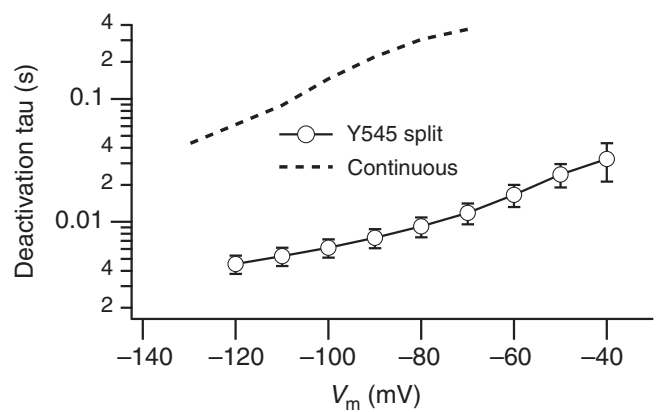

b
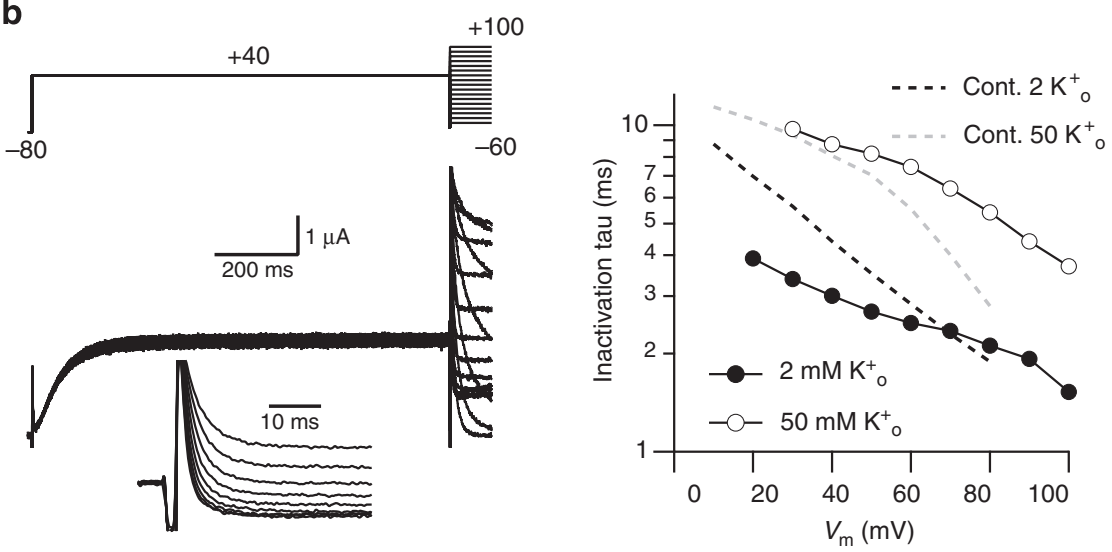

Figure 7 | Characterization of deactivation and inactivation properties of Y545 Split $\mathbf{K}_{\mathbf{v}} \mathbf{1 1 . 1}$ channels. (a) Voltage-dependent deactivation. Top panels, representative current traces recorded in $50 \mathrm{mM}$ extracellular $\mathrm{K}^{+}$during steps to potentials ranging from -20 to $-120 \mathrm{mV}$ at $10 \mathrm{mV}$ intervals, following depolarizing pulses to $+40 \mathrm{mV}$ from a holding potential of $-80 \mathrm{mV}$ as schematized at the top. Currents from oocytes expressing continuous wild-type (left) and Y545 Split $K_{v} 11.1$ (right) are shown for comparison. For the split, also an enhanced view of the peak tail currents during the repolarization steps is shown in the inset. Lower panel, plot of fast deactivation time constant for different repolarization voltages for split (open circles, $n=4$ ) and wild-type non-split channels (dashed line). Error bars, s.e. (b) Measurement of Y545 Split $K_{v} 11.1$ inactivation rates. Left panel, onset of fast inactivation at different voltages was studied in $2 \mathrm{mM}$ extracellular $\mathrm{K}^{+}$with the triple pulse protocol shown at the top, in which the channels were activated and inactivated with a $1 \mathrm{~s}$ prepulse to $+40 \mathrm{mV}$, followed by a second short prepulse to $-100 \mathrm{mV}$ to recover the channels from inactivation and a test pulse to different voltages from -60 to $+100 \mathrm{mV}$ at $10 \mathrm{mV}$ intervals to reinactivate them. Membrane currents starting at the end of the depolarizing prepulse for depolarizations between +20 and $+100 \mathrm{mV}$ at which the inactivation and deactivation kinetics barely overlap, are shown in the inset. Right panel, plot of time constants for the onset of inactivation in $2 \mathrm{mM}$ (closed circles) and $50 \mathrm{mM}$ (open circles) extracellular $\mathrm{K}^{+}$as a function of voltage, obtained from single-exponential fits to the decaying portion of the currents during the test pulses. Values from wild-type non-split channels at the same $\mathrm{K}^{+}$ concentrations are shown as black $(2 \mathrm{mM})$ and grey $(50 \mathrm{mM})$ dashed lines for comparison.

module of $\mathrm{K}_{\mathrm{V}} 10.1$ (Fig. 10b). Furthermore, the speed of activation of the $\mathrm{K}_{\mathrm{V}} 10.1 / \mathrm{K}_{\mathrm{V}} 11.1$ combination during the depolarization pulses became faster when the holding potential was maintained at more depolarized values (Fig. 10c), a typical signature of $\mathrm{K}_{\mathrm{V}} 10.1$ (Fig. 3) and $\mathrm{K}_{\mathrm{V}} 10.2$ channels $^{30}$ that is not exhibited by $\mathrm{K}_{\mathrm{V}} 11.1$ (ref. 41 ). This demonstrates not only that the ability to voltage-dependent gate the HERG pore is maintained upon heterologous co-assembly with the $\mathrm{K}_{\mathrm{V}} 10.1$ voltage sensor in the absence of physical continuity between them, but also that the voltage-dependent properties are mainly conferred by the voltage-sensor moiety of the split. Finally, the truncation of channels of the KCNA $\left(\mathrm{K}_{\mathrm{V}} 1.4\right)$ or KCNQ $\left(\mathrm{K}_{\mathrm{V}} 7.2 / 3\right)$ families did not produce functionally active channels. As discussed below, the reason(s) for this differential behaviour remains to be established.

\section{Discussion}

Our data demonstrate for the first time that the covalent link between voltage sensor and pore module is not necessary to confer voltage-dependent gating properties to $\mathrm{KCNH}$ channels. Our results challenge the classical view of voltage-dependent channel activation in which the outward movement of the S4 

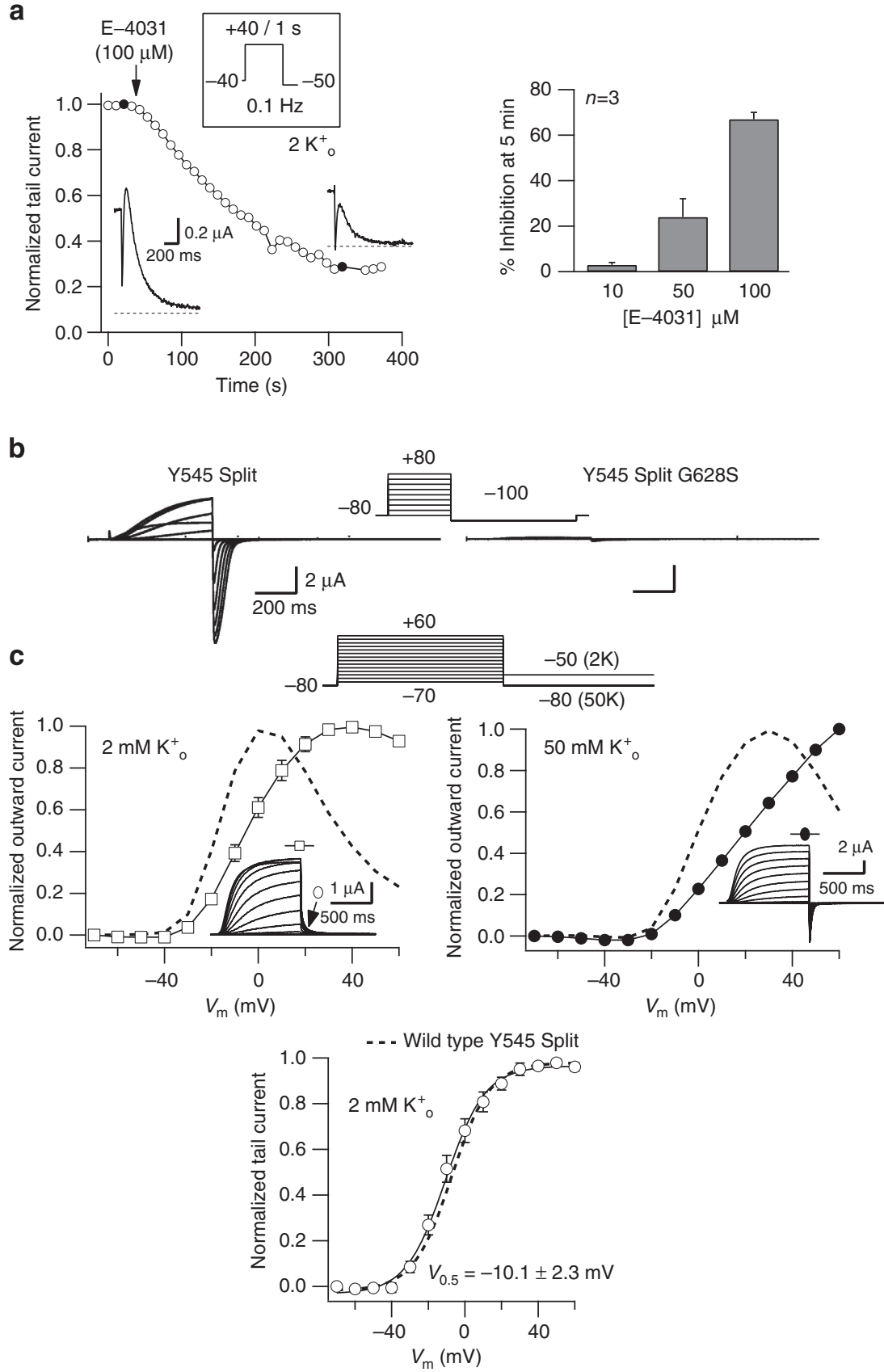

Figure 8 | Y545 Split HERG channels retain properties residing in the permeation module. (a) Inhibition of split HERG currents by E-4031.

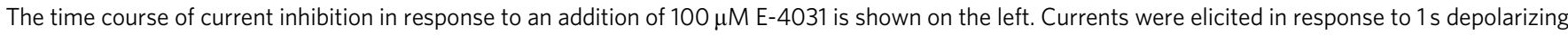
pulses to $+40 \mathrm{mV}$ from a holding potential of $-40 \mathrm{mV}$ followed by a repolarization step to $-50 \mathrm{mV}$. Pulses were delivered at a frequency of $0.1 \mathrm{~Hz}$. Representative tail current traces before and after 5 min of treatment with $\mathrm{E}-40131$ are shown on the insets. A bar histogram relating the extent of current inhibition with the concentration of the inhibitor is shown on the right. (b). Absence of functional expression upon co-injection of cRNAs encoding $\mathrm{N}$-terminal 1-545 and non-conducting C-terminal 546-1,159 $\mathrm{Kv} 11.1$ demi-channels carrying a G628S point mutation in the pore. Currents were obtained in $50 \mathrm{mM}$ extracellular $\mathrm{K}^{+}$using the voltage protocol shown at the top. Families of currents from non-mutant (left) and G628S (right) splits are shown. (c) Characterization of voltage-dependent currents elicited by co-expression of Kv11.1 demi-channels carrying a S620T point mutation in the pore domain. The voltage protocol used to elicit the currents is shown at the top. Representative families of currents recorded in response to the protocol are shown in the insets. Top panels, I versus $V$ relationships from normalized currents measured in $2 \mathrm{mM}$ (open squares, left) and $50 \mathrm{mM}$ (closed circles, right) extracellular $\mathrm{K}^{+}$at the end of the depolarization step. Lower panel, current/voltage relationship generated using peak tail currents measured in $2 \mathrm{mM}$ extracellular $\mathrm{K}^{+}$(open circles). Data from normally inactivating non-mutated split channels obtained in the same conditions are shown as dashed lines (Y545 Split) for comparison. Error bars represent s.e.

voltage-sensor helix tracks the $\mathrm{S} 4-\mathrm{S} 5$ linker that acting as a rigid mechanical lever pulls apart the N-terminal portion of S5 and the C-terminal end of S6 lining the channel gate to open it. Such a mechanism would be incompatible with voltage dependence in a split $\mathrm{KCNH}$ channel. What molecular system could be involved in coupling the voltage sensor to the pore module in the presence of a structurally interrupted S4-S5 linker? One possibility is that the C-terminal end of S4 and/or the initial half of the S4-S5 linker 

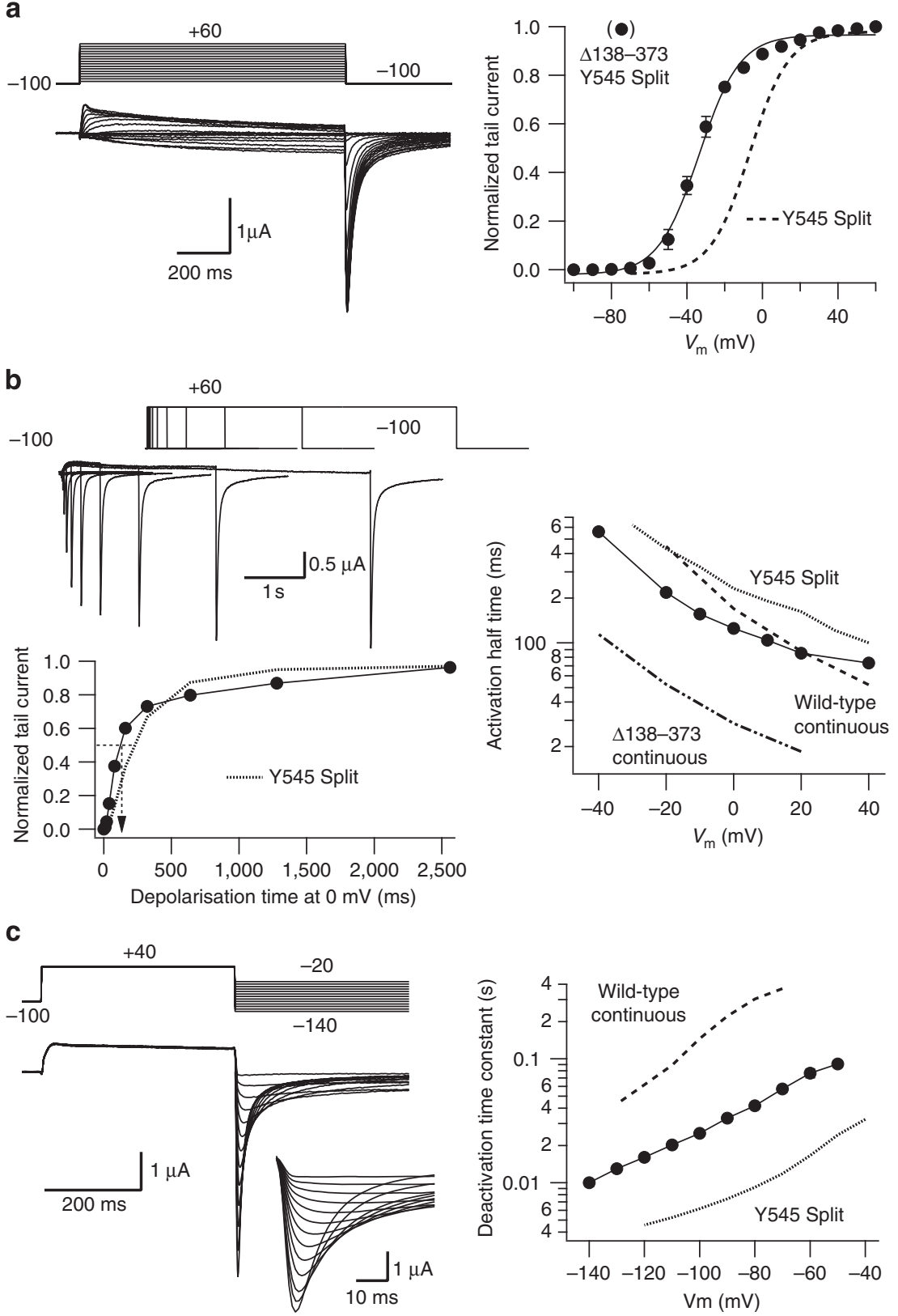

\section{Figure 9 | Characterization of voltage-dependent currents elicited by co-expression of $K_{\mathbf{v}} \mathbf{1 1 . 1}$ demi-channels carrying a $\Delta 138-373$ deletion.}

(a) Voltage-dependent activation of $\mathrm{K}_{\mathrm{V}} 11.1 \mathrm{C}$-terminal and $\mathrm{N}$-terminal demi-channels carrying a $\Delta 138-373$ deletion corresponding to the proximal domain located at the cytoplasmic segment, preceding helix S1 in the initial half of the voltage sensor module. Left panel, raw current traces recorded in $50 \mathrm{mM}$ extracellular $\mathrm{K}^{+}$in response to the voltage protocol shown at the top. Right panel, plot of normalized peak tail currents as a function of depolarizing voltage. A curve from non-deleted split channels obtained in the same conditions is shown as a dotted line for comparison. Error bars represent s.e. (b) Determination of voltage-dependent activation rates. Left panels, time course of current activation at $0 \mathrm{mV}$ studied in $50 \mathrm{mM}$ extracellular $\mathrm{K}^{+}$. Representative currents recorded in response to the illustrated voltage protocol are shown at the top. A plot of normalized peak current value versus depolarization time at $0 \mathrm{mV}$ is shown at the bottom. The time necessary to attain half-maximum current magnitude is marked with a dashed arrow. Right panel, dependence of activation rates on depolarization membrane potential. Values from wild-type non-split channels (Wild type continuous), non-deleted split channels (Y545 Split) and non-split channels lacking the proximal domain at the amino terminus ( $\Delta 138-373$ continuous) are also shown for comparison. (c) Voltage-dependent deactivation. Left panel, representative current traces recorded in $50 \mathrm{mM}$ extracellular $\mathrm{K}^{+}$during steps to potentials ranging from -20 to $-140 \mathrm{mV}$ with the voltage protocol schematized at the top. An enhanced view of the peak tail currents during the repolarization steps is shown in the inset. Right panel, plot of fast deactivation time constant for different repolarization voltages. Values from wild-type non-split channels (wild-type continuous) and non-deleted split channels (Y545 Split) are also shown for comparison.

pushes the S5-S6 module to maintain the gate closed at rest, whereas its movement upon depolarization allows for passive relaxation of the pore module to its default conducting conformation $^{42}$. Unlike Shaker-like $\mathrm{K}_{\mathrm{V}}$ channels $^{43}$, an intrinsically more stable open state has been proposed for
$\mathrm{K}_{\mathrm{V}} 11.1$ (refs 4,10,35,44). However, our data indicate that when injected alone the C-terminal domain does not give rise to detectable potassium currents, even though it is expressed at the plasma membrane, as suggested by the dominant negative effect of the non-permeant demi-channel modules, and directly 
a

$+80$
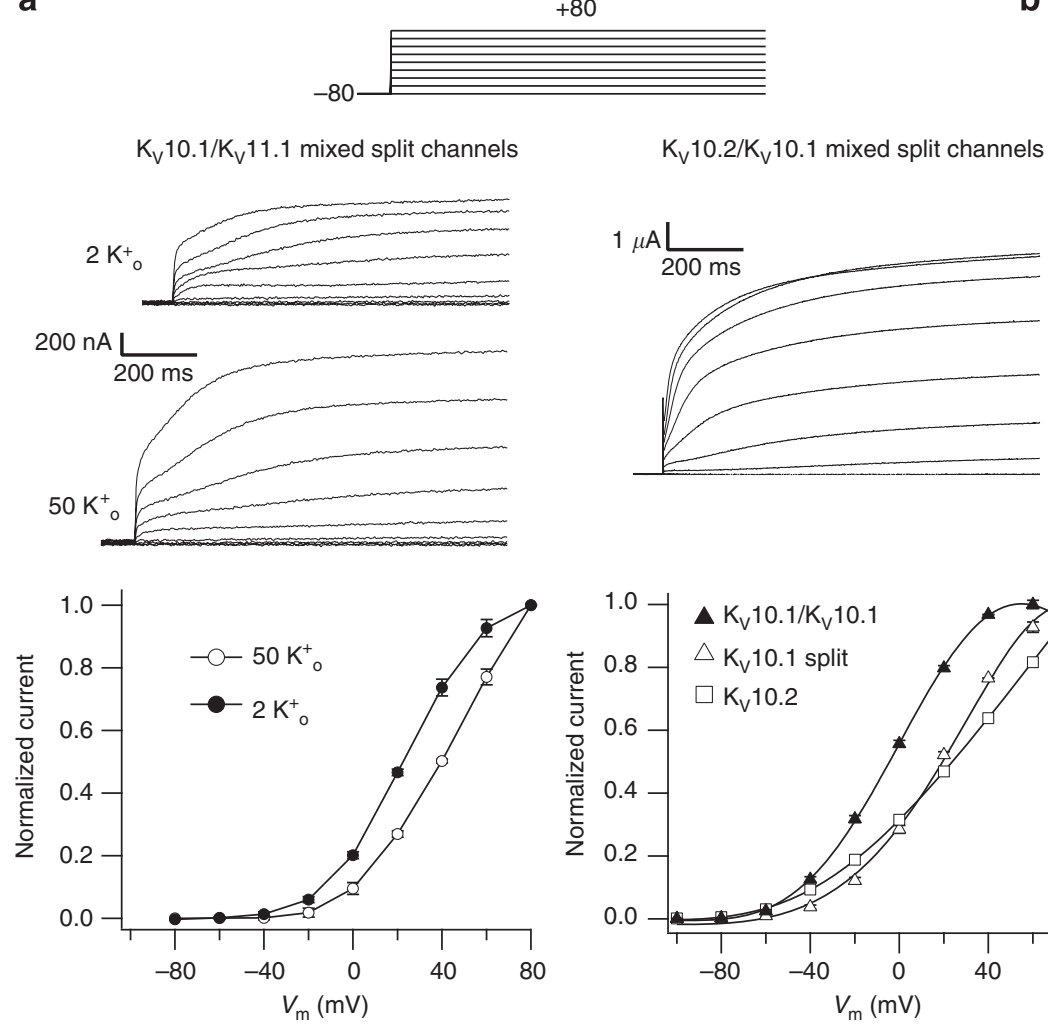

$\mathrm{K}_{\mathrm{V}} 10.2 / \mathrm{K}_{\mathrm{V}} 10.1$ mixed split channels

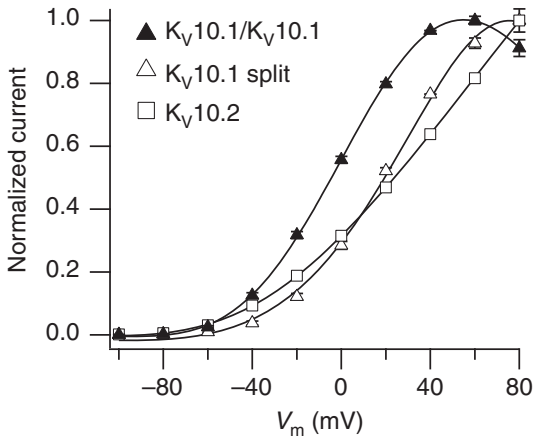

C

$+40$

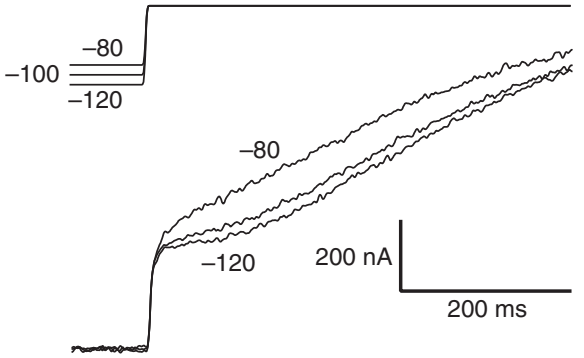

Figure 10 | Hybrid split channels yield voltage-dependent currents. (a) Voltage-dependent activation of the $\mathrm{K}_{v} 10.1 \mathrm{~N}$-terminal/Kv $11.1 \mathrm{C}$-terminal assembled demi-channels. Current traces as result of $500 \mathrm{~ms}$ depolarizations to potentials ranging between -80 and $+80 \mathrm{mV}$ in $20 \mathrm{mV}$ intervals in both 2 and $50 \mathrm{mM}$ extracellular $\mathrm{K}^{+}$are shown at the top. The normalized I/V relationships at both potassium concentrations are shown at the bottom.

(b) Voltage-dependent activation of the $\mathrm{K}_{\mathrm{v}} 10.2 \mathrm{~N}$-terminal/Kv10.1 C-terminal assembled demi-channels. Representative current records in $2 \mathrm{mM}$ extracellular $\mathrm{K}^{+}$are shown. Note the much higher current level obtained with this mixture as compared with a. Normalized $I / V$ relationships of the mixed demi-channels $(n=18)$ as compared with $\mathrm{K}_{\mathrm{V}} 10.1$ demi-channels $(n=23)$ and $\mathrm{K}_{\mathrm{V}} 10.2$ continuous channels $(n=17)$ are shown at the bottom. Error bars represent s.e. (c). Acceleration of current activation ('Cole-Moore' shift) by holding the $\mathrm{K}_{\mathrm{v}} 10.1 \mathrm{~N}$-terminal/Kv $11.1 \mathrm{C}$-terminal mixed demi-channels at more depolarized potentials. The traces correspond to representative currents obtained from oocytes held at the indicated holding potentials upon stimulation to $+40 \mathrm{mV}$ using the protocol represented at the top.

demonstrated by the biotinylation experiments in the case of $\mathrm{K}_{\mathrm{V}}$ 10.1. Further experiments would be necessary to know if this absence of permeability with the isolated pore module is due to a closed conformation of the pore of the demi-channel, a collapse of the pore loops, aggregation or misfolding of the protein in the absence of the other parts of the channel.

A second alternative to explain the functional integrity of the splits is that the end of S4 and/or the beginning of the S4-S5 loop are used to transmit a conformational non-covalent coupling of the voltage sensor movement to opening and closing of the gate at the bundle crossing of S6 (ref. 45). In this case, the interaction between these two structures would transfer the energy generated by $\$ 4$ movement, to push or pull the opening of the S6-limited gate $^{15}$. Indeed, previous work both in $\mathrm{K}_{\mathrm{V}} 11.1$ (ref. 10) and other
Shaker-like $\mathrm{K}_{\mathrm{V}}$ channels ${ }^{11,12,46}$ identified a direct interaction between the S4-S5 linker and the C-terminal portion of the S6 helix as a crucial component of the gating process. It has been proposed that this intrasubunit interaction is further complemented by a intersubunit interaction between the lower S4 and the S5 of the neighbouring subunit to drive the final cooperative gating transition leading to pore opening ${ }^{47-49}$. In this context, our results identifying the C-terminal end of S4 and/or the initial segment of the S4-S5 loop (but not the integrity of this linker) as a crucial component of the gating mechanism, point to the possibility that these type of interactions are also involved in voltage-dependent gating of the $\mathrm{KCNH}$ channels. Interestingly, a nearly unaltered functionality in channels carrying a cleaved backbone between the voltage-sensing and the pore domains 
would be consistent with the recently recognized need of a flexible $\mathrm{N}$-terminal segment in the S4-S5 linker for proper $\mathrm{K}_{\mathrm{V}} 11.1$ channel gating ${ }^{19,20}$. Altogether, this indicates that instead of a classical electromechanical coupling based in an S4-S5 loop acting as a rigid mechanical lever, at least for $\mathrm{KCNH}$ channels the coupling of the voltage-sensing and pore modules is based in an 'electrointeractional' mechanism probably involving the end of S4 and/or the beginning of the S4-S5 loop as well as the C-terminal portion of the S6.

The currents observed with the splits are very unlikely due to proteins other than the injected channels. Tagged $\mathrm{K}_{\mathrm{V}} 10.1$ demichannels are able to immunoprecipitate the counterpart in both directions, that is, precipitating the $\mathrm{N}$ terminus pulls down the C-terminal part and vice versa. Furthermore, the split channel is not only detectable on the surface of the oocyte, but it also shows glycosylation resembling the pattern obtained for the continuous channel. The C-terminal demi-channels are detected in native gels in a complex with a size compatible with association with the $\mathrm{N}$-terminal part. These results indicate that both demi-channels associate to form a functional potassium channel.

It is important to note that although maintenance structural integrity of the S4-S5 loop is not strictly necessary to confer voltage-dependent gating, our results also indicate the existence of subtle differences in the behaviour of the splits, that can be aggravated upon complete removal of the linker (see, for example, Fig. 1d). The overall current amplitude was reduced in all channels tested as compared with the respective continuous channel. In addition, splitting induces some alterations in voltage dependence and, at least at certain voltages, a slower activation rate both for $K_{V} 10.1$ and $K_{V} 11.1$ (Figs 3, 5). Also, the modifications in gating properties induced by removal of the $\mathrm{K}_{\mathrm{V}} 11.1 \mathrm{~N}$-terminal proximal domain, are less prominent in the split than in the continuous channel (Fig. 9 and refs 35,41). These observations suggest that the assembly of channels is less efficacious and, once assembled, the communication between the voltage sensing and the permeation modules is altered to a certain extent. On the other hand, some changes in deactivation were observed in split channels of both $\mathrm{K}_{\mathrm{V}} 10.1$ and 11.1. Thus, the marked acceleration of the $\mathrm{K}_{\mathrm{V}} 11.1$ split closing indicates that the S4-S5 segment is also relevant for deactivation, but that its exact role is probably determined by interactions with other parts of the protein, as repeatedly reported for this channel ${ }^{18,50-53}$. This can be expected from the recognized role of the S4-S5 linker as a coupler of voltage sensing to gate, but also as an integrator of cytoplasmic signals that modulate activation and deactivation gating 9 . Indeed, a direct interaction of some intracellular channel structures with the S4-S5 domain has been demonstrated previously (reviewed in ref. 5). In other words, our results indicate that although not completely necessary for voltagedependent gating, the S4-S5 segment still plays a relevant role for proper function also in $\mathrm{KCNH}$ channels. Previous studies with $\mathrm{K}_{\mathrm{V}} 11.1$ carrying mutations at residue 540 or after crosslinking this position to the end of helix S6 (refs 10,35) indicate that the $\mathrm{N}$-terminal end of the S4-S5 linker plays a crucial role in coupling voltage sensing to channel gating. A similar mechanism may be involved in voltage-dependent coupling of the splits. However, the rest of the S4-S5 linker, probably through interaction(s) with other parts of the channel (for example, the $\mathrm{N}$-terminal eag/PAS and/or proximal domains, or the C-linker immediately below the gate $9,17,19,20,41,50-53)$ can also strongly influence the properties and extent of such coupling. The reduced voltage sensitivity of split channels lacking a S4-S5 linker would reinforce this view.

Importantly, properties attributable to each part of the full channel, voltage-sensing domain and pore domain, are transferred to the corresponding split channels. Pore blockers such as astemizole and E-4031 inhibit currents through $\mathrm{K}_{\mathrm{V}} 10.1$ and HERG split channels, respectively; pore mutations in both the channels abolish permeation also in the split channels; mutation of the S4 segment induces shift in the same direction in both split and continuous channels and $\mathrm{K}_{\mathrm{V}} 10.1$ split channels retain a marked Cole-Moore shift. Furthermore, Cole-Moore shift, which is not detected in HERG channels, is conferred to $\mathrm{K}_{\mathrm{V}} 10.1 / \mathrm{K}_{\mathrm{V}} 11.1$ hybrid split channels by the voltage-sensing domain of $K_{V} 10.1$, reinforcing the concept of modularity of ion channels.

Up to now, the results obtained with the split KCNH channels were not reproduced with members of the KCNA or KCNQ families. This could be attributed to mechanistic differences in the process of gating, but also to differences in the biosynthesis of the different families. For example, KCNA channels depend on a $\mathrm{N}$-terminal $\mathrm{T}$ domain for tetramerization, while $\mathrm{KCNH}$ channels rely most primarily on C-terminal tetramerization domains, although there is indication that N-terminal domains affecting multimerization also exist ${ }^{54}$. Perhaps this results in inactive $\mathrm{N}$-terminal tetramers of KCNA channels, while the correct association of $\mathrm{KCNH}$ channels would be favoured by their multimerization domains. However, KCNQ channels also have C-terminal tetramerization domains ${ }^{55,56}$ as $\mathrm{KCNH}$, and we did not observe functional channels in this family either.

Finally, our data open new unanswered questions. Thus, the specific step(s) of the synthesis, processing, assembly, quality control or trafficking pathways at which the modules come together remain to be established. Also, the molecular determinants for specificity of recognition of the two channel halves are unknown, although our preliminary experiments suggest that at least the $\mathrm{N}$ terminus can be important to build fully functional $\mathrm{K}_{\mathrm{V}} 11.1$ channels. Further work will be necessary to provide adequate answers to these questions.

\section{Methods}

Molecular biology. The initial split channels were generated from $\mathrm{K}_{\mathrm{V}} 10.1$ or $\mathrm{K}_{\mathrm{V}} 10.2$ in the pSGEM oocyte expression vector (M. Hollmann, Bochum ${ }^{57}$ ) by inserting at the chosen position in a 'StopXStart' cassette, where $X$ is a particular restriction enzyme flanked by the TGA stop and ATG start codons. Sequences of oligonucleotides used are listed in Supplementary Table 1. In the case of the $\mathrm{N}$-terminal demi-channel, $X$ was a previously absent restriction enzyme. The fragment was then excised with $X$ and a second single cutter from the polylinker and ligated into the empty pSGEM. For the C-terminal demi-channels, $X$ was chosen among already present single cutters, now becoming a double cutter. Restriction with this enzyme and subsequent religation of the larger DNA fragment resulted in the deletion of the $\mathrm{N}$-terminal part of the sequence. $\mathrm{K}_{\mathrm{V}} 10.1$ was split by inserting a 'StopBamHIStart' or 'StopEag1Start' cassette after position Y347. For $\mathrm{K}_{\mathrm{V}} 10.2$, 'StopHindIIIStart' or 'StopEag1Start' cassettes were used after Y344. Subsequent split channels were generated by deleting the sequence after the start codon up to the desired position (C-terminal constructs) or the sequence between the desired position and the stop codon (N-terminal constructs).

The split channels for $\mathrm{K}_{\mathrm{V}} 11.1$ were generated as PCR fragments containing the desired coding sequences that were inserted into the pSP64A + vector as HindIII-BamHI fragments. The N-terminal fragment was synthesized using a sense oligonucleotide containing a HindIII site, a Kozak's signal and the sequences for the initial eight $K_{V} 11.1$ residues together with an antisense oligonucleotide carrying the coding sequence for residues 535 to 545, a stop codon and the BamHI-recognition site. For the C-terminal $K_{V} 11.1$ fragment synthesis, the sense oligonucleotide was designed to contain a HindIII site, a Kozak's sequence and the start codon followed by the 546 to $557 \mathrm{~K}_{\mathrm{V}} 11.1$ coding sequence, whereas the antisense oligonucleotide covered the last $K_{V} 11.1$ residues $(1,049-1,059)$ and carried a stop codon and the BamHI-recognition sequence. The S620T C-terminal fragment was generated the same way using a template carrying the mutation ${ }^{41}$. Sequences of oligonucleotides used are listed in Supplementary Table 1.

Deletions and point mutations were generated using the Quick Change Kit (Agilent Technologies).

Tagged fragments were generated by overlapping PCR. Sequences of oligonucleotides used are listed in Supplementary Table 1. The 5-Myc tag was obtained by PCR from a tagged $\mathrm{K}_{\mathrm{V}} 7.2$ construct (a generous gift from A. Villarroel, Biophysics Unit, UPV-EHU ${ }^{58}$ ) and overlapped to the $\mathrm{K}_{\mathrm{V}} 10.1 \mathrm{~N}$-terminal demichannel. The 4-HA tag was generated by insertion of a synthetic oligonucleotide encoding two HA tags into a fragment with two additional HA tags generated by PCR and overlapped to the C-terminal constructs.

All constructs were confirmed by sequencing. 
cRNA synthesis and oocyte microinjection. cRNAs from pSGEM and pSP64A + constructs were prepared in vitro from linearized templates using the T7 or SP6 promoters, respectively, and the mMessage mMachine kit (Ambion). Xenopus laevis oocytes were microinjected with a total amount of cRNA ranging from 5 to $25 \mathrm{ng}$ using similar quantities of split channels cRNAs in a final volume of $50 \mathrm{nl}$. Oocytes were kept at $18^{\circ} \mathrm{C}$ in $\mathrm{ND} 96(96 \mathrm{mM} \mathrm{NaCl}, 2 \mathrm{mM} \mathrm{KCl}, 0.2 \mathrm{mM}$ $\mathrm{CaCl}_{2}, 2 \mathrm{mM} \mathrm{MgCl}_{2}, 0.5 \mathrm{mM}$ theophylline, $5 \mathrm{mM}$ HEPES, $\mathrm{pH}$ 7.5) or OR-2 ( $82.5 \mathrm{mM} \mathrm{NaCl}, 2 \mathrm{mM} \mathrm{KCl}, 2 \mathrm{mM} \mathrm{CaCl}, 2 \mathrm{mM} \mathrm{MgCl}_{2}, 1 \mathrm{mM} \mathrm{Na}_{2} \mathrm{HPO}_{4}$, $10 \mathrm{mM}$ HEPES, at $\mathrm{pH}$ 7.5) solutions.

Surface biotinylation. We used the Surface Biotinylation kit (Pierce). Oocytes expressing the corresponding constructs were washed with PBS and incubated for $30 \mathrm{~min}$ at $4{ }^{\circ} \mathrm{C}$ with $1 \mathrm{mg} \mathrm{ml}^{-1}$ sulfo-NHS-biotin under continuous agitation. The reaction was stopped by addition of $25 \mu \mathrm{l}$ quencher. Oocytes were then homogenized by pipetting through 1,000 and $100 \mu \mathrm{l}$ tips in $20 \mu \mathrm{l}$ per oocyte $1 \%$ Triton-X$100,150 \mathrm{mM} \mathrm{NaCl}, 20 \mathrm{mM}$ Tris- $\mathrm{HCl}, 5 \mathrm{mM} \mathrm{MgCl}, 5 \mathrm{mM}$ EDTA and protease inhibitors (Complete; Roche). The homogenate was incubated $30 \mathrm{~min}$ on ice and centrifuged twice at $20,000 \mathrm{~g}$ for $3 \mathrm{~min}$. The pellet was discarded and the clarified extract was incubated with Neutravidin resin for $60 \mathrm{~min}$ at room temperature. The resin was washed three times and finally the bound proteins were eluted by incubation with NuPage LDS sample buffer for $60 \mathrm{~min}$ at room temperature. The eluted proteins were concentrated by precipitation with trichloroacetic acid, and then reconstituted in LDS sample buffer and separated by polyacrylamide gel electrophoresis.

Immunoprecipitation and immunobloting. Ninety-six hours after injection of cRNA coding for the N-terminal $\mathrm{K}_{\mathrm{V}} 10.1$ demi-channel labelled with $5 \times$ Myc plus the C-terminal $4 \times$ HA-tagged demi-channel, 30 oocytes per group were collected and lysed as above. The pellet was discarded and the supernatant was precleared $1 \mathrm{~h}$ at $4{ }^{\circ} \mathrm{C}$ with $25 \mu \mathrm{l}$ Protein G-Magnetic Beads (New England Biolabs). Immunoprecipitation was performed for $1 \mathrm{~h}$ at $4{ }^{\circ} \mathrm{C}$ on the precleared lysate with $3 \mu \mathrm{g}$ anti-c-Myc (Santa Cruz cat \# sc-40), $2 \mu \mathrm{g}$ anti-HA (Roche cat. \#11867423001) or $2 \mu \mathrm{g}$ control mouse IgG $2 \mathrm{~b}$ (Abcam) and 90 min with $50 \mu \mathrm{l}$ Protein G-Magnetic Beads. The beads were washed three times with $0.1 \%$ Triton-X-100, $50 \mathrm{mM}$ Tris$\mathrm{HCl}, 300 \mathrm{mM} \mathrm{NaCl}, 5 \mathrm{mM}$ EDTA plus protease inhibitors. Immunoprecipitated proteins were recovered by heating at $70{ }^{\circ} \mathrm{C}$ for $10 \mathrm{~min}$ in LDS sample buffer (Invitrogen). Samples to be deglycosylated with PNGase F (Sigma) were resuspended in $16.5 \mu \mathrm{l}$ water to be digested overnight at $37^{\circ} \mathrm{C}$. For immunoblot analysis, total protein was separated by SDS-polyacrylamide gel electrophoresis, probed with anti-HA (1:1,000, Roche cat. \#11867423001) or anti-c-Myc (1:1,000, Sigma cat \#M4439). The membranes were stripped and reprobed against the epitope used to immunoprecipitate. Secondary antibodies (HA conjugated) were used at 1: 10,000 dilution (mouse, GE Healthcare \#NA931V, rabbit, GE Healthcare \#NA934V and rat (Jackson Immunoresearch \#112-035-006). Crude extract from 1/2 oocyte was used as input control.

For Blue native electrophoresis, oocytes were washed and homogenized in $0.1 \mathrm{M}$ sodium phosphate buffer, $\mathrm{pH} 8.0$ containing: $0.4 \mathrm{mM}$ Pefabloc SC (Fluka, Buchs, Switzerland) plus 1.5\% digitonin (Fluka). Extracts were incubated $10 \mathrm{~min}$ on ice and cleared at $25,000 \mathrm{~g}$ for $10 \mathrm{~min} .5 \mu \mathrm{l}$ extract ( $1 / 2$ oocyte) were mixed with $20 \mu \mathrm{l}$ Native Sample Buffer plus 0.375\% G250 (NuPAGE) and run in $4-16 \%$ precast Native Gels (Invitrogen) at $150 \mathrm{~V}$ in dark blue buffer for $45 \mathrm{~min}$ and at $250 \mathrm{~V}$ in light blue buffer. Semi-dry blotting was performed on polyvinylidene difluoride membranes, and immunodetection was performed as above. The anti- $\mathrm{K}_{\mathrm{V}} 10.1$ antibody (polyclonal 9391 (ref. 59)) was used at a dilution of $1: 1,500$.

Uncropped images of blots are presented in Supplementary Fig. 3.

Electrophysiology. Two-electrode voltage-clamp recordings were performed at room temperature 2-5 days after injection, using Turbo TEC-10CD and -10C amplifiers (NPI electronics). The intracellular electrodes had resistances of 0.5-1.0 M $\Omega$ when filled with 2 or $3 \mathrm{M} \mathrm{KCl}$. For $\mathrm{K}_{\mathrm{V}} 10$ recordings, the extracellular measuring solution contained $115 \mathrm{mM} \mathrm{NaCl}, 2.5 \mathrm{mM} \mathrm{KCl}, 1.8 \mathrm{mM} \mathrm{CaCl}_{2}, 10 \mathrm{mM}$ HEPES/NaOH, pH 7.2, with or without the indicated concentrations of $\mathrm{MgCl}_{2}$. $\mathrm{K}_{\mathrm{V}} 11.1$ currents and those of the $\mathrm{K}_{\mathrm{V}} 10.1 / \mathrm{K}_{\mathrm{V}} 11.1$ mixed demi-channels were routinely recorded in OR-2 medium. For recordings in high extracellular $\mathrm{K}^{+}, \mathrm{KCl}$ was increased to 50 or $60 \mathrm{mM}$ substituting $\mathrm{NaCl}$. Oocytes showing membrane potentials more positive than $-30 \mathrm{mV}$ and holding currents bigger than $200 \mathrm{nA}$ at $-80 /-100 \mathrm{mV}$ after impalement with the first and second electrode, respectively, were discarded. Data acquisition and analysis were performed with the PulsePulseFit (HEKA Electronics) and IgorPro (WaveMetrics) software packages. Ionic currents sampled at $1 \mathrm{KHz}$ were elicited using the voltage protocols indicated in the graphs. A $P / n$ method was used for leak and capacitive current subtraction, except in the case of Cole-Moore protocols, where no subtraction was performed. For $\mathrm{K}_{\mathrm{V}} 11.1$, the voltage dependence of activation was assessed by standard tail current analysis using depolarization pulses of variable amplitude. For very rapidly deactivating constructs, fitting the relaxation of the tail currents and extrapolating the magnitude of the decaying current to the time the depolarizing pulse ended were used to determine the amount of current passing through channels opened on depolarization without influence of rapid inactivation. Tail current magnitudes normalized to maximum were fitted with a Boltzmann function to estimate the $V_{1 / 2}$ and equivalent gating charge $\left(z_{\mathrm{g}}\right)$ :

$$
I_{\text {tail }} / I_{\max }=1 /\left[1+\exp \left(\left(V_{1 / 2}-V\right) z_{\mathrm{g}} F / R T\right)\right],
$$

where $V$ is the test potential and $F, R$ and $T$ are the Faraday constant, gas constant and absolute temperature, respectively. The time course of voltage-dependent activation was studied using an indirect envelope-of-tail-currents protocol, varying the duration of depolarization prepulses, and following the magnitude of the tail currents on repolarization. The time necessary to reach a half-maximal tail current magnitude was used to compare the speed of activation of the different channels. The rates of deactivation were determined from negative-amplitude biexponential fits to the decaying phase of tail currents using a function of the form:

$$
y=A_{\mathrm{f}} \exp \left(-\operatorname{inv} \tau_{\mathrm{f}} \cdot x\right)+A_{\mathrm{s}} \exp \left(-\operatorname{inv} \tau_{\mathrm{s}} \cdot x\right)+C
$$

where $\tau_{\mathrm{f}}$ and $\tau_{\mathrm{s}}$ are the time constants of fast and slow components, $A_{\mathrm{f}}$ and $A_{\mathrm{s}}$ are the relative amplitudes of these components and $C$ is a constant. In this case, the first cursor of the fitting window was advanced to the end of the initial hook because of the recovery of inactivation. Onset of fast inactivation was studied after activation and inactivation of the currents with a prepulse to positive voltages, followed by a second short prepulse to around $-100 \mathrm{mV}$ used to recover the channels from inactivation, and a subsequent test pulse to different voltages to reinactivate the channels. Time constants for the onset of inactivation were obtained from current traces fitting a single-exponential function to the decaying portion of the currents during the test pulses.

\section{References}

1. Yellen, G. The voltage-gated potassium channels and their relatives. Nature 419, 35-42 (2002).

2. Swartz, K. J. Sensing voltage across lipid membranes. Nature 456, 891-897 (2008).

3. Bezanilla, F. How membrane proteins sense voltage. Nat. Rev. Mol. Cell Biol. 9, 323-332 (2008).

4. Vardanyan, V. \& Pongs, O. Coupling of voltage sensors to the channel pore: a comparative view. Front. Pharmacol. 3, 145 (2012).

5. Barros, F., Dominguez, P. \& de la Peña, P. Cytoplasmic domains and voltagedependent potassium channel gating. Front. Pharmacol. 3, 49 (2012).

6. Sachyani, D. et al. Structural basis of a Kv7.1 potassium channel gating module studies of the intracellular C-terminal domain in complex with calmodulin. Structure 22, 1582-1594 (2014).

7. Gutman, G. A. et al. International Union of Pharmacology. LIII. Nomenclature and molecular relationships of voltage-gated potassium channels. Pharmacol. Rev. 57, 473-508 (2005).

8. Ohlenschlager, O., Hojo, H., Ramachandran, R., Gorlach, M. \& Haris, P. I. Three-dimensional structure of the S4-S5 segment of the Shaker potassium channel. Biophys. J. 82, 2995-3002 (2002).

9. $\mathrm{Ng}, \mathrm{C}$. A. et al. The S4-S5 linker acts as a signal integrator for HERG $\mathrm{K}^{+}$ channel activation and deactivation gating. PLoS ONE 7, e31640 (2012).

10. Ferrer, T., Rupp, J., Piper, D. R. \& Tristani-Firouzi, M. The S4-S5 linker directly couples voltage sensor movement to the activation gate in the human ether-à-go-go-related gene (hERG) $\mathrm{K}^{+}$channel. J. Biol. Chem. 281, 12858-12864 (2006).

11. Lu, Z., Klem, A. M. \& Ramu, Y. Coupling between voltage sensors and activation gate in voltage-gated $\mathrm{K}^{+}$channels. J. Gen. Physiol. 120, 663-676 (2002).

12. Long, S. B., Campbell, E. B. \& Mackinnon, R. Crystal structure of a mammalian voltage-dependent Shaker family $\mathrm{K}^{+}$channel. Science 309, 897-903 (2005)

13. Labro, A. J. et al. Kv channel gating requires a compatible S4-S5 linker and bottom part of S6, constrained by non-interacting residues. J. Gen. Physiol. 132, 667-680 (2008).

14. Jensen, M. O. et al. Mechanism of voltage gating in potassium channels. Science 336, 229-233 (2012).

15. Blunck, R. \& Batulan, Z. Mechanism of electromechanical coupling in voltagegated potassium channels. Front. Pharmacol. 3, 166 (2012).

16. de la Peña, P. et al. Demonstration of physical proximity between the $\mathrm{N}$ terminus and the S4-S5 linker of the human ether-a-go-go-related gene (hERG) potassium channel. J. Biol. Chem. 286, 19065-19075 (2011).

17. Tristani-Firouzi, M., Chen, J. \& Sanguinetti, M. C. Interactions between the S4-S5 linker and the S6 transmembrane domain modulate gating of HERG $\mathrm{K}^{+}$ channels. J. Biol. Chem. 277, 18994-19000 (2002).

18. Sanguinetti, M. C. \& Xu, Q. P. Mutations of the S4-S5 linker alter activation properties of HERG potassium channels expressed in Xenopus oocytes. J. Physiol. 514, 667-675 (1999).

19. Hull, C. M., Sokolov, S., Van Slyke, A. C. \& Claydon, T. W. Regional flexibility in the S4-S5 linker regulates hERG channel closed-state stabilization. Pflugers Arch. 466, 1911-1919 (2014).

20. Van Slyke, A. C. et al. Mutations within the S4-S5 linker alter voltage sensor constraints in hERG $\mathrm{K}^{+}$channels. Biophys. J. 99, 2841-2852 (2010). 
21. del Camino, D., Holmgren, M., Liu, Y. \& Yellen, G. Blocker protection in the pore of a voltage-gated $\mathrm{K}^{+}$channel and its structural implications. Nature 403, 321-325 (2000).

22. Mitcheson, J. S., Chen, J. \& Sanguinetti, M. C. Trapping of a methanesulfonanilide by closure of the HERG potassium channel activation gate. J. Gen. Physiol. 115, 229-240 (2000).

23. Witchel, H. J. et al. The low-potency, voltage-dependent HERG blocker propafenone - molecular determinants and drug trapping. Mol. Pharmacol. 66, 1201-1212 (2004).

24. Wynia-Smith, S. L., Gillian-Daniel, A. L., Satyshur, K. A. \& Robertson, G. A. hERG gating microdomains defined by $\mathrm{S} 6$ mutagenesis and molecular modeling. J. Gen. Physiol. 132, 507-520 (2008).

25. Thouta, S. et al. Proline scan of the HERG channel S6 helix reveals the location of the intracellular pore gate. Biophys. J. 106, 1057-1069 (2014).

26. Terlau, H. et al. Extracellular $\mathrm{Mg}^{2}+$ regulates activation of rat eag potassium channel. Pflugers Arch. 432, 301-312 (1996).

27. Vandenberg, J. I. et al. hERG $\mathrm{K}^{+}$channels: structure, function, and clinical significance. Physiol. Rev. 92, 1393-1478 (2012).

28. Schmidt-Rose, T. \& Jentsch, T. J. Reconstitution of functional voltage-gated chloride channels from complementary fragments of CLC-1. J. Biol. Chem. 272, 20515-20521 (1997)

29. Napp, J., Monje, F., Stühmer, W. \& Pardo, L. A. Glycosylation of Eag1 (Kv10.1) potassium channels: intracellular trafficking and functional consequences. J. Biol. Chem. 280, 29506-29512 (2005).

30. Ludwig, J. et al. Functional expression of a rat homologue of the voltage gated ether á go-go potassium channel reveals differences in selectivity and activation kinetics between the Drosophila channel and its mammalian counterpart. EMBO J. 13, 4451-4458 (1994).

31. Tang, C. Y., Bezanilla, F. \& Papazian, D. M. Extracellular $\mathrm{Mg}^{2+}$ modulates slow gating transitions and the opening of Drosophila ether-a-go-go potassium channels. J. Gen. Physiol. 115, 319-337 (2000).

32. García-Ferreiro, R. E. et al. Mechanism of block of hEagl $\mathrm{K}^{+}$Channels by imipramine and astemizole. J. Gen. Physiol. 124, 301-317 (2004).

33. Gomez-Varela, D. et al. Different relevance of inactivation and F468 residue in the mechanisms of hEag1 channel blockage by astemizole, imipramine and dofetilide. FEBS Lett. 580, 5059-5066 (2006).

34. Downie, B. R. et al. Eag1 expression interferes with hypoxia homeostasis and induces angiogenesis in tumors. J. Biol. Chem. 283, 36234-36240 (2008).

35. Alonso-Ron, C., de la Pena, P., Miranda, P., Dominguez, P. \& Barros, F. Thermodynamic and kinetic properties of amino-terminal and S4-S5 loop HERG channel mutants under steady-state conditions. Biophys. J. 94, 3893-3911 (2008)

36. Wang, S., Liu, S., Morales, M. J., Strauss, H. C. \& Rasmuson, R. L. A quantitaive analysis of the activation and inactivation kinetics of HERG expressed in Xenopus oocytes. J. Physiol. 502, 45-60 (1997).

37. Oinuma, H. et al. 4'-[(4-Piperidyl)carbonyl]methanesulfonanilides as potent, selective, bioavailable class III antiarrhythmic agents. J. Med. Chem. 33, 903-905 (1990).

38. Ishii, K., Nagai, M., Takahashi, M. \& Endoh, M. Dissociation of E-4031 from the HERG channel caused by mutations of an amino acid results in greater block at high stimulation frequency. Cardiovasc. Res. 57, 651-659 (2003).

39. Zhou, Z., Gong, Q., Epstein, M. L. \& January, C. T. HERG channel dysfunction in human long QT syndrome. Intracellular transport and functional defects. J. Biol. Chem. 273, 21061-21066 (1998).

40. Es-Salah-Lamoureux, Z., Xiong, P. Y., Goodchild, S. J., Ahern, C. A. \& Fedida, D. Blockade of permeation by potassium but normal gating of the G628S nonconducting hERG channel mutant. Biophys. J. 101, 662-670 (2011).

41. Viloria, C. G., Barros, F., Giraldez, T., Gomez-Varela, D. \& de la Peña, P. Differential effects of amino-terminal distal and proximal domains in the regulation of human erg $\mathrm{K}^{+}$channel gating. Biophys. J. 79, 231-246 (2000).

42. Fowler, P. W. \& Sansom, M. S. P. The pore of voltage-gated potassium ion channels is strained when closed. Nat. Commun. 4, 1872 (2013).

43. Yifrach, O. \& MacKinnon, R. Energetics of pore opening in a voltage-gated $\mathrm{K}^{+}$ channel. Cell 111, 231-239 (2002).

44. Hardman, R. M., Stansfeld, P. J., Dalibalta, S., Sutcliffe, M. J. \& Mitcheson, J. S. Activation gating of hERG potassium channels: S6 glycines are not required as gating hinges. J. Biol. Chem. 282, 31972-31981 (2007).

45. Choveau, F. S. et al. Opposite effects of the S4-S5 linker and PIP(2) on voltagegated channel function: KCNQ1/KCNE1 and other channels. Front. Pharmacol. 3, 125 (2012)

46. Lu, Z., Klem, A. M. \& Ramu, Y. Ion conduction pore is conserved among potassium channels. Nature 413, 809-813 (2001).
47. Ledwell, J. L. \& Aldrich, R. W. Mutations in the S4 region isolate the final voltage-dependent cooperative step in potassium channel activation. J. Gen. Physiol. 113, 389-414 (1999).

48. Pathak, M., Kurtz, L., Tombola, F. \& Isacoff, E. The cooperative voltage sensor motion that gates a potassium channel. J. Gen. Physiol. 125, 57-69 (2005).

49. Soler-Llavina, G. J., Chang, T.-H. \& Swartz, K. J. Functional interactions at the interface between voltage-sensing and pore domains in the Shaker Kv channel. Neuron 52, 623-634 (2006).

50. Wang, J., Trudeau, M. C., Zappia, A. M. \& Robertson, G. A. Regulation of deactivation by an amino terminal domain in human ether-a-go-go-related gene potassium channels. J. Gen. Physiol. 112, 637-647 (1998).

51. Fernandez-Trillo, J. et al. Molecular determinants of interactions between the $\mathrm{N}$-terminal domain and the transmembrane core that modulate hERG $\mathrm{K}^{+}$ channel gating. PLoS ONE 6, e24674 (2011).

52. Chen, J., Zou, A., Splawski, I., Keating, M. T. \& Sanguinetti, M. C. Long QT syndrome-associated mutations in the Per-Arnt-Sim (PAS) domain of HERG potassium channels accelerate channel deactivation. J. Biol. Chem. 274, 10113-10118 (1999).

53. Alonso-Ron, C. et al. Participation of HERG channel cytoplasmic structures on regulation by the G protein-coupled TRH receptor. Pflugers Arch. 457, 1237-1252 (2009).

54. Phartiyal, P., Jones, E. M. C. \& Robertson, G. A. Heteromeric assembly of human ether-à-go-go-related gene (hERG) 1a/1b channels occurs cotranslationally via N-terminal interactions. J. Biol. Chem. 282, 9874-9882 (2007).

55. Jenke, M. et al. C-terminal domains implicated in the functional surface expression of potassium channels. EMBO J. 22, 395-403 (2003).

56. Schwake, M. et al. Structural determinants of M-type KCNQ (Kv7) K ${ }^{+}$ channel assembly. J. Neurosci. 26, 3757-3766 (2006).

57. Villmann, C., Strutz, N., Morth, T. \& Hollmann, M. Investigation by ion channel domain transplantation of rat glutamate receptor subunits, orphan receptors and a putative NMDA receptor subunit. Eur. J. Neurosci. 11, 1765-1778 (1999).

58. Gomez-Posada, J. C. et al. Kv7 channels can function without constitutive calmodulin tethering. PLoS ONE 6, e25508 (2011).

59. Chen, Y. et al. Functional Kv10.1 Channels localize to the inner nuclear membrane. PLoS ONE 6, e19257 (2011).

\section{Acknowledgements}

We thank K. Dümke and A. Sporning for expert technical assistance. J.C.G.-P. was partially financed by the Department of Education, Universities and Investigation of the Basque Government within the programme of perfection of doctors abroad. Supported by the Max-Planck Society, the Spanish Ministry of Science and Innovation (MICINN) Consolider-Ingenio programme (Grant CSD2008-00005) and the 2013 Principado of Asturias Government grant SV-PA-13-ECOEMP-69.

\section{Author contributions}

E.L., J.C.G.-P., P.d.l.P. and A.P.T. generated the constructs. E.L., J.C.G.-P., A.P.T., J.F.-T., U.L., F.B. and L.A.P. collected the data. E.L., J.C.G.-P., P.d.l.P., W.S., F.B. and L.A.P. designed the study. F.B., P.d.l.P. and L.A.P. wrote the manuscript, all authors discussed the results and commented on the manuscript.

\section{Additional information}

Supplementary Information accompanies this paper at http://www.nature.com/ naturecommunications

Competing financial interests: The authors declare no competing financial interest.

Reprints and permission information is available online at http://npg.nature.com/ reprintsandpermissions/

How to cite this article: Lörinczi, É. et al. Voltage-dependent gating of $\mathrm{KCNH}$ potassium channels lacking a covalent link between voltage-sensing and pore domains. Nat. Commun. 6:6672 doi: 10.1038/ncomms7672 (2015).



This work is licensed under a Creative Commons Attribution 4.0 International License. The images or other third party material in this article are included in the article's Creative Commons license, unless indicated otherwise in the credit line; if the material is not included under the Creative Commons license, users will need to obtain permission from the license holder to reproduce the material. To view a copy of this license, visit http://creativecommons.org/licenses/by/4.0/ 